\title{
Tri-trophic interactions of a predator- parasite-host assemblage in New Zealand
}

BY

\section{KIRSTY JANE YULE}

\author{
A thesis submitted to \\ Victoria University of Wellington \\ in fulfilment of the requirements for the degree of \\ Doctor of Philosophy
}

Victoria University of Wellington

(2016)

TE WHARE WĀNANGA O TE ŪPOKO O TE IKA A MĀUI

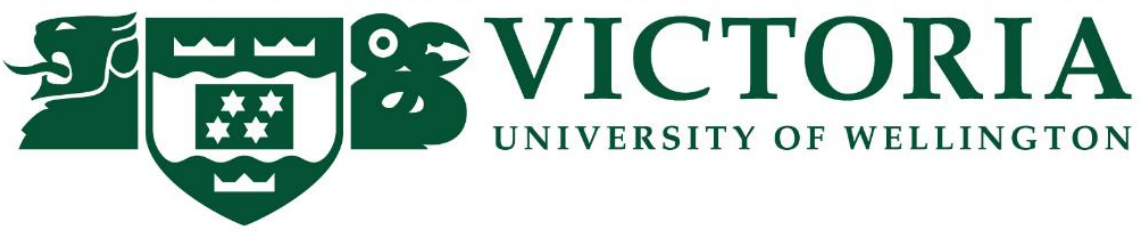


This thesis was conducted under the supervision of

Associate Professor Kevin Burns (Primary Supervisor)

Victoria University of Wellington, New Zealand 


\section{Abstract}

Parasites are ubiquitous and the antagonistic relationships between parasites and their hosts shape populations and ecosystems. However, our understanding of complex parasitic interactions is lacking. New Zealand's largest endemic moth, Aenetus virescens (Lepidoptera: Hepialidae) is a long-lived arboreal parasite. Larvae grow to $100 \mathrm{~mm}$, living $\sim 6$ years in solitary tunnels in host trees. Larvae cover their tunnel entrance with silk and frass webbing, behind which they feed on host tree phloem. Webbing looks much like the tree background, potentially concealing larvae from predatory parrots who consume larvae by tearing wood from trees. Yet, the ecological and evolutionary relationships between the host tree, the parasitic larvae, and the avian predator remain unresolved.

In this thesis, I use a system-based approach to investigate complex parasite-host interactions using $A$. virescens (hereafter "larvae") as a model system. First, I investigate the mechanisms driving intraspecific parasite aggregation (Chapter 2). Overall, many hosts had few parasites and few hosts had many, with larvae consistently more abundant in larger hosts. I found no evidence for densitydependent competition as infrapopulation size had no effect on long-term larval growth.

Host specificity, the number of species utilised from the larger pool available, reflects parasite niche breadth, risk of extinction and ability to colonise new locations. In Chapter 3, I investigate larvae host specificity in relation to host nutritional rewards (phloem turnover and phloem sugar content) and host defences (bark thickness and wood density). The number of species parasitized was not explained by tree abundance, nutritional rewards or wood density. However, the number of trees parasitised declined significantly with increasing bark thickness indicating host external defences are an important driver of host specificity.

Camouflage in animals has traditionally been considered an anti-predator adaptation. Yet the adaptive consequences of camouflage, i.e. increased survivability via predator avoidance, has rarely been tested. In Chapter 4 , I show that larvae webbing is visually cryptic to predating kaka, yet did not protect larvae from 
attack. Instead, cryptic webbing aids larvae thermoregulation suggesting crypsis is non-adaptive. These results support an exciting newly emerging paradigm shift that indicates the evolution of camouflage in animals may be more to do with abiotic conditions than biotic signalling.

Males are often the "sicker sex", experiencing higher pathogen and parasite loads than females. In Chapter 5, I construct the largest host-parasite database to date, spanning 70 animal and 22 plant families, from which I conduct a meta-analysis testing for male biased susceptibility (MBS). Then, I develop a theoretical model that explain MBS as a result of parasite-offspring competition for female resources. I present the first, unified model that explains male-biased susceptibility in animals and plants and provide parameters for model replication, applicable to almost all host-parasite pairings on Earth.

This thesis presents the first investigations of the natural history of Aenetus virescens larvae, their relationships with host trees, and the interactions with their avian predator. The results herein support existing theories of parasite aggregation and host specificity from a novel perspective. Furthermore, results support a newly emerging paradigm shift in animal camouflage evolution, and suggest a unified explanation for male biased susceptibility in animals and plants. The results herein help further our understanding of complex antagonistic relationships between parasites and their hosts, presenting novel theories on which future research can be built. 


\section{Acknowledgements}

This thesis would not have been possible without my primary supervisor, KC Burns, who gave me the freedom to walk the path of a scientist, allowed me to explore my own interests and discover my own direction, whilst training me to always listen to what mother nature is telling me. I am forever grateful for KC's encouragement and support, and for always pushing me to be a better scientist.

Thank you to the Burns lab group Amanda, Rachael, Patrick, Julia, Latu, Bart, and Matt for our regular "lab meetings", our endless tea and catch ups and for the encouragement and support during this research. Thank you to my officemates Jen, Ashley, Emily and Katie for allowing me to distract them with questions and general musings.

To all of the research assistants who gave up their spare time to help with data collection, even on days when it was hailing, thank you! Rosi, Jake, Caitlin, Hannah, Nurul, Annemieke, Leah, Sean, and Clara - Your efforts and company made hard work easier, faster and infinitely more enjoyable. In particular, thank you to Brie for countless days of patiently hunting caterpillars, for incredible organisational skills that kept us on track and for always being cheerful and happy to help, you are a star! A massive thank you to Mary, Adam, and Leah for helping to sort through the large stack of manuscripts that were used in Chapter 5 . I am sure I would still be working on them had I gone it alone!

To the ladies in KK507 - you have saved the day so many times and made the mornings so much more cheerful, thank you for the work you do!

The past four years would have been a very different journey had it not been for the love and support of my family and friends. Thank you to Meghan Cooling for always being willing to talk caterpillars and being an invaluable sounding board, as well as stepping in at the last minute when needed. Thank you to Grace and Amanda for encouragement and support, and wonderful nights of bubbles and dancing that made this whole process infinitely more human. A massive thank you to Jenah Shaw, without whom the final state of this thesis would have been considerably more flawed, and to both Jenah and James for being so supportive and throwing the best kitchen dance parties! 
Thank you very much to Christine; our regular debriefs, our many outings and adventures, and the countless days of upcycling, crafting and op-shopping have been a wonderful sidestep from the intensities of research. I am grateful that you always understood what I was trying to say, even when I wasn't making sense! The Conway's are my adopted NZ family and I will be forever grateful for being so warmly included and for always feeling like one of the family.

The whole process of being so far from home whilst undertaking this research was made possible through the support of friends across the miles. I am eternally thankful to Donna \& Matt, Fiona \& Phill, Angela \& Mike, Katie \& Grace, Conor, Gemma, and Laura. Thank you for keeping in touch and going the extra mile to make the time difference work, and to visit when possible. I am thankful for your friendships and support, your enthusiasm for my adventure, and for always being available on my scarce trips home. In particular, thank you to Ham and Fifi for incredible editing skills and advice on manuscripts - ladies, you are awesome! My family deserve a special thanks for the massive amount of love, support and encouragement they have shown throughout this experience. I am always grateful for the messages, letters, photographs and emails that get sent my way, and for always making it a joy to come home. In particular, I am thankful to both of my Grans' - they shaped my life significantly with their passion, strength and commitment to family.

I am so grateful to my amazing wee brother Michael for always being a pillar of strength and support, a source of laughter and fun, and for his unwavering belief in my abilities. Plus, the stories and pictures of Jack were always a delight to receive!

My biggest thanks go to my Mumsy for all the years of unwavering support, encouragement and excitement for the weird and wonderful things I have been lucky enough to do in my career. I am eternally grateful for the love and strength you give without question, and for instilling in me a belief that I can do anything. Thank you for being almost as excited as I was when I got the chance to follow this dream, even when it meant me moving to the opposite side of the planet. For being my rock, my support, and my best friend, I am immensely grateful and none of this would have been possible without you. 


\section{Contents}

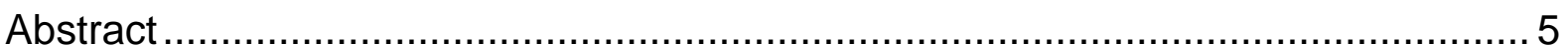

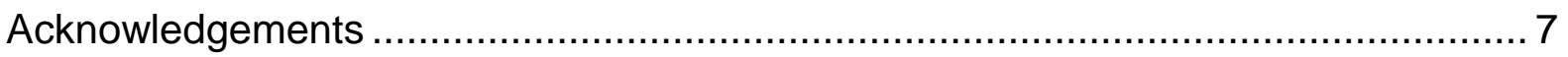

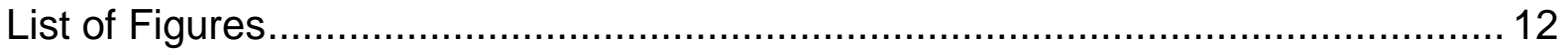

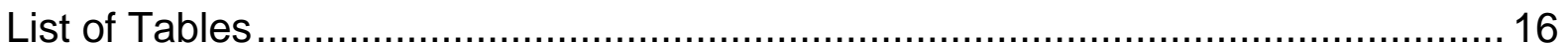

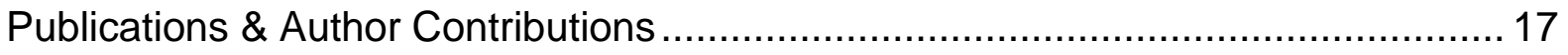

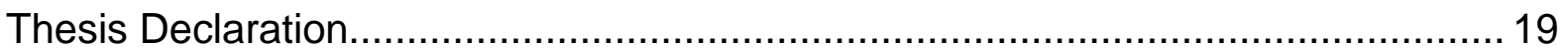

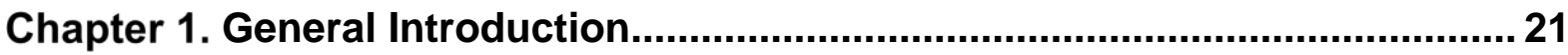

1.1. The Role of Parasites in Ecology and Evolution ......................................... 23

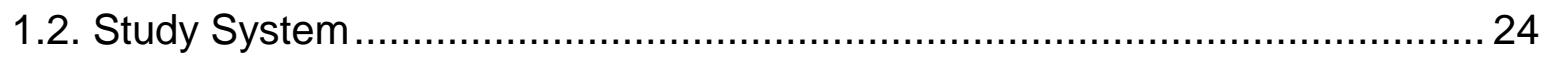

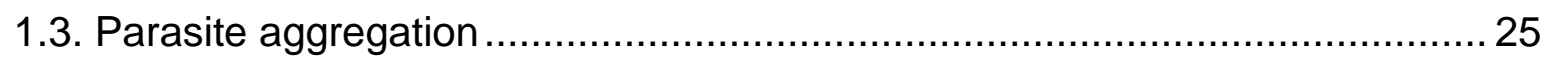

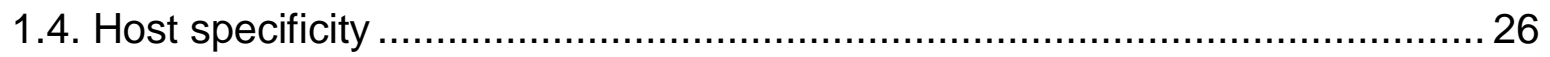

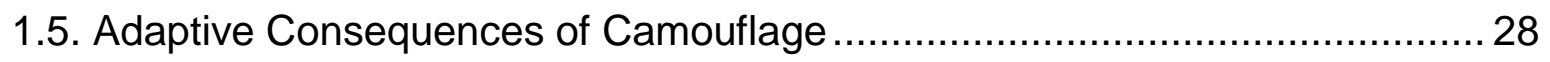

1.6. Male-biased Host Susceptibility ........................................................... 29

1.7. Research Aims and Thesis Overview ...................................................... 31

Chapter 2. Drivers of aggregation in a novel arboreal parasite: the influence of

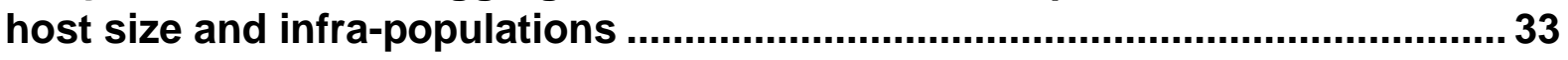

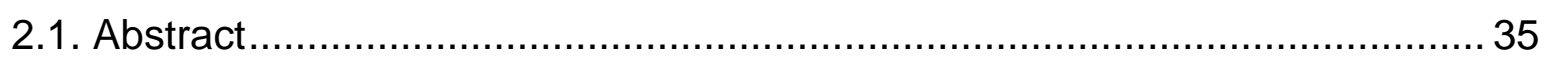

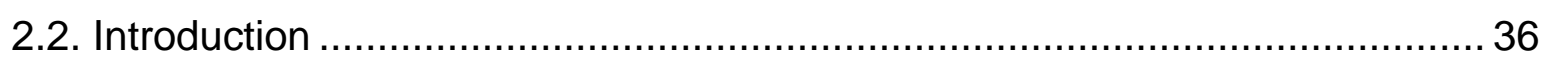

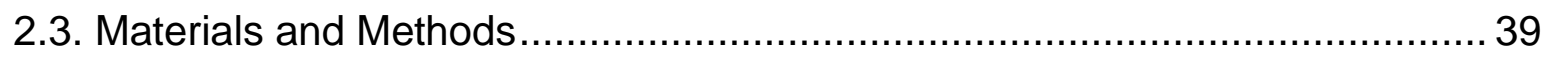

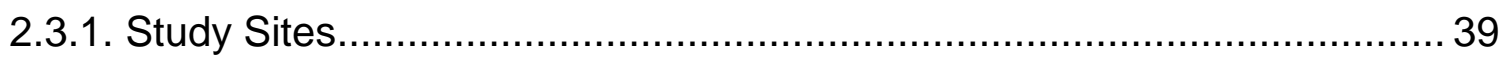

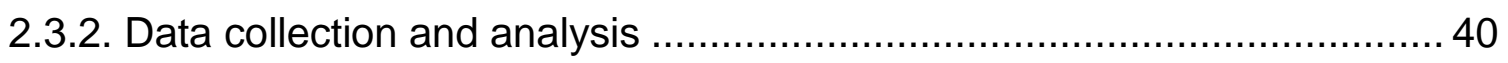

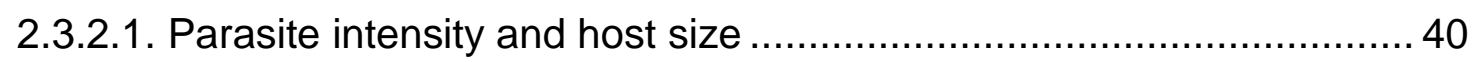

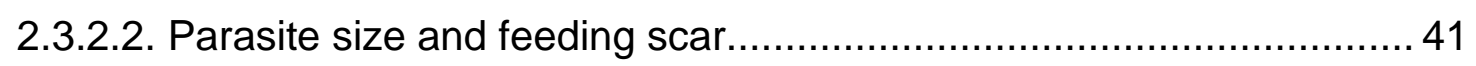

2.3.2.3. Parasite growth and intensity ...................................................... 41

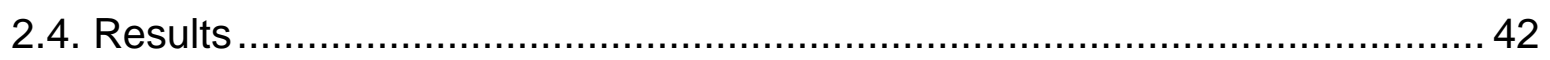

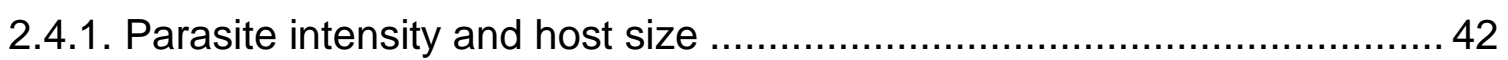

2.4.2. Parasite size and feeding scar ......................................................... 44

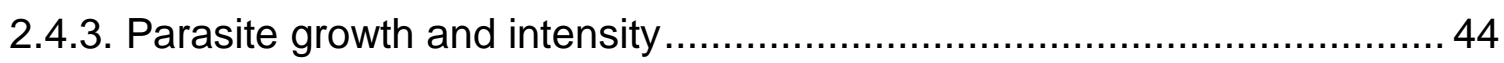

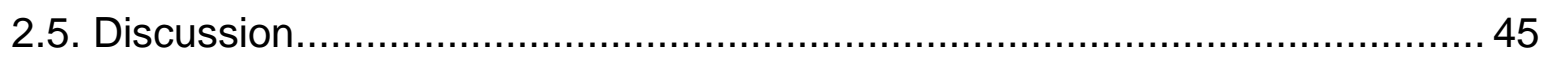

Chapter 3. Host defensive traits explain parasite host specificity in multiple

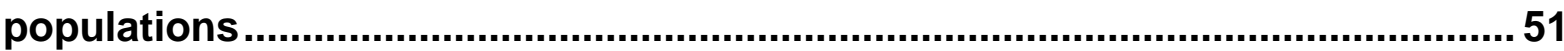

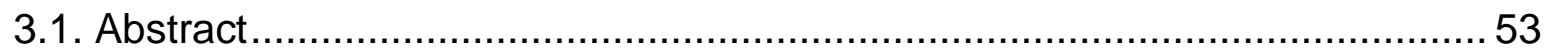

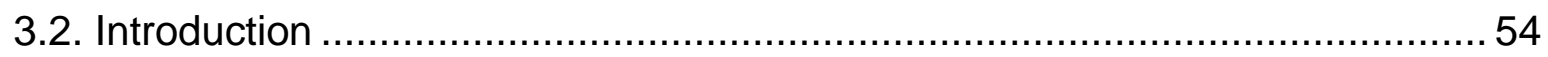

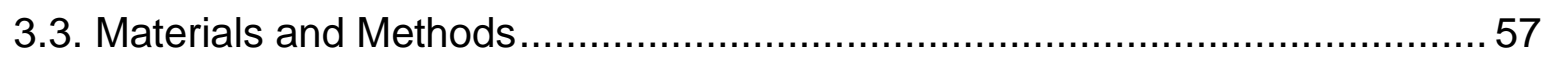




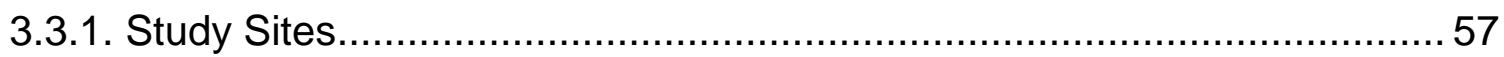

3.3.2. Does forest composition differ between sites? ..................................... 58

3.3.3. Does tree abundance predict number of trees parasitised?.................... 59

3.3.4. Do tree traits determine number of trees parasitised? ........................... 60

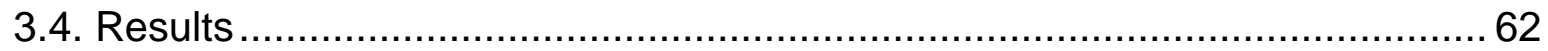

3.4.1. Does forest composition differ between sites?..................................... 62

3.4.2. Does tree abundance predict number of trees parasitised?..................... 62

3.4.3. Do tree traits determine the number of trees parasitised? ...................... 67

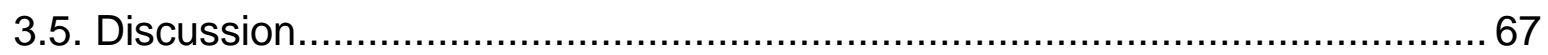

Chapter 4. Non-adaptive camouflage: crypsis can obscure adaptive

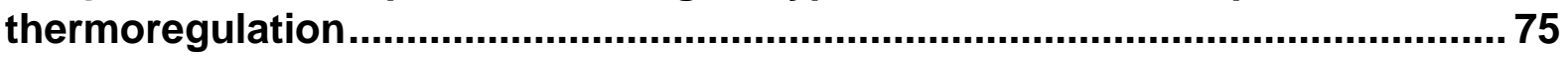

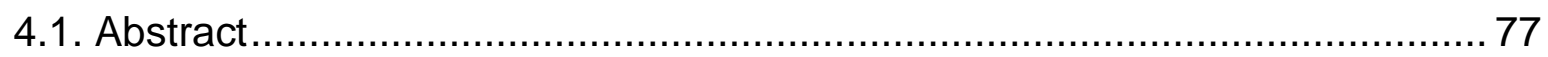

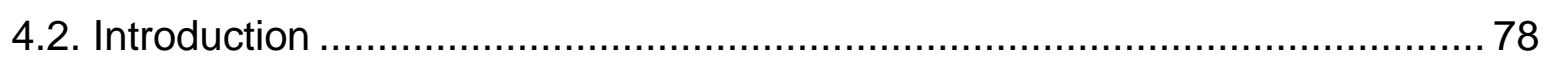

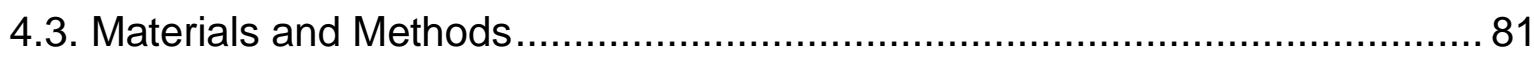

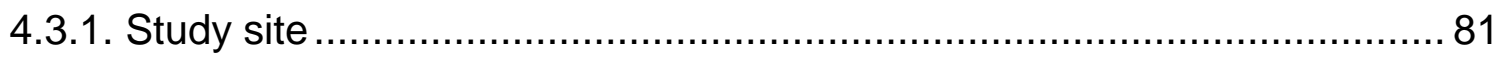

4.3.2. Experiment 1: Is webbing visually cryptic to parrots? ............................. 81

4.3.3. Experiment 2: Does cryptic webbing protect larvae from parrot attack? .. 83

4.3.4. Experiment 3: Does webbing increase temperature inside larvae tunnels?

4.3.5. Experiment 4: Does changes in temperature brought about by webbing affect larval growth rates? ..................................................................... 84

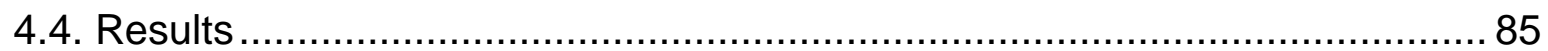

4.4.1. Experiment 1: Is webbing visually cryptic to parrots? ............................. 85

4.4.2. Experiment 2: Does cryptic webbing protect larvae from parrots attack? 86

4.4.3. Experiment 3: Does webbing increase temperature inside larvae tunnels?

4.4.4. Does changes in temperature brought about by webbing affect larval

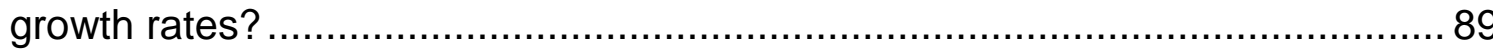

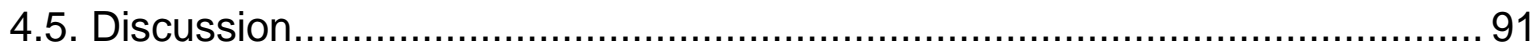

Chapter 5. A unified explanation for male-biased susceptibility in animals and

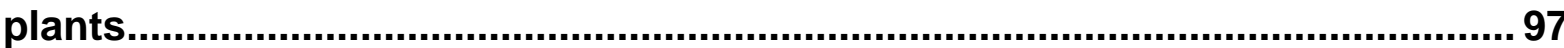

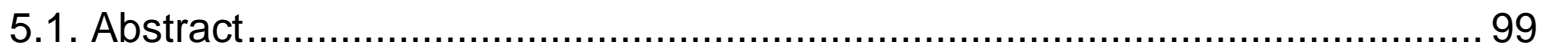

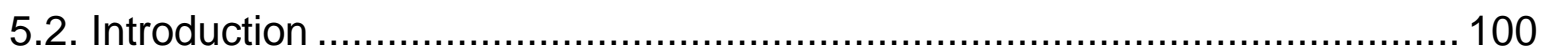

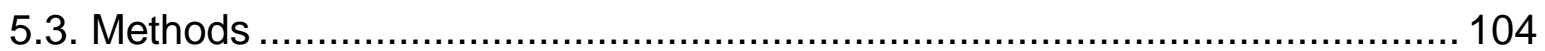

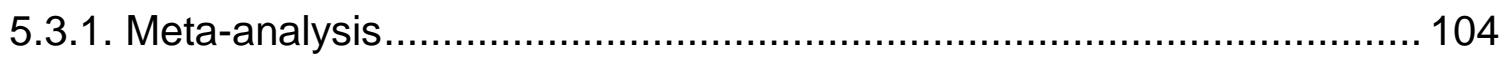

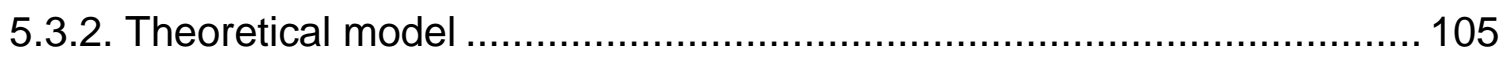




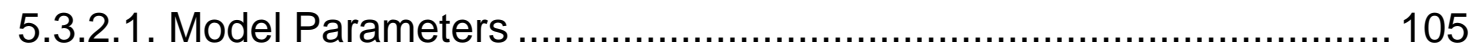

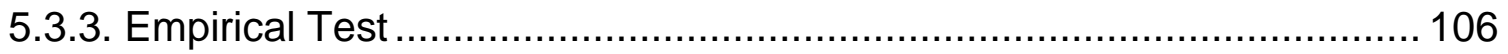

5.3.4. Testing the alternative explanation for MBS ……............................ 110

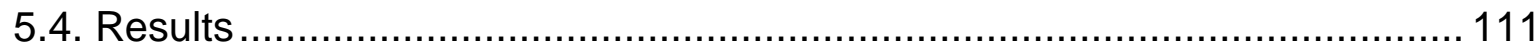

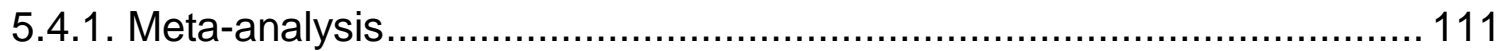

5.4.2. Empirically testing the theoretical model.......................................... 112

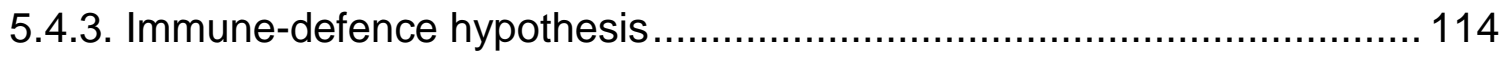

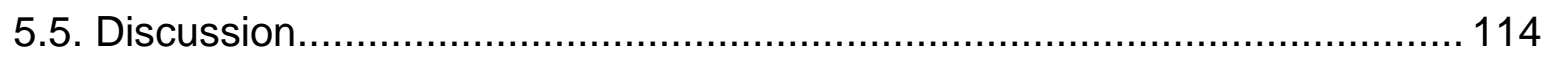

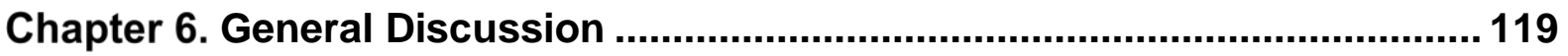

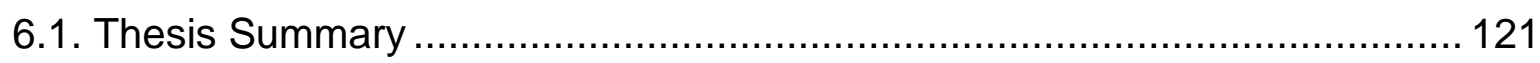

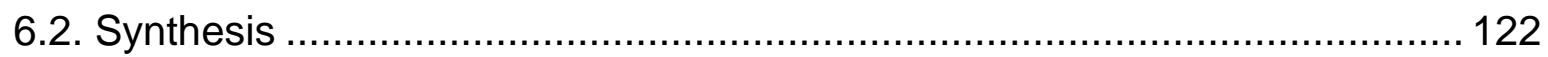

6.2.1. Intraspecific parasite aggregation on hosts...................................... 122

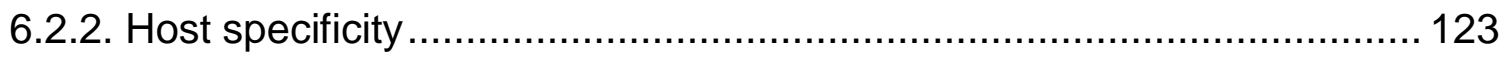

6.2.3. Adaptive consequences of camouflage ………................................ 125

6.2.4. Male-biased host susceptibility ................................................... 126

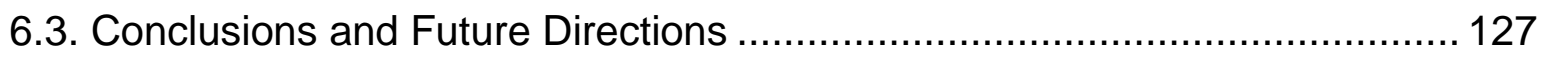

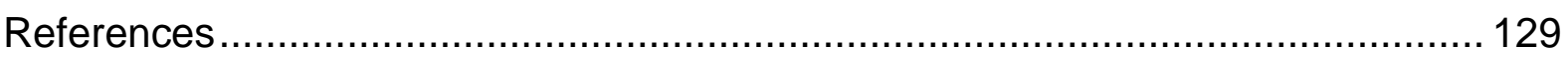

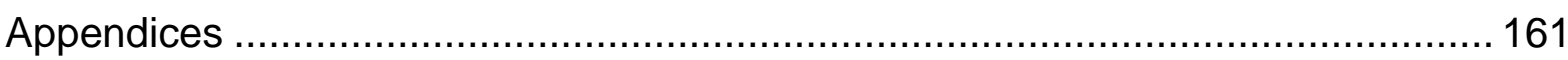

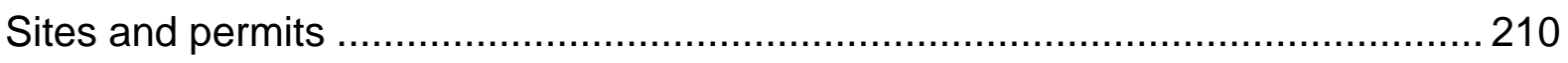

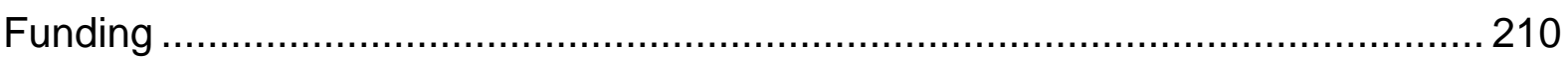




\section{List of Figures}

Figure 2.2.1 New Zealand's largest endemic moth, Aenetus virescens. (a) Adult moth (female), (b) parasitic arboreal-phase larva, and (c) parasite feeding scar on Aristotelia serrata host tree, covered with silk and frass webbing (webbing is outlined). Error! Bookmark not defined.

Figure 2.4.1 Influence of Aristotelia serrata host tree size on arboreal parasite Aenetus virescens infra-population. Error! Bookmark not defined.

Figure 2.4.2. Growth of arboreal parasite Aenetus virescens feeding scar (proxy for parasite body size) between year 1, February 2013 (feeding scar , FA2013) and year 2, February 2014 (FA2014). Dashed line shows isometry (1:1). Solid line shows reduced major axis (RMA) Error! Bookmark not defined.

Figure 2.4.3. Influence of infra-population on Aenetus virescens arboreal parasite growth on (a) the sum of conspecific larvae in an Aristotelia serrata host tree, (b) the sum of conspecific larvae occurring above a focal individual in a host tree (upstream competitors), (c) the summed size of all feeding scars with larvae present in a host tree, and (d) the summed size of feeding scars with larvae present, occurring above the focal individual in a host tree. Error! Bookmark not defined.

Figure 3.2.1. (a) Aenetus virescens larvae inside a host sapling, (b) Webbing covering the tunnel entrance of $A$. virescens, (c) Feeding scar surrounding the tunnel entrance after webbing was removed, (d) the North Island Kaka, Nestor meridionalis septentrionalis, (e) Damage to host trees after kaka excavated an $A$. virescens larvae, (f) Adult female A. virescens. 55

Figure 3.3.1. Wellington Region of New Zealand's North Island. Site 1: Butterfly Creek, East Harbour Regional Park; Site 2: Kaitoke Regional Park, Pakuratahi; and Site 3: Zealandia, Karori. 58

Figure 3.4.1. Y1: Total number of trees with parasites in ranked order of decreasing abundance, and Y2: Total number of trees. (a) Site 1: Butterfly Creek ( $\mathrm{n}=30$ tree species). (b) Site 2: Kaitoke Regional Park ( $n=24$ tree species). (c) Site 3: 
Zealandia ( $n=36$ tree species). X-axis provides the number assigned to each tree species as listed in Table 3.1.

Figure 3.5.1. Influence of tree traits on host specificity of Aenetus virescens larvae for the 24 most common tree species. (a) Phloem turnover (CER - khoms), (b) Phloem sugar content ('Brix), (c) Wood Density (g-cm3), (d) Bark Thickness (mm). All Y-axes show the residuals of the number of trees parasitised when the other three traits and tree size $(\mathrm{DBH})$ are accounted for. The numbered points represent the numbers assigned to each tree species specified in Table 3.1.

Figure 4.2.1. (a) Adult female Aenetus virescens, New Zealand's largest endemic moth. (b) A. virescens larvae in its self-excavated "7"-shaped tunnel inside a host sapling. (c) Feeding scar with webbing freshly removed where $A$. virescens nocturnally extract phloem; larvae can be seen emerging from the tunnel entrance after the authors flooded the tunnel. (d) Webbing made from silk, frass and debris from the tree bark covers tunnel entrance. (e) Nestor meridionalis are the main predator of $A$. virescens and use powerful beaks to excavate bark and wood from trees to consume larvae. (f) The damage incurred by host trees after $N$. meridionalis excavate wood to consume $A$. virescens larvae. All scale bars represent $1 \mathrm{~cm}$. 80

Figure 4.4.1. Mean ( \pm SE, shaded areas) reflectance spectra for removed (a), new (c), and old (e) Aenetus virescens webbing (red) and the background bark surrounding webbing on host trees (blue). Volume taken up in avian colour space between removed (b), new (d) and old (f) A. virescens webbing (red) and background bark (blue). Grey regions indicate an overlap under average UV and forest shade light conditions, with overlap \% and mean just noticeable differences (JND) for chromatic (dS) and achromatic (dL) \pm standard error detailed below each figure. Inserts in (b), (d) and (f) show bark and webbing as viewed in a projection plot of a tetrahedral avian colour space under average UV and forest-shade light conditions.

Figure 4.4.2. Survival of Aenetus virescens larvae over a 26-month field experiment in which the background colour surrounding their cryptic webbing was manipulated to create increased conspicuousness. Time until discovery and predation risk were 
statistically the same for all treatments, indicating cryptic webbing did not protect larvae from predation.

Figure 4.4.3. Effect of webbing (removed or present) and sampling position (Front: within $1 \mathrm{~cm}$ of webbing; Back: furthest point into tunnel, between 4 and $8 \mathrm{~cm}$ ) on temperature within Aenetus virescens larval tunnels. Red solid line represents the mean ambient outside temperature (within $5 \mathrm{~cm}$ of tree surface) for all sampling days. Contrasts were analysed using the HSD Tukey test after a nested ANOVA -all treatments were significantly different from each other $(P<0.001)$ and tunnels with webbing were significantly higher in temperature than tunnels with webbing removed $(P<0.001)$ 90

Figure 4.4.4. Relationship between the average tunnel temperature and the average annual growth of $A$. virescens larvae.

Figure 5.3.1. The relationship between the number of parasites on male hosts $\left(P_{3}\right)$ and tree diameter at breast height $(D=1.25 \mathrm{~m})$ (blue), and the number of parasites on female hosts $\left(P_{\varphi}\right)$ and $D($ red).

Figure 5.3.2. The relationship between (a) the number of seeds (S) and tree DBH (D) for unparasitised female trees, and (b) the number of parasites (P) and tree DBH (D) for male trees (no seeds). The equation in each figure shows the slope $(\mathrm{m})$ and intercept (b) for each relationship used to solve for $D$.

Figure 5.4.1. Frequency of expected bias from 1000 random simulations for: (a) animal families $(n=70)$ and (b) plant families $(n=22)$. " 0 " indicates an entirely female bias and " 1 " indicates an entirely male bias. Dashed lines indicate the mean observed bias.

Figure 5.4.2. Relationship between the number of parasites on female trees $\left(P_{\varphi}\right)$ plus the coefficient equating the energetic cost of seeds to the energetic cost of parasites $\left(\propto_{N S} S\right.$, ) and the number of parasites on male trees $\left(\mathrm{P}_{\supset}\right)$ (Slope: 1.06, lower $\mathrm{Cl}=0.90$, upper $\mathrm{Cl}=1.21$ ). Solid line is line of best fit from linear regression; dashed line is the line of isometry $(1: 1)$ 113 
Figure 5.4.3. Difference between bark thickness on female (red) and male (blue) Aristotelia serrata host trees.

Figure A.1. Correlation matrix of Aenetus virescens body components - weight, head width, tail width, length and volume - used to deduce the allometric relationships between larval body parts. $r^{2}$ values are shown in corresponding boxes in the bottom

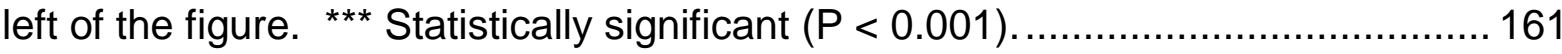




\section{List of Tables}

Table 3.1. Tree nomenclature, abundance, and prevalence of parasitic Aenetus virescens for all tree species $(n=49)$ recorded in the Wellington Region of New Zealand's North Island. Site 1: Butterfly Creek, East Harbour Regional Park; Site 2: Kaitoke Regional Park; Site 2: Kaitoke Regional Park, Pakuratahi; Site 3: Zealandia, Karori. (g) in "Endemic" column denotes endemic genus as well as species. 63

Table A.1. Plant host - parasite pairings used in meta-analysis in chapter 5. Column one lists host plant order, family, and genus \& species in alphabetical order. Parasites are listed to order, species names where known. Bias in susceptibility is either male $(M)$ or female $(F)$, whether parasite prevalence $(P)$ or intensity $(I)$ was reported by the authors. Sample size refers to the number of hosts inspected, reported where known; any sample sizes that occur in the male column only relates to both male and female sample sizes combined where authors did not specific by gender specifically. 163

Table A.2. Animal host - parasite pairings used in meta-analysis in chapter 5. Column one lists host animal order, family, genus and species in alphabetical order. Parasites are listed to order, species names where known. Bias in susceptibility is either male $(M)$ or female $(F)$, whether parasite prevalence $(P)$ or intensity $(I)$ was reported by the authors. Sample size refers to the number of hosts inspected, reported where known; any sample sizes that occur in the male column only relates to both male and female sample sizes combined where authors did not specific by gender specifically. 169 


\section{Publications \& Author Contributions}

\section{Published}

Yule KJ, Burns KC (2014) Drivers of aggregation in a novel arboreal parasite: the influence of host size and infra-populations. Int J Parasitol 45:197-202. doi: 10.1016/j.jpara.2014.10.007

Author contributions ${ }^{1}$ : KJY conceived the idea, developed hypotheses and methods, collected data, performed statistical analysis, wrote the manuscript and submitted it for publication. KCB advised on statistical analysis, and revisions to manuscript.

\section{$\underline{\text { In review }}$}

Yule, KJ \& Burns, KC (revised \& resubmitted) Host defensive traits explain parasite host specificity in multiple populations. Evolutionary Ecology.

Author contributions ${ }^{1}$ : KJY \& KCB conceived the idea, KJY developed hypotheses methods, collected data, performed statistical analysis, wrote the manuscript and submitted it for publication. KCB advised on statistical analysis, and revisions to manuscript.

Yule, KJ \& Burns, KC (In review) Non-adaptive crypsis: Camouflage obscures adaptive thermoregulation. Functional Ecology.

Author contributions $^{1}$ : KJY \& KCB conceived the idea, KJY developed hypotheses and methods, collected data, performed statistical analysis, wrote the manuscript and submitted it for publication. KCB helped develop concepts, advised on statistical analysis, and revisions to manuscript.

\section{In preperation}

Yule, KJ \& Burns, KC (In Preperation) A unified explanation for male biased susceptibility in animals and plants.

Author contributions ${ }^{1}$ : KJY \& KCB conceived the idea, KJY developed methods, collected data, performed statistical analysis, wrote the manuscript and submitted it for publication. KCB helped develop concepts, models and statistical analysis.

${ }^{1}$ Authors initials: Kirsty Yule (KJY). Kevin Burns (KCB) 


\section{Cover Photographs}

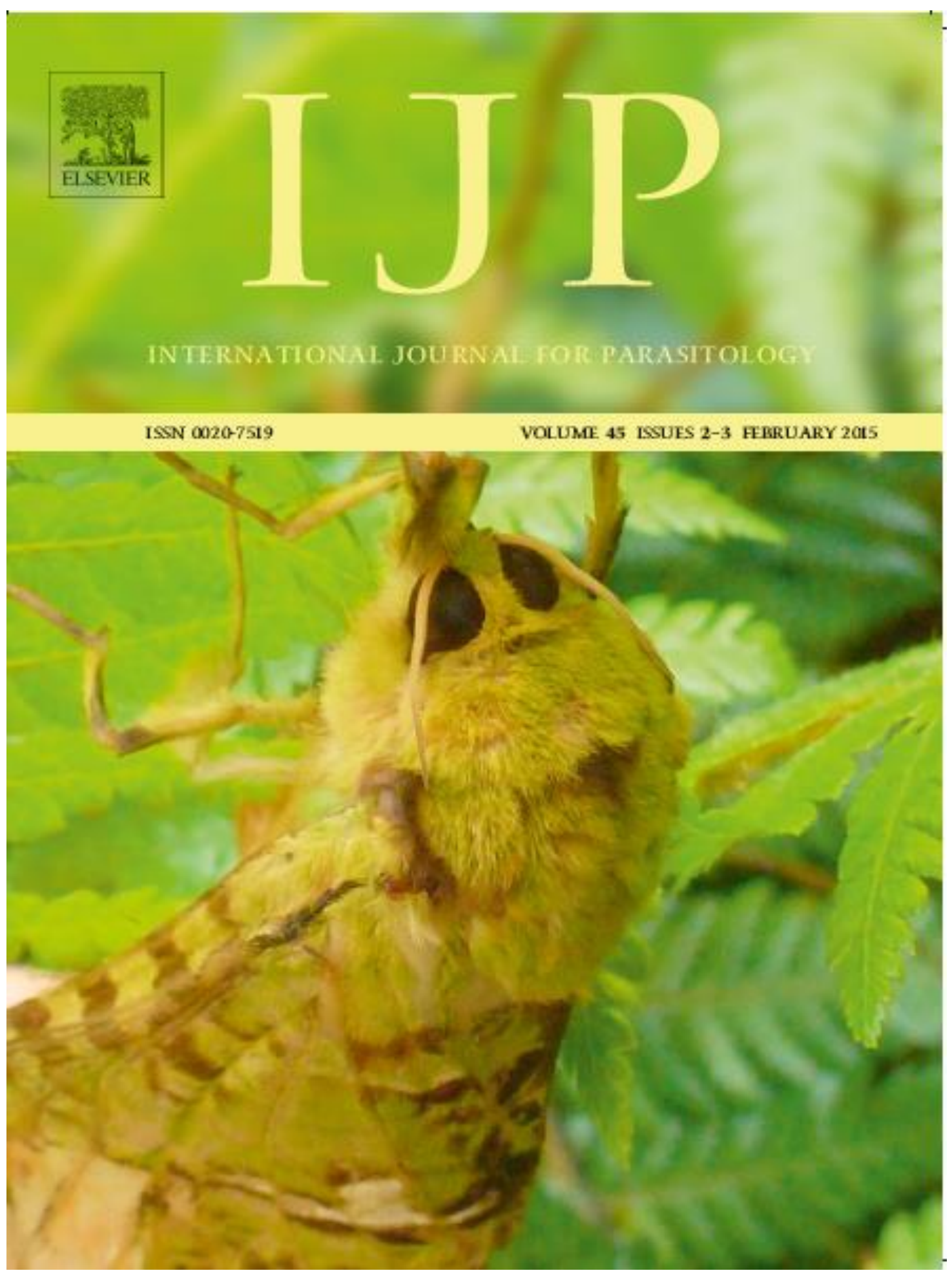

Cover photo: Yule, KJ, International Journal for Parasitology, February 2015. 


\section{Thesis Declaration}

I hereby declare that this thesis is my own work and that all sources quoted, paraphrased or otherwise referred to, have been acknowledged in the references at the end of this document. To the best of my knowledge, this thesis neither contains material previously published or written by another person, nor material which to a substantial extent has been accepted for the award of any other degree or diploma of the university or other institutes of higher learning, except where due to acknowledgement it has been made clear in the text.

Chapters 2, 3, 4, and 5 have been written individual research papers, so there is inevitable repetition between the introductions and discussions of these chapters. All of these chapters contain contributions from my primary supervisor, Kevin Burns, (See Author Contributions) however all chapters are written here in first person singular. 


\section{Chapter 1. General Introduction}




\subsection{The Role of Parasites in Ecology and Evolution}

Parasites are ubiquitous and represent the most common life strategy on Earth (Lafferty et al. 2006; Dobson et al. 2008). Once the focus solely of parasitologists concerned with taxonomic diversity, life cycles and pathology, a growing body of evidence now suggests that parasites have far-reaching consequences for ecology, evolution, disease transmission, invasive species management, human health and conservation, increasing the focus on parasites as a significant driving force in nature (Zuk \& Stoehr 2002). Parasites represent the majority of species diversity on Earth (Nichols \& Gómez 2011). Rohde (1993) suggested $>40 \%$ of all species are parasitic. The number of parasitic helminth species, for example, are estimated to exceed the number of their vertebrate hosts by at least 50\% (Poulin \& Morand 2004). From microscopic viruses and bacteria to endoparasitic protozoans and helminths, to ectoparasitic ticks and plants, parasites form a formidable and diverse group of organisms that rely solely on their hosts for survival. Yet the complex interactions between parasites and their hosts often limit the generalisations that can be made regarding specific host or parasite species (Poulin \& Forbes 2011). Thus, understanding of the complex drivers and outcomes of parasitic relationships remains lacking.

Evolution through natural selection determines the niche and ecological interactions of organisms across the globe (Darwin 1858; Wallace 1870). No organism lives in isolation, but rather is interconnected to numerous others through trophic relationships (Elton 1927; Strauss \& Irwin 2004). Lafferty (2008) estimates that $70 \%$ of all trophic interactions are parasitic and the antagonistic relationships between parasites and their hosts determine population and community structure in natural ecosystems (Pimm 1979; Pimm 1980a; Paine 1980; Pimm et al., 1991; Polis et al., 1997; Thompson et al., 2007; Lafferty et al., 2008; Chase 2013). Parasites require hosts for nutrition, shelter and, ultimately, survival (Tscharntke 1992; Lafferty et al., 2008; Johnson et al., 2010; Goedknegt et al., 2012; Friman \& Buckling 2013) depending on their ecological networks for development, transmission and overall fitness (Lafferty et al., 2008). 
Owing to the intimate relationship with their hosts, their complex life cycles, and the difficulties in taxonomic identification, parasites present a possibly greater challenge than free-living species in terms of estimating abundance, diversity or the implications of their associations for ecology and evolution (Dobson et al. 2008; Gómez \& Nichols 2013). As a result, parasites are often either not included in ecological research, or are portrayed solely as detrimental to free-living hosts (Nichols \& Gómez 2011). However, the intricate relationships between parasites and their hosts provide an excellent opportunity to combine and expand interdisciplinary research interests, with potentially significant advances in our understanding of complex antagonistic interactions.

\subsection{Study System}

New Zealand has a long history of isolation and a dynamic geological past (Daugherty et al. 1993). Separating from Gondwana $\sim 80$ million years ago, New Zealand's nearest continental land mass is Australia, 2000 kilometres away. Much of New Zealand's flora and fauna are remnants of their mainland ancestors, having evolved in situ, isolated from the mainland. As on many isolated islands, New Zealand harbours high levels of endemism and gigantism (Daugherty et al. 1993) with conditions promoting the evolution of many ecologically remarkable species.

Perhaps one of the most unusual is New Zealand's largest endemic moth, Aenetus virescens (Doubleday 1843; Lepidoptera: Hepialidae), a long-lived giant arboreal parasite. Male moths attain a wingspan of $150 \mathrm{~mm}$ (Grehan 1981, 1984). In flight, female moths oviposit eggs onto forest floors where first larval instars are mycophagous (Grehan 1981, 1984; Tobi et al., 1993). Subsequent larvae find, ascend and parasitise a host tree, excavating a "7"-shaped tunnel into tree heartwood. Larvae grow to $100 \mathrm{~mm}$ in length and remain enclosed in solitary tunnels for $\sim 6$ years, feeding nocturnally on host tree phloem. A feeding scar is created surrounding the tunnel entrance where phloem is extracted. Larvae construct webbing from silk and frass, which covers their tunnel entrance and feeding scar. Webbing looks much like the tree background, potentially concealing larvae from predatory parrots, the North Island kaka Nestor meridionalis septentrionalis 
(Psittaciformes: Nestoridae). Kaka consume larvae using powerful beaks to tear large chunks of wood from trees, causing extensive damage to the hosts. Larvae remain concealed throughout arboreal development, with no direct conspecific interaction until they emerge as moths to find a mate. Larvae pupate within the tunnel entrance, emerging in summer as moths with no functioning mouth parts and surviving only 1-2 days (Grehan, 1981). Despite this remarkable life history, the ecological and evolutionary relationships between the host trees, the parasitic larvae and the avian predator have not been investigated.

\subsection{Parasite aggregation}

In host individuals, parasites generally follow a common pattern of aggregation whereby many hosts have few parasites and few hosts have many (Shaw et al. 1998; Tschirren et al. 2007; Calabrese et al. 2011; Poulin \& Forbes 2011; Poulin 2013). Aggregation of parasites such as ticks, mites and nematodes is common amongst host groups of amphibians, birds, fish and mammals (Shaw et al. 1998). Host choice ultimately determines parasite fitness. Variation in parasite burden amongst conspecific hosts results from biotic factors, including host effects and infection stage of parasites, and abiotic factors, such as season (Shaw et al. 1998). Understanding what drives parasite aggregations on particular hosts is an essential foundation from which to elucidate the mechanisms driving parasite transmission and virulence (Sears et al. 2012). As such, intraspecific parasite aggregation has been a central focus in parasite ecology and evolution.

Host organisms parallel islands, comprising large complex ecological entities surrounded by uninhabitable space (Kuris et al. 1980; Southwood \& Kennedy 1983). Within an "island", available energy is a function of competitor intensity and discrete resources (Tregenza 1995; Randhawa \& Poulin 2009; Tseng \& Myers 2014) potentially leading to conspecific competition from several individuals vying for the same resources. Density-dependent regulation of species abundance is a common ecological consequence of competition for food, space or mates (Duan et al. 2013). In particular, phytophagous insects that feed on tissue inside a host tree (e.g. phloem) commonly experience density-dependent competition for food (Duan et al. 
2013). Similarly, gastrointestinal helminth populations experience density-dependent growth when sharing a host with conspecifics (Dezfuli et al. 2002). Moreover, large parasite intensities can over-burden host resources, negatively influencing parasite growth, population dynamics and genetics, whilst causing morbidity and mortality for the host (Barber 2005; Poulin 2007; Neuhäuser et al. 2010; Blasco-Costa \& Poulin 2013). Understanding intraspecific density-dependence and the mechanisms driving parasite burdens on hosts is the fundamental foundation from which to interpret all other host-parasite interactions. However, the drivers of aggregation for $A$. virescens larvae are currently unresolved.

\subsection{Host specificity}

At the community level, parasite aggregation also occurs inter-specifically. Whilst generalist parasites can exploit many host species, specialist parasites often exploit only one (Poulin \& Keeney 2008). Host specificity, the number of species a parasite can exploit relative to the larger pool available, is perhaps the most important ecological and evolutionary aspect of parasite populations (Poulin et al. 2006, 2011; Poulin \& Keeney 2008). The number of host species a parasite currently exploits provides important evolutionary insights into historical host use and associations (Poulin et al. 2006; Poulin \& Keeney 2008). The parasite's contemporary ecological niche determines the likelihood of parasite extinction if hosts become scarce, and the chance of the parasite becoming established in new areas (Poulin et al. 2006; Poulin and Keeney 2008). Interestingly, herbivorous insects are generally highly conservative in their host specificity (Funk \& Bernays 2001 ), whereas $60 \%$ of human pathogens and $80 \%$ of domestic animal pathogens, are generalist parasites capable of infecting multiple hosts (Pedersen et al. 2005). Restricted by host-parasite biogeographical and evolutionary history, and constrained by ecological and physiological boundaries (Poulin \& Keeney 2008), the degree of host specialisation is ultimately determined by a parasite's adaptations to local hosts (Gotthard et al. 2004). Determining the patterns and processes driving host specificity is therefore fundamental for understanding the role of parasites in ecosystems. 
Variation in the spatial structure of potential hosts has significant ecological and evolutionary consequences for parasite populations (Funk \& Bernays 2001). Differences in host availability and the utilisation of those hosts in separate parasite populations can lead to local adaptations and the divergence of host preferences (Gotthard et al. 2004). When faced with sporadic host availability, generalist parasites often utilise less preferred host species (Sears et al. 2012; Lootvoet et al. 2013), but are constrained by parasite dispersal and infection mode (Pedersen et al. 2005; Poulin 2013). Host species that are more abundant in the environment are likely to be encountered and parasitised most often by parasites who use hosts at random (Krasnov et al. 2004). However, parasites often locate hosts in response to stimuli (Belan \& Bull 1991; McCoy 2003) and successful infection relies on the parasites' ability to discriminate between heterogeneous hosts in fragmented populations (Théron et al. 1998).

Parasite adaptations to their local hosts is a fundamental evolutionary process that determines host-parasite specialisation (Gotthard et al. 2004). Parasites benefit directly from host nutritional quality; however, host-parasite relationships also arise from co-evolutionary arms races between host defences and a parasites' ability to circumvent these (Dawkins \& Krebs 1979; Langmore et al. 2003). Generalist parasites must invest in an array of potentially costly counter-adaptations to overcome defences presented by several hosts (Poulin 2007; Sears et al. 2012). Parasites capable of infecting multiple host species are potentially responsible for the emergence of new pests and disease both in humans and in wildlife (Pedersen et al. 2005). However, our general understanding of host specificity, beyond a few focal parasite species, is currently lacking. Identifying which hosts a parasite can use, and which traits make a host susceptible to parasites, underpins the evolution of parasite aggregation, transmission and virulence, and whether colonisation of new locations is likely (Sears et al. 2012). Host specificity is therefore particularly important for $A$. virescens, whose long arboreal life stage and restricted distribution expose them to even small perturbations in host populations. 


\subsection{Adaptive Consequences of Camouflage}

Predation is one of the most significant pressures an organism will face. With the recent inclusion of parasites as consumers in food webs, emerging evidence suggests parasites are also a specific food source for predators (Johnson et al. 2010; Goedknegt et al. 2012; Thieltges et al. 2013). The consumption of parasites is often accidental, occurring when parasites are consumed as a bi-product of their host being predated (Goedknegt et al. 2012). However, deliberate predation of parasites' free-living life stages also occurs (Goedknegt et al. 2012). Parasites that have specific predators face the same selection pressures as free-living organisms when it comes to the evolution of anti-predator adaptations.

Colour and pattern in animals play a crucial role in visual communication and are considered to have three main functions: predator avoidance, intraspecific communication and thermoregulation (Endler 1978; Mallet \& Joron 1999; Schmidt et al. 2004; Merilaita \& Lind 2005; Bond 2007). Camouflage has long been considered a seminal example of natural selection and adaptation (Darwin 1858; Wallace 1870; Poulton 1890; Bond 2007). Animals often use a combination of morphology, colour matching, disruptive patterns and items found in the environment to conceal themselves and reduce detection by predators (Cuthill et al. 2005; Stevens \& Merilaita 2009; Stevens \& Tevens 2015). The adaptive consequences of camouflage in prey organisms should result in increased survivability via reduced predation for the most cryptic individuals. However, our understanding of the adaptive consequences of camouflage has not progressed significantly since the seminal works of Cott (1940), and many of the classic taxa used as examples of adaptive camouflage remain untested (Stevens \& Merilaita 2009). Whilst numerous studies consider the degree of crypsis in avoiding initial detection by predators, few studies actively investigate whether cryptic organisms actually survive better by reducing predation (Stevens \& Merilaita 2009; Troscianko et al. 2013; Merilaita \& Dimitrova 2014).

A fundamental requirement for camouflage to function as an anti-predator adaptation is the ability to go undetected by predators, thereby increasing prey survivability. Signal detection is determined by the sensory perception and 
physiologies of the signal receivers, and is a key driver of the evolution of visual signalling (Blackledge 1998). Much work has been undertaken on avian vision and visual sensory perception. Avian colour vision allows a greater range of colour perception than human vision perceives and occurs via four visual colour cones; the perception of visual pigment via oil-droplets for spectral absorption within the eye allow colour vision across the 300-700 nanometre $(\mathrm{nm})$ wavelengths which include the ultraviolet (UV) spectrum (300-400 nm) (Church et al., 1998; Vorobyev et al., 1998; Endler \& Mielke 2005; Stoddard \& Prum 2008). However, whilst details regarding the visual perception of specific predators is lacking, few studies even consider the role of predator visual perception in assessing the use of camouflage as a defence. Sensory biases in the perception of visual signals and the sensory processing ability of the receiver indicate a discrepancy in the ability of camouflaged organisms to be hidden from all predators at one time (Blackledge 1998; Stevens \& Merilaita 2009). This indicates that camouflage as a predator avoidance adaptation is intimately linked to the signal perception of the predator(s). For $A$. virescens larvae, feeding nocturnally at their tunnel entrance may expose them to visual predators such as kaka. The webbing constructed by $A$. virescens to cover the tunnel entrance potentially conceals larvae from predating kaka; however, this has not yet been investigated.

\subsection{Male-biased Host Susceptibility}

The most pronounced differences between individuals of the same species are those occurring between sexes. However, only recently has host sex been considered an influence for susceptibility to parasites (Goble \& Konopka 1973; Alexander \& Stimson 1988; Bundy 1988). Differences between male and female hosts frequently result in differences in parasite burdens and infection status, although the mechanisms underpinning these differences remain poorly understood. In general, a precedence for male-biased susceptibility (MBS) to parasitism has been proposed by myriad studies published in the last 50 years. Among human and non-human animals, for example, the prevalence and intensity of parasitic infection is higher in males than females (Klein 2004). The primary explanation for MBS in animals is the androgen-immune system. Male organisms invest more in mating, producing 
hormones (i.e testosterone) that enhances sexual traits but simultaneously suppresses the immune system, increasing susceptibility to parasites (Zuk \& McKean 1996; Zuk \& Stoehr 2002). In the past 30 years, emerging evidence suggests males and females of dioecious plants, where male and female functions are on separate individuals, also have a similar pattern of MBS (Agren 1999). However, unlike animals, plants do not have an analogous system to the androgenimmune trade-off. Thus, if a similar pattern of MBS exists across plants and animals, then the immunosuppression caused by testosterone is not an over-arching explanation for MBS.

Female investment in offspring results from maternal adaptations to increase their own Darwinian fitness (Shaanker et al. 1988), yet offspring, by nature, take resources from females. Resources taken by one offspring cannot go to another, and offspring compete with siblings, and with their mother, for maternal resources (Shaanker et al. 1988). Seeds on plants, which are sinks for female resources, often show a negative correlation between, for example, grain number and stem mass, demonstrating genetic conflict between parents and offspring (Sadras \& Denison 2009). Similarly in animals, conflict with maternal resources is manifested via sibling rivalry. The conflict between offspring, mothers and siblings indicates an intense competition for the finite resources of females and has no parallel in males. Thus, females of animals and plants represent an already depleted pool of resources for parasites. Conversely, male hosts present an approximately full resource pool in the absence of the burden of offspring and are potentially able to support a greater number of parasites than females. The preferred host of $A$. virescens larvae is the dioecious tree Aristotelia serrata; however, whether a bias in susceptibility between host sexes exists remains unresolved. 


\subsection{Research Aims and Thesis Overview}

In this thesis, I present the first investigation of the natural history of New Zealand's largest endemic moth Aenetus virescens, the relationship with their host trees and the interactions with their avian predator. I used a system-based approach to investigate complex drivers of parasite-host interactions and the evolutionary and ecological theory therein, using $A$. virescens as a model system. Specifically, my aims were to: i) investigate the mechanisms driving parasite aggregation on hosts, ii) investigate the traits determining parasite host specificity at multiple locations, iii) test the adaptive consequences of camouflage, and iv) investigate MBS to parasites.

In Chapter 2, I investigate the mechanisms driving intraspecific parasite aggregation by first quantifying the pattern of parasite infrapopulations on individual host trees. To do this, I quantified individual host size and discuss the implications for available resources and the rate at which parasites may encounter larger hosts. I investigated long-term parasite growth to assess density-dependent competition as a possible mechanism driving parasite aggregation on individual hosts.

In Chapter 3, I investigate A. virescens larvae host specificity at multiple locations. I tested for differences in forest composition, i.e. potential hosts, quantifying tree abundance, tree size and the number of trees with parasites. Then, for the 24 most common tree species, I quantified host nutritional rewards (phloem turnover and phloem sugar content) and host defensive traits (bark thickness and wood density) as the most likely drivers of larvae host specificity. I discuss my findings in terms host traits that determine susceptibility to parasite attack and the ecological and evolutionary implications therein.

In Chapter 4, I investigate the adaptive advantages of camouflage for $A$. virescens larvae. I quantified whether larvae webbing was visually cryptic to predating kaka via spectral analysis in avian tetrahedral colour space. Next, to evaluate the adaptive advantages of crypsis, I used larvae survivability over a 26month field experiment where I manipulated the conspicuousness of webbing. I then quantified predator attack rate to assess whether cryptic webbing reduced larvae 
predation. Finally, I tested whether an alternative adaptive advantage of webbing was to aid larvae thermoregulation by assessing the temperatures inside larvae tunnels with and without webbing.

In Chapter 5, I present the first amalgamated approach testing the overarching patterns and process driving male-biased susceptibility (MBS) in both animals and plants. Specifically, I determined the magnitude of MBS by compiling the largest database of host-parasite pairings to date $(n=461)$ from 188 studies. I used a meta-analysis to evaluate the degree of MBS amongst individual host species, and at the higher taxonomic level of family. I then developed a novel theoretical model to explain MBS in animals and plants, hypothesising that parasites compete with offspring for female resources. I tested the model predictions using independent empirical data from the dioecious host tree- $A$. virescens larvae system. In this chapter, I illustrate that reduced defences via immunosuppression is not a universal cause of MBS by comparing defences between male and female hosts. Finally, I discuss the implications this novel unified model has for current and future research.

In Chapter 6, I synthesise the results of the previous chapters. Primarily, the results of this thesis present the first investigation of the ecological and evolutionary relationships between host trees, parasitic $A$. virescens larvae and their avian predators. The results herein support existing theories of parasite aggregation and host specificity from a novel perspective. Furthermore, my results support a newly emerging paradigm shift in the theory of animal camouflage evolution and present the first unified explanation for male-biased susceptibility to parasites in animals and plants. This thesis presents an extensive foundation for future research to further explore this remarkable study system and to use the theories herein to guide multidisciplinary parasite research. 


\section{Chapter 2. Drivers of aggregation in a novel arboreal parasite: the influence of host size and infra- populations}

Adapted from:

Yule \& Burns (2014) Drivers of aggregation in a novel arboreal parasite: the influence of host size and infrapopulations. International Journal for Parasitology, 45(2-3), $197-202$ 


\subsection{Abstract}

As a novel arboreal parasite, New Zealand's largest endemic moth, Aenetus virescens, is a biological oddity. With arguably the most unusual lepidopteran life history on Earth, larvae grow to $100 \mathrm{~mm}$, spending $~ 6$ years as wood-boring parasites feeding on host tree phloem. Parasite fitness is a product of host suitability. Parasite discrimination between heterogeneous hosts in fragmented populations shapes parasite aggregation. I investigated whether $A$. virescens aggregation among hosts occurs randomly (target area effect), or if larvae use hosts based on host quality (ideal free distribution). Using long-term larval growth as an indicator of energy intake, I examined $A$. virescens aggregation in relation to host size and infrapopulation. Using a generalised linear model, the relationship between parasite intensity and host tree size was analysed. Reduced major axis regression was used to evaluate $A$. virescens growth after 1 year. Linear mixed-effects models inferred the influence of parasite infra-population on parasite growth, with host tree as a random factor. Results indicate parasite intensity scaled positively with host size. Furthermore, parasite growth remained consistent throughout ontogeny regardless of host size or parasite infra-population. Aenetus virescens aggregation among hosts violates the ideal free distribution hypothesis, occurring instead as a result of host size, supporting the target area effect. 


\subsection{Introduction}

Host quality determines parasite growth, reproductive success and survival (Théron et al. 1998; Poulin et al. 2003; Barber 2005; Tschirren et al. 2007; Poulin \& Forbes 2011). Parasites aggregate, whereby few parasites infect many hosts and many parasites infect few hosts (Shaw et al. 1998; Tschirren et al. 2007; Calabrese et al. 2011; Poulin \& Forbes 2011; Daniels et al. 2013). Understanding parasite distribution patterns among hosts is crucial for accurate modelling of population dynamics (Poulin 2000). Among hosts, exposure and susceptibility to parasites is heterogeneous, correlating with host size and age, driving parasite aggregation (Grutter and Poulin 1998; Shaw et al., 1998; Poulin 2013).

Heterogeneity in host nutritional quality also influences parasite aggregation (Poulin et al., 2003; Tschirren et al., 2007). Endoparasites encounter finite resources (Poulin, 2007, 2013; Randhawa and Poulin 2009; Daniels et al., 2013) and intensitydependent resource competition reduces host quality, regulating parasite body size (Poulin 1999; Barber 2005). Furthermore, parasite dissemination and infection mode influence host selection (Lester 2012; Poulin 2013). Overall parasite success relies on discriminating between heterogeneous hosts in fragmented populations (Théron et al., 1998). Host selection is particularly significant for New Zealand's largest endemic moth, Aenetus virescens (Doubleday 1843; Lepidoptera: Hepialidae), whose larvae are long-term parasites of host trees Aristotelia serrata.

Aenetus virescens has a remarkable lepidopteran life history. Male moths attain a wingspan of $150 \mathrm{~mm}$ (Fig. 2.2.1. A) (Grehan 1981, 1984). For most insects, adult females select hosts for larvae by choosing plants on which to oviposit eggs. Adult females of $A$. virescens, however, oviposit eggs onto forest floors where first larval instars are mycophagous (Grehan 1981, 1984; Tobi et al., 1993). Subsequent larvae select, ascend and parasitise a host tree, excavating a "7"-shaped tunnel into tree heartwood. Larvae remain enclosed for $\sim 6$ years, growing to $100 \mathrm{~mm}$ (Fig. 2.2.1. B) and feeding on phloem tissue at the tunnel entrance (Grehan 1981, 1983, 1984; Tobi et al., 1993). A feeding scar is created around the tunnel opening (Fig. 2.2.1. C). Constructions of silk and frass webbing cover these feeding scars, behind which larvae feed nocturnally (Grehan 1984; Tobi et al., 1993). Webbing potentially 
conceals larvae from their visual predators, North Island kaka Nestor meridionalis septentrionalis, the only extant avian predators capable of excavating larvae from inside their host trees. Larvae remain solitary and concealed throughout arboreal development, with no direct conspecific interaction until mating after emergence. Larvae pupate within the tunnel entrance, emerging as moths in summer with no functioning mouth parts and survive only 1-2 days (Grehan 1981). Despite this remarkable parasitic life history, the ecological role of $A$. virescens, in particular the relationship with host trees, is poorly understood.

(a)

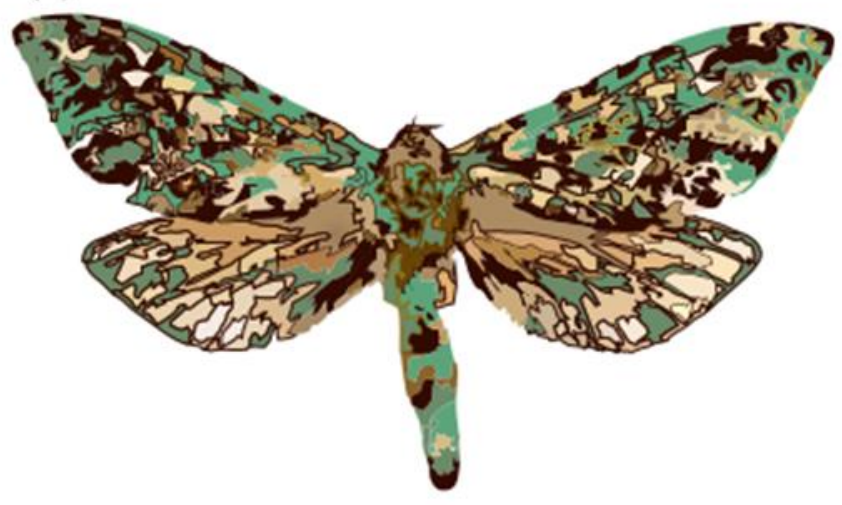

$1 \mathrm{~cm}$ (b)

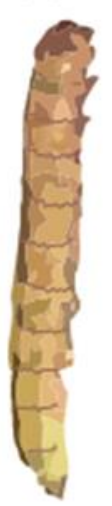

$1 \mathrm{~cm}$ (c)

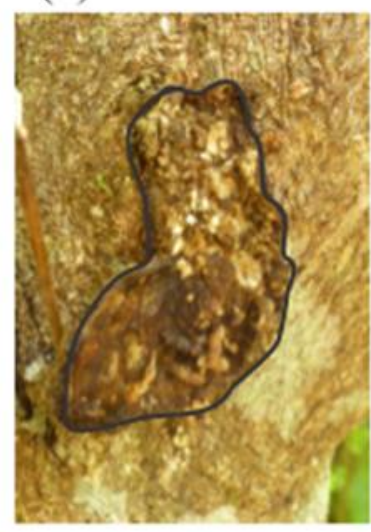

$1 \mathrm{~cm}$

Figure 2.2.1. New Zealand's largest endemic moth, Aenetus virescens. (a) Adult moth (female), (b) parasitic arboreal-phase larva, and (c) parasite feeding scar on Aristotelia serrata host tree, covered with silk and frass webbing (webbing is outlined).

Host trees parallel islands, comprising large, complex, ecological entities surrounded by uninhabitable space (Kuris et al., 1980; Southwood and Kennedy 1983). The Ideal Free Distribution theory (IFD) predicts free and mobile organisms use habitat "islands" providing the greatest reward (Tregenza 1995; Tyler and Gilliam 
1995; Stewart and Komers 2012; Williams et al., 2013). Beneath tree outer bark, phloem carries photosynthates from the canopy to the roots (Högberg et al., 2001; Zwieniecki et al., 2004; Pompon et al., 2011). Phloem thickness scales strongly with tree diameter (Amman 1969; Speights and Conway 2010; Hölttä et al., 2013; Davis and Hofstetter 2014), is a nutrient-rich resource (Pompon et al., 2011), and the sole foodstuff of $A$. virescens. Conversely, the hydrostatic conductance of phloem is reduced in older, larger trees, indicating a decreased flow of photosynthates (Yoder et al., 1994; Bond 2000; Hölttä et al., 2013). Whilst larger trees present a greater quantity of food, nutritional quality is likely reduced. If $A$. virescens discriminate between hosts with varying nutritional benefits, smaller trees will have a greater parasite intensity. However, the Target Area Effect (TAE) states larger habitable "islands" merely represent larger targets more easily intercepted by randomly dispersing individuals (MacArthur and Wilson 1967; Lomolino, 1990; Matter and Roland 2003). For example, larger hosts present larger surface areas, and thus an increased chance of discovery by parasites (Poulin 2013). Moreover, larger hosts tend to be older and have had longer to accrue parasites (Poulin 2013). If $A$. virescens disperse randomly, larger trees will have greater parasite intensity than smaller trees.

Energy intake scales with body size and growth (see Lindstedt et al., 1986; Keeley and Grant 1995; Greenleaf et al., 2007). When A. virescens feed, phloem tissue is masticated and the contents ingested. The resulting feeding scar is indicative of the phloem quantity consumed by an individual larva. If larvae can discern host quality, trees with greater available energy per unit of phloem will have greater parasite intensity. Moreover, parasites consuming equivalent phloem quantities should have increased growth rates in higher quality hosts (Barber 2005; Tseng and Myers 2014). Within an "island", however, available energy is a function of competitor intensity and discrete resources (Tregenza 1995; Randhawa and Poulin 2009; Tseng and Myers 2014) with host saturation reducing host quality (Kaplan and Denno, 2007). Parasite intensity scales negatively with parasite growth when parasite-to-host body size ratio is high (Poulin 1999; Barber 2005; Lagrue and Poulin 2008). Moreover, large parasite intensities over-burden host resources, 
negatively influencing parasite growth, population dynamics and genetics (Barber 2005; Poulin 2007; Neuhäuser et al., 2010; Blasco-Costa and Poulin 2013).

Aenetus virescens body size is relatively small compared with host trees; however, feeding scars extensively wound phloem, remaining after parasites vacate the host. Cumulative wounding from multiple feeding scars per host produces largescale phloem disruption. Phloem girdling often results from $A$. virescens parasitism, whereby feeding scars cover the full circumference of the tree, cutting off any downwards flow of photosynthates. Intensive phloem wounding, particularly phloem girdling, reduces xylem water transportation altering water-use efficiency and flow of photosynthates, decreasing tree fitness (Zwieniecki et al., 2004; Zvereva et al., 2010). Multiple feeding scars may therefore dramatically reduce host quality in terms of nutrient availability to individual larva. Aenetus virescens growth could thus experience intensity-dependent regulation.

Here, I investigate whether $A$. virescens aggregation among hosts occurs randomly (TAE) or if larvae use hosts based on reward (IFD). Using long-term larval growth as a measure of host quality, I examine how host size and parasite intensity drive $A$. virescens aggregation. I answer the following three questions: i) Does parasite intensity scale positively with host size? ii) Does parasite body size scale positively with feeding scar size? and iii) Is parasite growth intensity-dependent?

\subsection{Materials and Methods}

\subsubsection{Study Sites}

Data were collected in Zealandia, a mainland island reserve at the southern tip of the North Island, New Zealand $\left(41^{\circ} 28^{\prime} \mathrm{S}, 174^{\circ} 74 \mathrm{~W}\right)$. The climate is mild and temperate, with elevations ranging from 160 to 380 metres above sea level (Blick et al., 2008). Primary forest was cleared for agriculture in the late 1800s (Blick et al., 2008; Burns et al., 2012; Burns, 2012) and Zealandia now comprises 225 ha of successional broadleaf/conifer forest enclosed by a mammal-resistant fence. Introduced mammalian predators were eradicated in 2000, aiding the re-introduction of native fauna and flora, and in particular native birds (Burns 2012). Dominant broadleaf evergreen trees such as Coprosma spp. (Rubiaceae), Melicytus ramiflorus 
(Violaceae), Pseudopanax arboreus (Araliaceae), Dysoxylum spectabile (Meliaceae) and Schefflera digitata (Araliaceae) are common. The dense understorey comprises tree ferns, Cyathea spp. (Cyatheaceae), shrubs including Brachyglottis repanda (Asteraceae), Geniostoma rupestre var. Ianguifolium (Loganiaceae) and Piper excelsum (Piperaceae), and vines such as Rhipogonum scandens (Ripogonaceae).

Zealandia has an established population of Aristotelia serrata (Elaeocarpaceae), which, as shown in chapter 3 , are the preferred host species of $A$. virescens larvae. Whilst other tree species are also used as hosts by larvae in Zealandia, $A$. serrata had significantly more larvae than any other host species (Chapter 3).

\subsubsection{Data collection and analysis}

\subsubsection{Parasite intensity and host size}

Parasite intensity, the number of parasites per host tree including old, disused tunnels and live larvae, plus tree height and tree diameter at breast height (DBH = $1.25 \mathrm{~m}$ ) were recorded for $A$. serrata host trees $(n=63)$. Host size was calculated as tree bole surface area. Tree boles were considered conical in shape, starting wide at the base and tapering towards the crown. The surface area of the cone-shaped tree bole was calculated as

$$
H S=(\pi r s)+\left(\pi r^{2}\right)
$$

where $H S$ is host size, $r$ is radius of tree bole, and $s$ is slant of tree bole. Slant was calculated as

$$
s=\sqrt{ }\left(r^{2}+h^{2}\right)
$$

where $h$ is height. Tree canopies were discounted from host size calculations as parasites were observed in tree boles only. A generalised linear model (GLM, family = poisson) was used to analyse the influence of host size on parasite intensity. 


\subsubsection{Parasite size and feeding scar}

Long-term growth of parasites was established via feeding scars. I measured the size $\left(\mathrm{cm}^{2}\right)$ of all feeding scars with larvae present in February 2013 (feeding scar, FA2013; $n=116$ ) using image analysis software, ImageJ (Rasband 2014). A random subset of parasites $(n=18)$ was selected to analyse the relationship between feeding scar and larval body size. Larvae were weighed in the field. Head width, tail width, body length and volume were measured in the laboratory using digital callipers. A pairwise correlation matrix deduced the allometric relationships between larval body parts. Principal component analysis (PCA) provided a representative parasite body size (PCA1). I used reduced major axis regression (RMA) to analyse the relationship between feeding scar size and PCA1, providing slope and intercept parameters ( $\pm 95 \%$ confidence limits $[C I])$. The slope and intercept parameters estimated parasite body size for all feeding scars where larvae were not collected ( $n$ =98). One year later (FA2014), 57 feeding scars were randomly selected and their size $\left(\mathrm{cm}^{2}\right)$ measured. The RMA slope and intercept parameters were used to provide an estimated size for FA2014 based on calculated parasite sizes. A paired t-test compared estimated FA2014 with actual FA2014. A non-significant difference allowed the feeding scar to become a proxy for parasite body size. Larval growth was calculated across ontogeny as the difference between FA2013 and FA2014 ( $\mathrm{n}=$ 57). Using RMA slope and intercept parameters ( $\pm 95 \% \mathrm{Cl})$, I established whether the relationship between FA2013 and FA2014 differed from isometry (1:1). A simple linear regression analysed the influence of host size on parasite growth.

\subsubsection{Parasite growth and intensity}

Within an "island", available energy is a function of competitor intensity and discrete resources (Tregenza 1995; Randhawa and Poulin 2009; Tseng and Myers 2014), with host saturation reducing host quality (Kaplan and Denno 2007). Accounting for non-independence of parasites sharing a host, the influence of "tree" was included as a random factor in linear mixed-effects models. Models analysed whether larval growth was influenced by i) the sum of conspecific larvae in a host tree, ii) the sum of conspecific larvae occurring above a focal individual in a host tree 
(upstream competitors), iii) the summed size of feeding scars with larvae present in a host tree, and iv) the summed size of feeding scars with larvae present, occurring above the focal individual in a host tree. For each model a Likelihood ratio test was executed to elucidate the degree of influence each independent variable had on larval growth. All variables, excluding "tree", were square root transformed to meet normality assumptions.

Data analysis was performed in $\mathrm{R}$ version 3.1.0. (R Core Team 2014). The package "smatr" was used to conduct RMA analyses (Warton et al., 2012). Linear mixed-effects models, including the likelihood ratio tests, were executed using the package "Ime4" (Bates et al., 2014). Statistical significance was determined by $P$ values less than 0.05 .

\subsection{Results}

2.4.1. Parasite intensity and host size

Data were obtained from $63 A$. serrata host trees. Host size varied from 25.53 $\mathrm{m}^{2}$ to $247.20 \mathrm{~m}^{2}$ (median $\left.=107.11 \mathrm{~m}^{2}\right)$. Parasite prevalence was high with $73 \%$ of hosts harbouring at least one parasite. The number of feeding scars per infected host varied from 1-64 (median =4), with 306 feeding scars in total. Live parasites accounted for 116 feeding scars, varying from 1-19 (median =2) per infected host. Furthermore, 190 feeding scars were post-parasite (the parasite had emerged), varying from $1-45$ (median $=2.5)$ per infected host. Parasite intensity, i.e. parasite infra-population, scaled positively with host size (GLM: $F=31.49$, df $=55, P<0.001$, Fig. 2.4.1). 


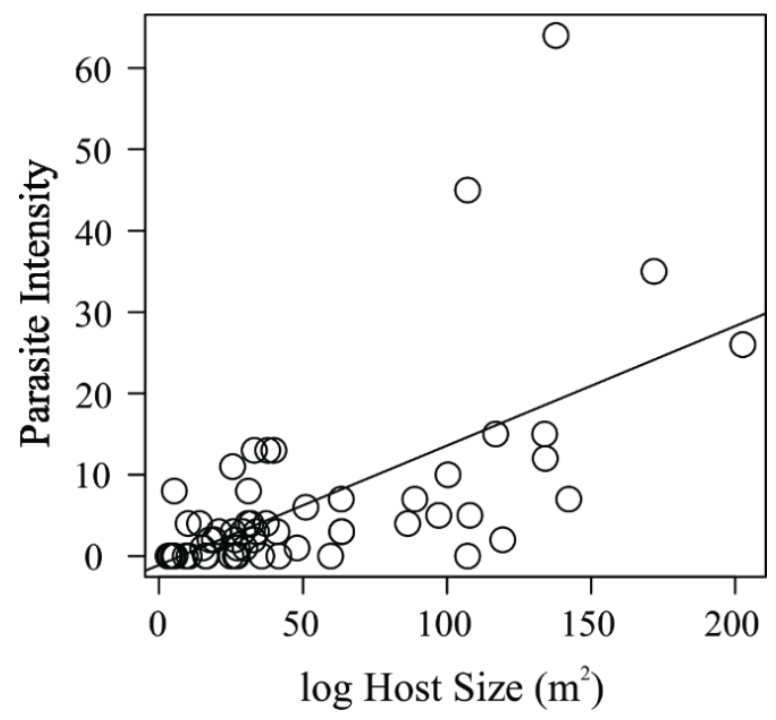

Figure 2.4.1. Influence of Aristotelia serrata host tree size on arboreal parasite Aenetus virescens infra-population.

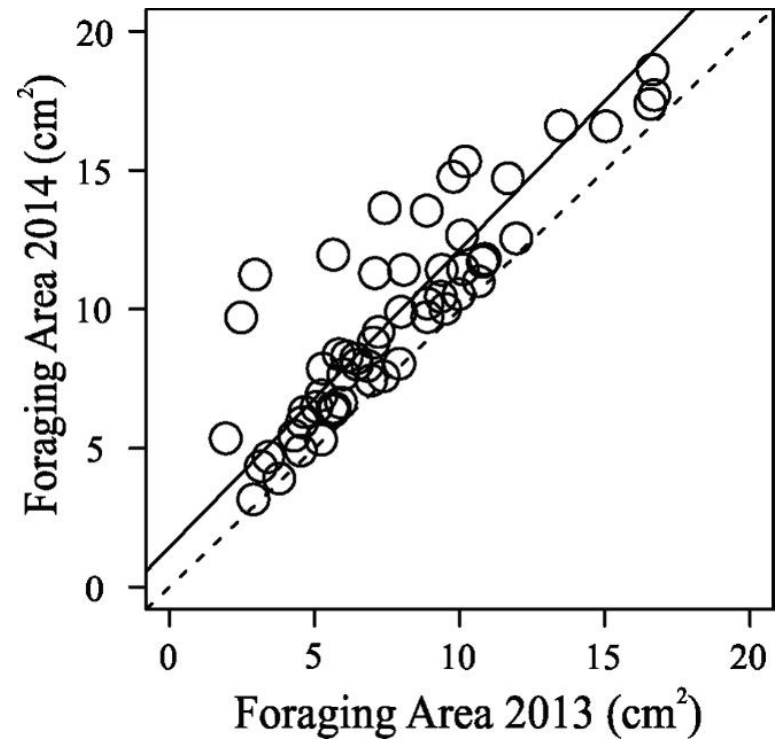

Figure 2.4.2. Growth of arboreal parasite Aenetus virescens feeding scar (proxy for parasite body size) between year 1, February 2013 (feeding scar, FA2013) and year 2, February 2014 (FA2014). Dashed line shows isometry (1:1). Solid line shows reduced major axis (RMA). 


\subsubsection{Parasite size and feeding scar}

Parasite weight, head width, tail width, length and volume scaled positively with each other, with parasite length being the principal component (PCA1) (Supplementary Fig. A1). Parasite body size scaled positively with FA2013 $\left(r^{2}=\right.$ $0.75 ; \mathrm{F}=50.63, \mathrm{df}=16, \mathrm{P}<0.001)$. Based on calculated parasite body size from FA2013, I found no significant difference between estimated and actual FA2014 ( $\mathrm{t}=$ $-0.0001, \mathrm{df}=17, \mathrm{P}=0.99$ ). Henceforth, feeding scar was a proxy for parasite body size. The size of FA2013 scaled positively with the size of FA2014 (RMA regression: $r^{2}=0.76 ; P<0.001$, Fig. 2.4.2.). All feeding scars increased in size between year 1 and year 2, differing from isometry. However, growth did not vary significantly with parasite size, remaining consistent throughout ontogeny (slope: $1.07,95 \% \mathrm{Cl}=$ 0.94-1.22; intercept: $1.44,95 \% \mathrm{Cl}=0.24-2.63$, Fig. 2.4.2.).

\subsubsection{Parasite growth and intensity}

Host size did not significantly influence larval growth $\left(r^{2}=0.02 ; F=2.034\right.$, df $=$ $55, P=0.16)$. Furthermore, when host tree was included as a random factor, parasite intensity did not influence parasite growth. Growth was not significantly influenced by the sum of conspecific larvae in a host tree $(X 2=0.0536, d f=4, P=$ 0.81, Fig. 2.4.3.a), the sum of conspecific larvae occurring above a focal individual in a host tree (upstream competitors) $(\mathrm{X} 2=2.614$, $\mathrm{df}=4, \mathrm{P}=0.10$, Fig. 2.4.3.b), the summed size of all feeding scars with larvae present per host tree $(X 2=0.2615$, $d f=$ $4, P=0.60$, Fig. 2.4.3.c), nor the summed size of all feeding scars with larvae present occurring above a focal individual per host tree $(X 2=2.3312$, df $=4, P=$ 0.12, Fig. 2.4.3.d). 

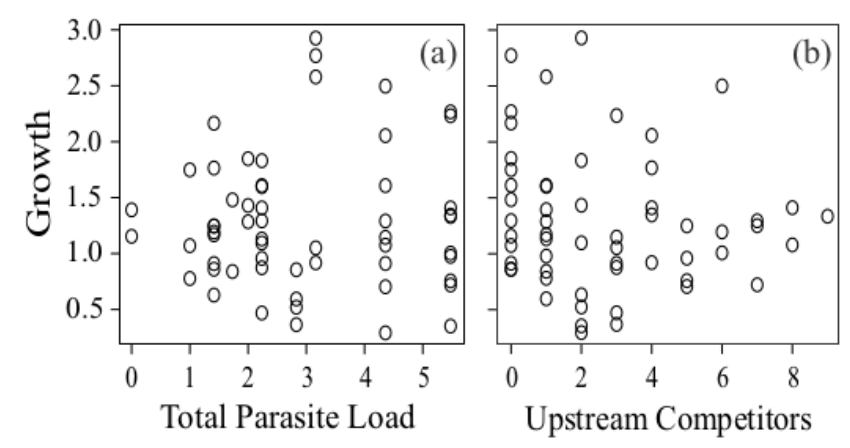

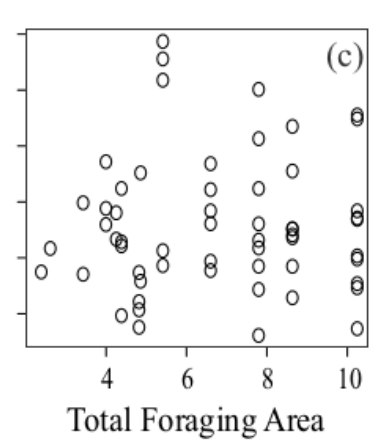

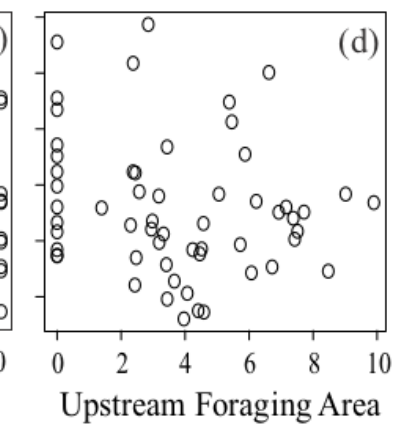

Figure 2.4.3. Influence of infra-population on Aenetus virescens arboreal parasite growth on (a) the sum of conspecific larvae in an Aristotelia serrata host tree, (b) the sum of conspecific larvae occurring above a focal individual in a host tree (upstream competitors), (c) the summed size of all feeding scars with larvae present in a host tree, and (d) the summed size of feeding scars with larvae present, occurring above the focal individual in a host tree.

\subsection{Discussion}

To my knowledge, drivers of aggregation in the novel arboreal parasite, Aenetus virescens have not been empirically tested. In particular, I investigated whether parasites discriminate between heterogeneous host trees in a fragmented population (IFD), or if parasites randomly discover hosts (TAE). Furthermore, I assessed the influence of host size and parasite intensity on parasite aggregation. Despite their unique life history, $A$. virescens followed the common parasite distribution pattern of few hosts harbouring many parasites, and many hosts harbouring few parasites (Shaw et al., 1998; Tschirren et al., 2007; Calabrese et al., 2011; Poulin and Forbes 2011; Poulin, 2013). I found that parasite intensity scaled positively with host size. A prominent pattern in parasite ecology, parasite intensity commonly scales with host size (see Poulin 2000, 2005; Poulin and Morand 2000). In particular, a meta-analysis of 76 different fish host-parasite relationships found positive correlations between host size and parasite intensity, although relationship significance varied (Poulin 2000). Larger hosts are predominantly older individuals with larger external surface areas and accrue greater parasite intensities through 
time and space (see Poulin and Morand 2000; Poulin 2013). Tree size is strongly correlated with tree age (Bond, 2000), whilst phloem thickness strongly scales with tree diameter (Amman 1969; Speights and Conway 2010; Hölttä et al., 2013; Davis and Hofstetter 2014). Phloem translocates nutritionally rich photosynthates from the canopy to the roots (Högberg et al., 2001; Zwieniecki et al., 2004; Pompon et al., 2011) and is the sole foodstuff for $A$. virescens. However, decreased phloem hydraulic conductance in older, larger trees denotes a reduced flow of photosynthates (Yoder et al., 1994; Bond 2000; Hölttä et al., 2013). From the perspective of a parasite, larger trees offer a greater quantity, but a reduced quality of food, while smaller trees offer greater available energy per unit of phloem. Aenetus virescens are 10-35 mm in length when commencing the parasitic arboreal stage (Grehan 1983), and parasites were recorded in host trees as small as $10 \mathrm{~mm}$ $\mathrm{DBH}$. Parasites in smaller host trees negate the energetic cost required to ascend and parasitise larger hosts, as often in larger trees, larvae must climb above already existing tunnels to find space to excavate their own tunnel. If $A$. virescens discriminate between heterogeneous hosts, smaller trees should host greater parasite intensities than larger trees owing to the increased nutritional quality and lower energetic costs. With $A$. virescens intensity being greater on larger hosts, I conclude that host choice is not based on host quality, but on random discovery. This supports the TAE assumptions that larger "islands" support a greater number of individuals when dispersal is random (MacArthur and Wilson 1967; Lomolino 1990; Matter and Roland 2003).

Significant scaling relationships were identified between $A$. virescens weight, head width, tail width, length and volume. Concurrently, $A$. virescens body size scaled positively with feeding scar size, allowing feeding scars to become proxies for parasite body sizes. Feeding scar size is a direct representation of the quantity of phloem a larvae consumes. Host tree size did not significantly influence feeding scar size, indicating that larvae consumed equivalent phloem quantities regardless of the host tree size. In all organisms, growth is a result of energy intake (see Lindstedt et al., 1986; Keeley and Grant 1995; Greenleaf et al., 2007). The parasite growth rate should increase in nutritionally advantageous hosts (Barber 2005). Conversely, I found that $A$. virescens growth rate remained consistent throughout ontogeny 
regardless of parasite size or host tree size. However, phloem nutritional quality may not solely influence parasite aggregation and growth. Competitive hosts generally have improved immune responses and may minimise nutritional availability to parasites (Barber 2005). Aristotelia serrata are considered competitive owing to a fast-growing life strategy, particularly in early ontogeny (Dawson and Lucas 2011). Moreover, factors such as temperature likely play a significant role in $A$. virescens development. Temperature is vital to ectotherm growth, influencing physiological and morphological characteristics (Poulin and Latham 2003; Kingsolver et al., 2006). Smaller trees may provide less insulation from temperature extremes, potentially fostering disadvantageous microclimates in parasite tunnels. Nevertheless my results suggest that larger $A$. serrata size does not equate to increased host quality for parasites. The parasites are therefore not aggregating as a response to increased host quality, further supporting random dispersal as suggested by the TAE.

The IFD suggests that infra-population size is a function of the available energy of an "island" (Tregenza 1995; Stewart and Komers 2012; Williams et al., 2013). "Islands", in particular, have finite resources and conspecific interactions are exacerbated by more individuals vying for the same resources (Tregenza 1995; Randhawa and Poulin 2009; Tseng and Myers 2014). In turn, host saturation reduces host quality (Kaplan and Denno 2007). Infra-population size scales negatively with parasite body size in most host-parasite interactions (see Ikeda 1979; Rankin and Borden 1991; Poulin 1999, 2007; Barber 2005; Lagrue and Poulin 2008; Neuhäuser et al., 2010; Duan et al., 2013; Blasco-Costa and Poulin 2013). I found $A$. virescens intensity varied remarkably between hosts, ranging from 1-19 (median $=2$ ) feeding scars with parasites present. Additionally, post-parasite feeding scars remained as wounds in phloem and ranged from 1-45 (median $=2.5$ ) per host. Feeding scars are substantial wounds in trees. Cumulative wounding from multiple feeding scars per host produces large-scale phloem disruption. Phloem girdling often results from $A$. virescens parasitism, whereby feeding scars cover the full circumference of the tree, cutting off any downwards flow of photosynthates. Intensive phloem wounding, particularly phloem girdling, reduces xylem water 
transportation, altering water-use efficiency and decreasing tree fitness (Zwieniecki et al., 2004). Multiple feeding scars may therefore dramatically reduce host quality.

Any parasite above another conspecific in a tree bole interrupts the flow of energy travelling downwards. Parasite nutrient supply is potentially limited by competitors, thus growth is expected to decrease with an increasing infra-population. Contrastingly, my results indicate that $A$. virescens growth did not significantly correlate with parasite intensity. Furthermore, growth of an individual parasite did not significantly correlate with the number of parasites directly above that individual. Additionally, the summation of all feeding scars per host did not significantly correlate with parasite growth. Moreover, feeding scars directly above a focal individual did not significantly influence its growth. Consequently, $A$. virescens growth, representative of energy intake, is not intensity-dependent. In fish hostparasite relationships, low parasite intensities do not constrain parasite growth, whereas large parasite intensities produce resource competition and intensitydependent growth (Poulin and Morand 2000; Poulin 2005; Saldanha et al., 2009). Although $A$. virescens are small bodied compared with host trees and occur in relatively low numbers (post- and present feeding scars, median $=4$ ), the sizeable feeding scars were expected to decrease host quality. Interestingly, some tree species are tolerant of consumer attacks, inducing adaptive responses regulating nutrient availability and internal resource allocation (Haukioja and Koricheva 2000; Stowe et al., 2000). These adaptations potentially mitigate fitness reductions to host trees from increased feeding scars; therefore, trees with greater parasite intensities may be no less advantageous to parasites if trees are tolerant of such damage.

I conclude that the $A$. virescens relationship with their host trees contradicts the IFD, which predicts "island" resources are a function of conspecific competition. The IFD proposes that individuals actively select "islands" providing greater rewards. However, my results indicate that larger hosts provide greater available space but no increase in obtainable energy for parasites, as shown by parasite growth. Therefore, it is unlikely that $A$. virescens are aware of available resources when disseminating to a host tree. More likely, as stated by the TAE, larger hosts provide a larger target more easily intercepted by larvae, leading to greater infra-populations. Furthermore, 
parasite growth was not influenced by any level of parasite intensity. Thus, increasing infra-population does not reduce host quality, further violating assumptions of the IFD. In conclusion, $A$. virescens aggregation among hosts supports the TAE. Larger trees randomly accumulate greater parasite intensities because they are larger, older presences in the landscape. 


\section{Chapter 3. Host defensive traits explain parasite host specificity in multiple populations}

Adapted from:

Yule, KJ and Burns, KC (In review) Host defensive traits explain parasite host specificity in multiple populations. Evolutionary Ecology 


\subsection{Abstract}

Host specificity, the number of host species a parasite can exploit, varies dramatically between species and populations. Whilst generalist parasites exploit many host species, specialist parasites often exploit only one. However, the patterns and processes determining host specificity remain unresolved. Here, I investigate host specificity of New Zealand's largest endemic moth, Aenetus virescens (Lepidoptera: Hepialidae), a long-lived arboreal parasite. Unusually, larvae determine individual fitness by using host trees. Larvae excavate solitary tunnels into tree heartwood, living $\sim 6$ years feeding on host tree phloem. I investigated whether: i) forest composition differed between sites, giving larvae a different pool of species from which to choose hosts, ii) tree abundance predicted the number of trees parasitised such that larvae were using the most common species as hosts, and iii) tree traits such as rewards (phloem turnover and phloem sugar content) or defences (bark thickness and wood density) determined the number of trees parasitised. Results indicate forest composition differed significantly between sites. Larvae were generalist parasites and used specific tree species as preferred hosts regardless of tree abundance. The number of trees parasitised significantly increased as bark thickness decreased, indicating that trees with thinner bark were more susceptible to parasite attack. Conversely, no significant relationship was found between the number of parasitised trees and any other tree trait. Overall, $A$. virescens preferentially attack host species with reduced defensive traits. This study suggests host external defences are the primary mechanism driving host specificity across sites with differing host pools. 


\subsection{Introduction}

Antagonistic relationships between parasites and their hosts shape populations and ecosystems (Gómez \& Nichols 2013; Olsson-Pons et al. 2015). Parasites rely solely on their hosts for nutrition, protection and overall fitness (Barber 2005; Poulin 2007; Tschirren et al. 2007; Poulin \& Forbes 2011). Host specificity, the number of species a parasite can exploit relative to the larger pool available, is perhaps the most important ecological and evolutionary aspect of parasite species and populations (Poulin et al. 2006; Poulin \& Keeney 2008). The number of host species a parasite currently exploits provides important evolutionary insights into historical host use and associations, whilst also defining the contemporary ecological niche of the parasite, its likelihood of extinction and the risk of the parasite becoming problematic in new areas (Poulin et al. 2006; Poulin \& Keeney 2008). Whilst generalist parasites have evolved to exploit multiple host species, specialist parasites are often confined to only one (Pedersen et al. 2005; Poulin et al. 2006; Poulin \& Forbes 2011). Restricted by host-parasite biogeographical and evolutionary history and constrained by ecological and physiological boundaries (Poulin \& Keeney 2008), the degree of host specialisation is ultimately determined by a parasite's adaptations to local hosts (Gotthard et al. 2004). Determining the patterns and processes driving host specificity is therefore fundamental for understanding the role of parasites in ecosystems.

The evolution of host specialisation in insects predominantly results from adult females' choosing oviposition sites (Thompson \& Pellmyr 1991; Gotthard et al. 2004). For New Zealand's largest endemic moth, Aenetus virescens (Doubleday 1843) (Lepidoptera: Hepialidae), female moths scatter eggs across the forest floor, where first larval instars spend several months as mycophages (Grehan 1981, 1984; Tobi et al. 1993). Subsequent larvae locate host trees, ascend the tree bole and excavate a "7"-shaped tunnel into tree heartwood. Larvae grow to $100 \mathrm{~mm}$ and remain solitary inside their tunnels for up to 6 years (Fig. 3.2.1a). Webbing made from silk and frass is constructed over the tunnel entrance (Fig. 3.2.1b) behind which larvae feed nocturnally on host tree phloem (Fig. 3.2.1c) (Grehan, 1981, 1983, 1984; Tobi et al., 1993). Large parrots, Nestor meridionalis (Gmelin 1788) (Psittaciformes: 
Nestoridae) (Fig. 3.2.1d), consume these larvae by using powerful beaks to tear large chunks of wood from trees (Fig. 3.2.1e). Larvae pupate within their tunnel entrance, emerging as moths in summer with no functioning mouth parts, surviving only 1-2 days (Fig. 3.2.1f) (Grehan, 1981). Aenetus virescens (hereafter "larvae") are the ideal parasite to investigate the patterns and processes of host specificity owing to their long history of isolation with potential hosts. When New Zealand split from Gondwana $~ 80$ million years ago, larvae were separated from their mainland ancestors, facilitating the tandem evolution of larvae and hosts. Despite this remarkable lifestyle and evolutionary history, the mechanisms driving larvae host specificity are unknown.
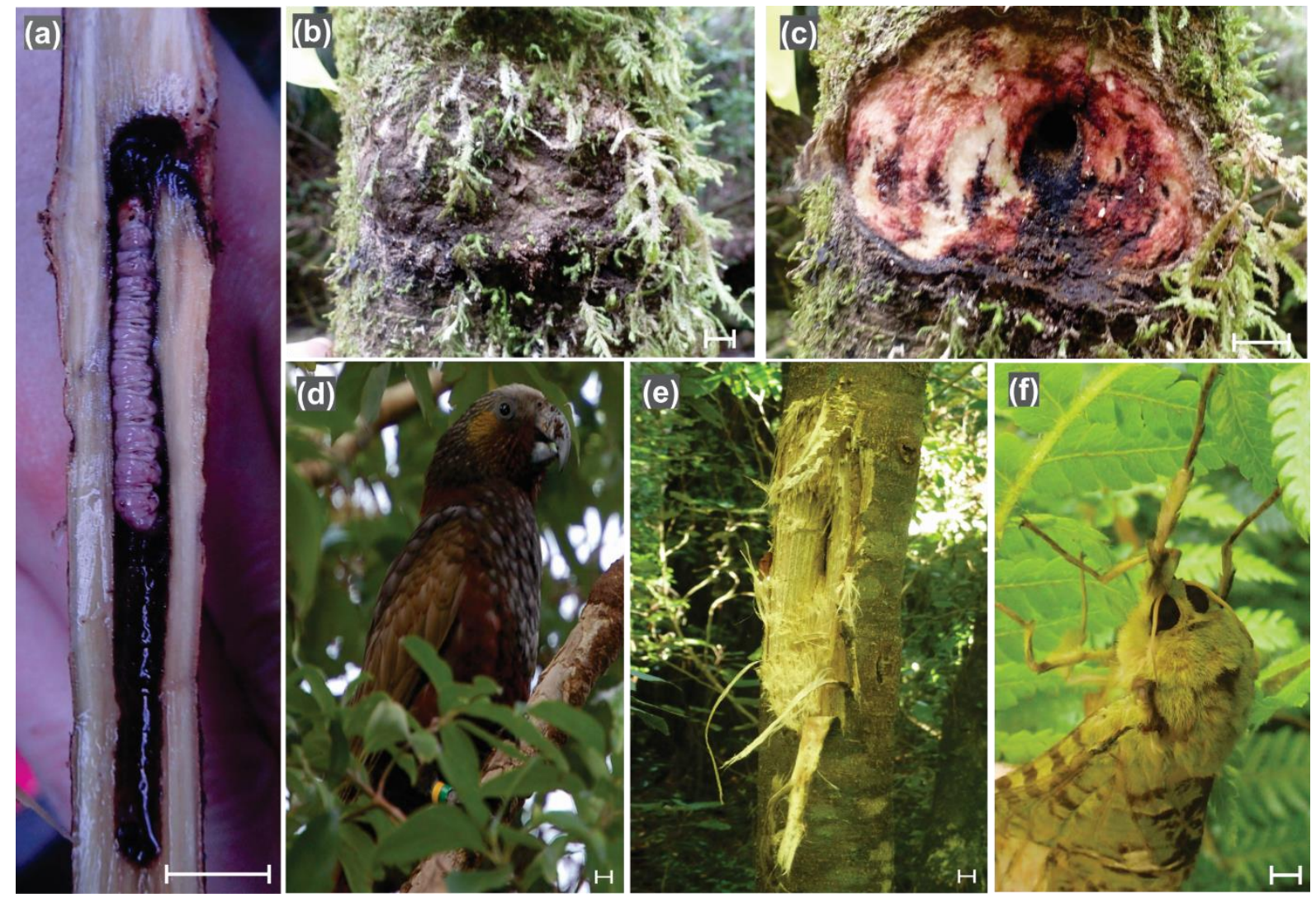

Figure 3.2.1. (a) Aenetus virescens larvae inside a host sapling, (b) Webbing covering the tunnel entrance of $A$. virescens, (c) Feeding scar surrounding the tunnel entrance after webbing was removed, (d) the North Island Kaka, Nestor meridionalis septentrionalis, (e) Damage to host trees after kaka excavated an $A$. virescens larvae, (f) Adult female A. virescens. All scale bars represent $1 \mathrm{~cm}$. 
Variation in the spatial structure of potential hosts has significant ecological and evolutionary consequences for parasite populations (Funk \& Bernays 2001). Differences in host availability and the utilisation of those hosts in separate parasite populations can lead to local adaptations and the divergence of host preferences in each population (Gotthard et al. 2004). When faced with sporadic host availability, generalist parasites often utilise less preferred host species, constrained only by parasite dispersal and infection mode (Pedersen et al. 2005; Poulin 2013). Host species that are more abundant in the environment are likely to be encountered and parasitised most often by parasites who use hosts at random (Krasnov et al. 2004). However, parasites often locate hosts in response to stimuli (Belan \& Bull 1991; McCoy 2003) and success relies on the parasites' ability to discriminate between heterogeneous hosts in fragmented populations (Théron et al. 1998).

Often, when multiple suitable hosts are available, parasites preferentially attack particular host species that maximise parasite fitness (Sears et al. 2012; Lootvoet et al. 2013). Parasite adaptations to their local hosts is a fundamental evolutionary process determining host-parasite specialisation (Gotthard et al. 2004). For example, parasites benefit directly from host nutritional quality. Optimal foraging theory predicts that, given an equal handling time, food items with the greatest energy rewards will be chosen more often than low energy food items (Lozano 1991). Phloem sap is composed mainly of sugars, and represents relative carbohydrate concentration for sap-feeders (Martinez-Trinidad et al. 2010). The flow of phloem sap through the vascular cambium and phloem sugar content represents the potential nutritional benefit to sap feeders and varies between tree species. However, host-parasite relationships also arise from co-evolutionary arms-races between host defences and a parasites' ability to circumvent these (Dawkins \& Krebs 1979; Langmore et al. 2003). Generalist parasites must invest in an array of potentially costly counter-adaptations to overcome defences presented by several hosts (Poulin 2007; Sears et al. 2012). Tree traits such as wood density and bark thickness can provide trees with a physical defence against attack (Abell et al. 2012; Santini et al. 2012). However, insect wood-borers commonly circumvent host tree defences, interrupt the flow of water and nutrients and syphon valuable photosynthates that deprive the tree of food (Hanks et al. 1999). Identifying which 
traits make a host susceptible to parasites underpins the evolution of a parasite's aggregation, transmission and virulence at existing, and new, locations.

Here, I present the first investigation of larvae host specificity across three sites in the Wellington region of New Zealand's North Island. Specifically, I ask whether: i) forest composition differed between sites such that parasites had a different pool of species from which to choose hosts, ii) tree abundance predicted the number of trees parasitised such that parasites were using the most common species as hosts, and iii) tree traits such as rewards (phloem turnover and phloem sugar content) or defences (bark thickness and wood density) determined the number of trees parasitised.

\subsection{Materials and Methods}

\subsubsection{Study Sites}

Data were collected between November 2013 and January 2015 from three sites in the Wellington region of New Zealand's North Island that had established populations of $A$. virescens larvae (Fig. 3.3.1). Each site consisted of mixed broadleaf-podocarp forest (Table 31 ). Dominant broadleaf evergreen trees such as Coprosma spp. (Rubiaceae), Melicytus ramiflorus (Violaceae), Pseudopanax arboreus (Araliaceae) and Schefflera digitata (Araliaceae) were common between sites (Table 3 1). The dense understorey at each site included tree ferns Cyathea spp. (Cyatheaceae), woody shrubs including Brachyglottis repanda (Asteraceae), Geniostoma rupestre var. languifolium (Loganiaceae) and Piper excelsum (Piperaceae), and vines such as Rhipogonum scandens (Ripogonaceae) (Table 31 ). Larvae are not found at higher altitudes, so all data were collected from within valleys and surrounding hillsides ranging from 160 to $800 \mathrm{~m}$ above sea level at each site. All sites were similar in topography and hydrology, with annual rainfall for the region averaging $1200 \mathrm{~mm}$ and annual temperatures averaging $13.2{ }^{\circ} \mathrm{C}$ (Macara 2014). 


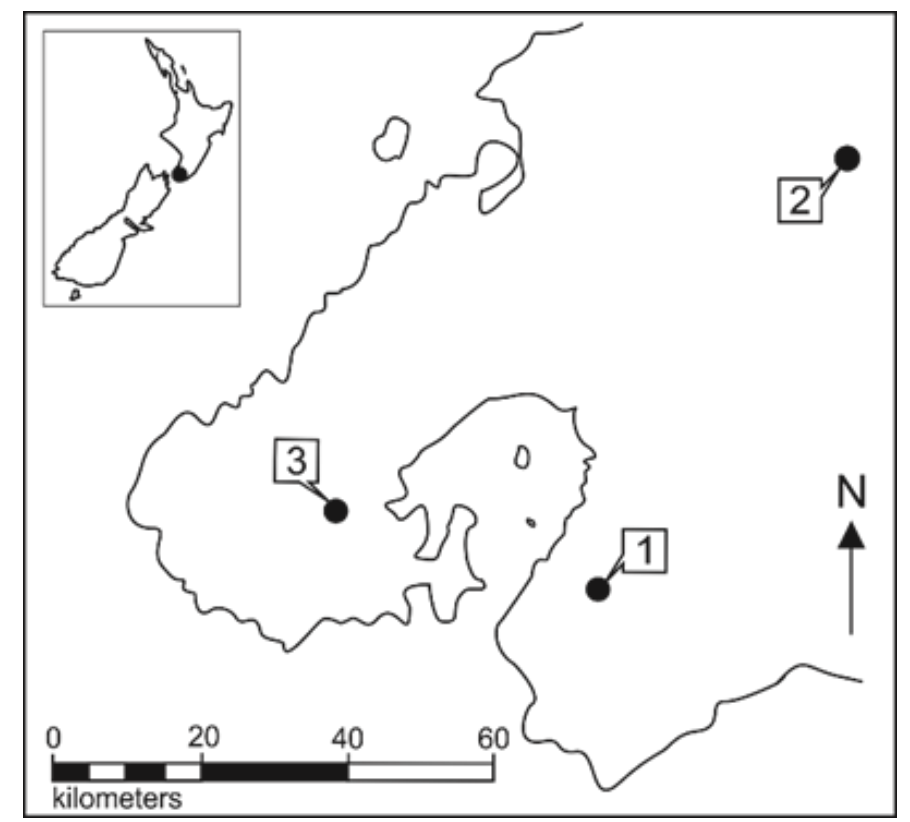

Figure 3.3.1. Wellington Region of New Zealand's North Island. Site 1: Butterfly Creek, East Harbour Regional Park; Site 2: Kaitoke Regional Park, Pakuratahi; and Site 3: Zealandia, Karori.

\subsubsection{Does forest composition differ between sites?}

To compare the similarity of forest composition between sites, I recorded the presence and abundance of tree species using $10 \mathrm{~m} \times 10 \mathrm{~m}$ forest plots at random locations within each site (Site 1: $n=25$, Site 2: $n=31$, Site 3: $n=35$ ). The abundance of tree species from each forest plot was used to calculate the BrayCurtis similarity index between site pairings. The similarity index is a continuum between 0 and 1 , whereby 0 indicates sites were completely different in composition and 1 indicates sites had the same composition. Tree presence and abundance was pooled from all forest plots for each site for analysis 


\subsubsection{Does tree abundance predict number of trees parasitised?}

From all forest plots, I identified 2318 individual trees that could be visually assessed for the presence or absence of larvae. Larvae were identified via the presence of a feeding scar covered by intact webbing. Larvae reconstruct damaged webbing over the course of one night, and therefore intact webbing represents live larvae (Yule \& Burns, Unpubl.). To account for the available habitat each tree presents to larvae, I quantified the surface area of the cone-shaped tree bole for each individual tree as follows

$$
H S=(\pi r s)+\left(\pi r^{2}\right)
$$

where $H S$ is host size, $r$ is radius of tree bole and $s$ is slant of tree bole. Slant was calculated as

$$
s=\sqrt{ }\left(r^{2}+h^{2}\right)
$$

where $h$ is height (Yule \& Burns, 2015). Tree canopies were discounted in host surface-area calculations because parasites were observed in tree boles only.

To assess whether the number of trees with parasites increased with tree abundance, I ran a generalised linear mixed effects model (GLMM) with binomial (logit) link function for each of the three sites. For the binomial response variable, I used number of trees with parasites and number of trees without parasites, with tree abundance as the fixed factor. I accounted for the habitat available to larvae by including the sum of tree surface area for each species at each site as a random factor in each of the models. 


\subsubsection{Do tree traits determine number of trees parasitised?}

Whether tree traits determined host specificity of larvae was investigated across all three sites. To ensure sufficient sample sizes, I eliminated tree species with $<20$ individuals from further analysis. This left the 24 most abundant tree species, representing $95.5 \%$ of all trees across all sites for inclusion in analysis (Table 31 ). For these 24 species, I assessed the tree traits that I considered most likely to influence larvae host choice: tree rewards that might encourage parasite attacks via nutritional benefit to parasites, such as phloem turnover and phloem sugar content; and tree defences that might prevent parasite attacks, such as bark thickness and wood density. Twenty individual trees per species $>6 \mathrm{~cm}$ diameter at breast height $(\mathrm{DBH}=$ $1.25 \mathrm{~m}$ ) were randomly selected and phloem sugar content, phloem turnover, wood density and bark thickness were assessed for each tree $(n=480)$.

To assess phloem turnover for each tree species, I used cambial electrical resistance (CER) to measure the movement of electrical currents through tree vascular tissue. CER is indicative of phloem sugar turnover, i.e how quickly phloem sugars move through the cambium (Plamping et al. 2009; Gričar 2012), replenishing food supply for phloem feeders. Following Martinez-Trinidad et al. (2010) CER was assayed using a stainless steel electrode digital multimetre (Digitech QM1323). Electrodes were inserted approximately $5 \mathrm{~mm}$ into the phloem layer, spaced $20-\mathrm{mm}$ apart vertically. The minimal electrical resistance (k-ohms) from 5 minutes of pulsed electrical currents was recorded from DBH on the north- and south-facing aspects of each tree $(n=480)$. The mean \pm standard error $(\mathrm{SE})$ was calculated for each tree species. CER was sampled on dry, sunny days during summer (November-January) with ambient air temperature between $18^{\circ} \mathrm{C}$ and $21^{\circ} \mathrm{C}$. Only single-trunk trees with no visible damage were assessed.

To assess phloem sugar content for each tree species, the outer bark layers were removed at DBH from North and South aspects of each tree $(n=480)$ and a 10 $\mathrm{mm} \times 10 \mathrm{~mm} \times 2 \mathrm{~mm}$ piece of vascular cambium was excised. Samples were placed in vials with $1 \mathrm{ml}$ of distilled water and stored at room temperature for 48 hours. I then took $0.3 \mathrm{ml}$ of solution from each vial and calculated solute concentration using an Atago Pocket (PAL-O6S) hand-held refractometer. Salinity values were converted to 
Degrees Brix ( $1^{\circ} \mathrm{Bx}=1$ gram dissolved solid in $100 \mathrm{~g}$ of solution) and the means $\pm \mathrm{SE}$ calculated for each tree species.

To assess bark thickness, a $20 \mathrm{~mm} \times 10 \mathrm{~mm}$ rectangle of bark was excised from north- and south-facing aspects of each tree at $\mathrm{DBH}(n=480)$ before collecting tissue for phloem sugar concentration (see above). To ensure I considered only the defensive traits of bark, I assessed the thickness of the outer cork layers only. Whilst measurements of bark thickness usually incorporate the vascular cambium (Cornelissen et al. 2003), I excluded vascular cambium from bark thickness analysis because I considered this a reward to larvae and not part of the defensive traits of bark. Digital callipers were used to measure bark thickness $(\mathrm{mm})$ at opposite ends of each bark piece and mean \pm SE was calculated for each tree species.

To assess wood density, I excised a $20 \mathrm{~mm} \times 10 \mathrm{~mm} \times 10 \mathrm{~mm}$ piece of wood from the north- and south-facing aspects of each tree $(n=480)$ at DBH. To minimise damage to trees, pieces of wood were collected from where bark and phloem tissue had been previously removed. The fresh mass of each wood sample was determined and wood volume was measured using the water displacement method, after which the sample was oven-dried for at least 48 hours at $70^{\circ} \mathrm{C}$ and weighed. Wood density (in $\mathrm{g} \mathrm{cm}^{-3}$ ) was determined as wood dry mass over fresh wood volume (Poorter et al. 2010). The mean \pm SE wood density was calculated for all 24 species.

To assess whether any of the four tree traits explained the number of trees that were parasitised by larvae, I ran generalized linear mixed effects model (GLMM) with binomial (logit) link function. I included number of trees with parasites and number of trees without parasites as the binomial response variable and phloem turnover, phloem sugar content, bark thickness and wood density as fixed factors. I accounted for habitat available to larvae by including the sum of tree surface area for each species at each site as the random variable. 
All statistical analyses were performed in $\mathrm{R}$ version 3.2.3 ( $\mathrm{R}$ Core Team 2015), with package "Ime4" version 1.1-7 (Bates et al. 2014) for the mixed effects models.

\subsection{Results}

\subsubsection{Does forest composition differ between sites?}

Across all three sites, I identified 2318 individual trees from 48 endemic and one non-endemic species, spanning 32 woody plant families (Table 31 ). Overall, 12 tree species were present at all three sites and 16 tree species were present in at least two sites (Table 31 ). To assess whether forest composition differed between sites, I used the Bray-Curtis similarity index for each of the site pairings. All three sites were considerably different in forest species composition. These results indicate that Sites 2 and 3 were the least similar, with a $70 \%$ difference in tree composition between sites (Fig. 3.4.1). The contrast between Sites 1 and 3, meanwhile, indicated a 68\% difference in forest composition (Fig. 3.4.1). The sites that were most similar to each other in forest composition were Sites 1 and 2; however, there was still a 59\% difference in site forest composition (Fig. 3.4.1).

\subsubsection{Does tree abundance predict number of trees parasitised?}

Overall, 196 individual trees from 20 tree species had larvae present. At each of the three sites, I assessed whether the number of trees parasitised was explained by tree abundance per species. These results indicate larvae are not attacking tree species based on abundance. I found no significant relationship between tree abundance and the number of trees that were attacked at Site 1 (GLMM: $z=1.413$, $P>0.10$, Fig. 3.4.1.), Site 2 (GLMM: $z=-0.899, P>0.10$, Fig. 3.4.1.), or Site 3 (GLMM: $z=-0.393, P>0.10$, Fig. 3.4.1.). 
Table 3.1. All woody species abundances and number of individuals attacked by Aenetus virescens in the Wellington region of New Zealand's North Island. Species listed in order of total abundance. Site 1: Butterfly Creek; Site 2: Kaitoke Regional Park; and Site 3: Zealandia. (g) in "Endemic" column indicates endemic genus. Numbers (\#) identify species in Figures 3.4.1 and 3.5.1.

\begin{tabular}{|c|c|c|c|c|c|c|c|c|c|c|c|c|c|}
\hline \multirow[t]{3}{*}{ \# } & \multirow[t]{3}{*}{ Species } & \multirow[t]{3}{*}{ Family } & \multirow{3}{*}{$\begin{array}{l}\text { Endemic } \\
\text { species? }\end{array}$} & \multicolumn{4}{|c|}{ Tree Abundance } & \multicolumn{4}{|c|}{ \# Trees Attacked } & \multirow[t]{3}{*}{ Included? } & \multirow[t]{3}{*}{ Reason } \\
\hline & & & & Site & & & & Site & & & & & \\
\hline & & & & 1 & 2 & 3 & Total & 1 & 2 & 3 & Total & & \\
\hline 1 & Coprosma grandifolia & Rubiaceae & yes & 84 & 31 & 186 & 301 & - & - & 4 & 4 & Yes & $>20$ single stemmed adults \\
\hline 2 & Coprosma robusta & Rubiaceae & yes & 2 & 5 & 176 & 183 & - & - & 4 & 4 & Yes & $>20$ single stemmed adults \\
\hline 3 & Melycitus ramiflorus & Violaceae & yes & 51 & 83 & 47 & 181 & 1 & - & 2 & 3 & Yes & $>20$ single stemmed adults \\
\hline 4 & Hedycarya arborea & Monimiaceae & yes & 74 & 52 & 30 & 156 & 3 & - & - & 3 & Yes & $>20$ single stemmed adults \\
\hline 5 & Piper excelsum & Piperaceae & yes & - & 4 & 122 & 126 & - & - & - & - & Yes & $>20$ single stemmed adults \\
\hline 6 & Schefflera digitata & Araliaceae & yes & - & 66 & 50 & 116 & - & 1 & 3 & 4 & Yes & $>20$ single stemmed adults \\
\hline 7 & $\begin{array}{l}\text { Geniostoma rupestre var. } \\
\text { Ligustrifolium }\end{array}$ & Loganiaceae & yes & 31 & 13 & 67 & 111 & - & 1 & - & 1 & Yes & $>20$ single stemmed adults \\
\hline 8 & Beilschmiedia tawa & Lauraceae & yes & 5 & 105 & - & 110 & - & - & - & - & Yes & $>20$ single stemmed adults \\
\hline 9 & Myrsine australis & Primulaceae & yes & 66 & - & 35 & 101 & 5 & - & - & 5 & Yes & $>20$ single stemmed adults \\
\hline 10 & Brachyglottis repanda & Asteraceae & yes & 13 & 2 & 73 & 88 & - & - & 2 & 2 & Yes & $>20$ single stemmed adults \\
\hline 11 & Dacrycarpus dacrydioides & Podocarpaceae & yes & 71 & 9 & 5 & 85 & - & - & - & - & Yes & $>20$ single stemmed adults \\
\hline 12 & Carpodetus serratus & Rousseaceae & yes & 27 & 30 & 12 & 69 & 23 & 27 & 7 & 57 & Yes & $>20$ single stemmed adults \\
\hline
\end{tabular}




\begin{tabular}{|c|c|c|c|c|c|c|c|c|c|c|c|c|c|}
\hline 13 & Pseudopanax arboeus & Araliaceae & yes $(g)$ & 4 & 29 & 36 & 69 & - & - & 1 & 1 & Yes & $>20$ single stemmed adults \\
\hline 14 & Fuscospora truncata & Nothofagaceae & yes & 38 & 22 & - & 60 & 14 & 8 & - & 22 & Yes & $>20$ single stemmed adults \\
\hline 15 & Fuscospora solandri & Nothofagaceae & yes & 55 & 1 & - & 56 & 30 & 1 & - & 31 & Yes & $>20$ single stemmed adults \\
\hline 16 & Nestigis cunninghamii & Oleaceae & yes & 55 & - & - & 55 & 2 & - & - & 2 & Yes & $>20$ single stemmed adults \\
\hline 17 & Dysoxylum spectabile & Meliaceae & yes & - & 1 & 53 & 54 & - & - & - & - & Yes & $>20$ single stemmed adults \\
\hline 18 & Weinmannia racemosa & Cunoniaceae & yes & 21 & 31 & - & 52 & - & - & - & - & Yes & $>20$ single stemmed adults \\
\hline 19 & Elaeocarpus dentatus & Elaeocarpaceae & yes & 28 & 7 & 15 & 50 & - & - & - & - & Yes & $>20$ single stemmed adults \\
\hline 20 & Aristotelia Serrata & Elaeocarpaceae & yes & - & - & 46 & 46 & - & - & 40 & 40 & Yes & >20 single stemmed adults \\
\hline 21 & Fuchsia excorticata & Onagraceae & yes & 1 & - & 38 & 39 & - & - & - & - & Yes & $>20$ single stemmed adults \\
\hline 22 & Pittosporum eugeniodes & Pittosporaceae & yes & 3 & 13 & 20 & 36 & - & - & 1 & 1 & Yes & $>20$ single stemmed adults \\
\hline 23 & Pseudowintera axillaris & Winteraceae & yes $(g)$ & 2 & 34 & - & 36 & - & - & - & - & Yes & $>20$ single stemmed adults \\
\hline 24 & Knightia excelsa & Proteaceae & yes & 10 & 23 & - & 33 & - & - & - & - & Yes & $>20$ single stemmed adults \\
\hline 25 & Pittosporum tenuifolium & Pittosporaceae & yes & 6 & - & 13 & 19 & - & - & - & - & No & no single stems/not enough adults \\
\hline 26 & Lophomyrtus Bullata & Myrtaceae & yes $(g)$ & 15 & - & 3 & 18 & - & - & - & - & No & small shrub \\
\hline 27 & Olearia rani var. colorata & Asteraceae & yes & 17 & - & - & 17 & - & - & - & - & No & no single stems \\
\hline 28 & Coprosma rotundifolia & Rubiaceae & yes & 15 & - & - & 15 & - & - & - & - & No & small shrub \\
\hline 29 & Myoporum laetum & Scrophulariaceae & yes & - & - & 15 & 15 & - & - & 5 & 5 & No & not enough adults \\
\hline 30 & Prumnopitys ferruginea & Podocarpaceae & yes & 11 & 1 & - & 12 & - & - & - & - & No & small shrub \\
\hline 31 & Pseudopanax crassifolius & Araliaceae & yes & 9 & - & 2 & 11 & - & - & - & - & No & not enough adults \\
\hline 32 & Coprosma lucida & Rubiaceae & yes & - & - & 10 & 10 & - & - & - & - & No & not enough adults \\
\hline 33 & Sophora microphylla & Fabaceae & yes & - & - & 8 & 8 & - & - & - & - & No & no single stems \\
\hline
\end{tabular}




\begin{tabular}{|c|c|c|c|c|c|c|c|c|c|c|c|c|c|}
\hline 34 & Corynocarpus laevigatus & Corynocarpaceae & yes & - & - & 7 & 7 & - & - & - & - & No & small shrub \\
\hline 35 & Entelea arborescens & Malvaceae & yes & - & - & 7 & 7 & - & - & - & - & No & small shrub \\
\hline 36 & Pennantia corymbosa & Pennantiaceae & yes & - & 5 & 1 & 6 & - & - & - & - & No & small shrub \\
\hline 37 & Coprosma areolate & Rubiaceae & yes & 5 & - & - & 5 & - & - & - & - & No & small shrub \\
\hline 38 & Dacrydium cupressinum & Podocarpaceae & yes & 2 & 2 & 1 & 5 & - & - & - & - & No & not enough adults \\
\hline 39 & Fuscospora fusca & Nothofagaceae & yes & - & 2 & 3 & 5 & - & 1 & 3 & 4 & No & no single stems/not enough adults \\
\hline 40 & $\begin{array}{l}\text { Plagianthus regius subsp. } \\
\text { Regius }\end{array}$ & Malvaceae & yes $(\mathrm{g})$ & - & - & 5 & 5 & - & - & 2 & 2 & No & no single stems/not enough adults \\
\hline 41 & $\begin{array}{l}\text { Alectryon excelsus subsp. } \\
\text { Excelsus }\end{array}$ & Sapindaceae & yes & - & - & 3 & 3 & - & - & 2 & 2 & No & no single stems/not enough adults \\
\hline 42 & Leucopogon fasciculatus & Ericaceae & yes & 3 & - & - & 3 & - & - & - & - & No & small shrub \\
\hline 43 & $\begin{array}{l}\text { Coprosma macrocarpa } \\
\text { subsp. minor }\end{array}$ & Rubiaceae & yes & - & - & 2 & 2 & - & - & - & - & No & small shrub \\
\hline 44 & Griselinia lucida & Griseliniaceae & yes & - & - & 2 & 2 & - & - & - & - & No & not enough adults \\
\hline 45 & Halocarpus kirkii & Podocarpaceae & yes $(g)$ & - & - & 2 & 2 & - & - & - & - & No & small shrub \\
\hline 46 & Hoheria angustifolia & Malvaceae & yes $(g)$ & 2 & - & - & 2 & - & - & - & - & No & no single stems/not enough adults \\
\hline 47 & Solanum laciniatum & Solanaceae & no & - & - & 2 & 2 & - & - & - & - & No & small shrub \\
\hline 48 & Cordyline australis & Asparagaceae & yes & - & - & 1 & 1 & - & - & - & - & No & not enough adults \\
\hline 49 & Hebe stricta var. stricta & Plantaginaceae & yes & - & - & 1 & 1 & - & - & 1 & 1 & No & no single stems/not enough adults \\
\hline
\end{tabular}




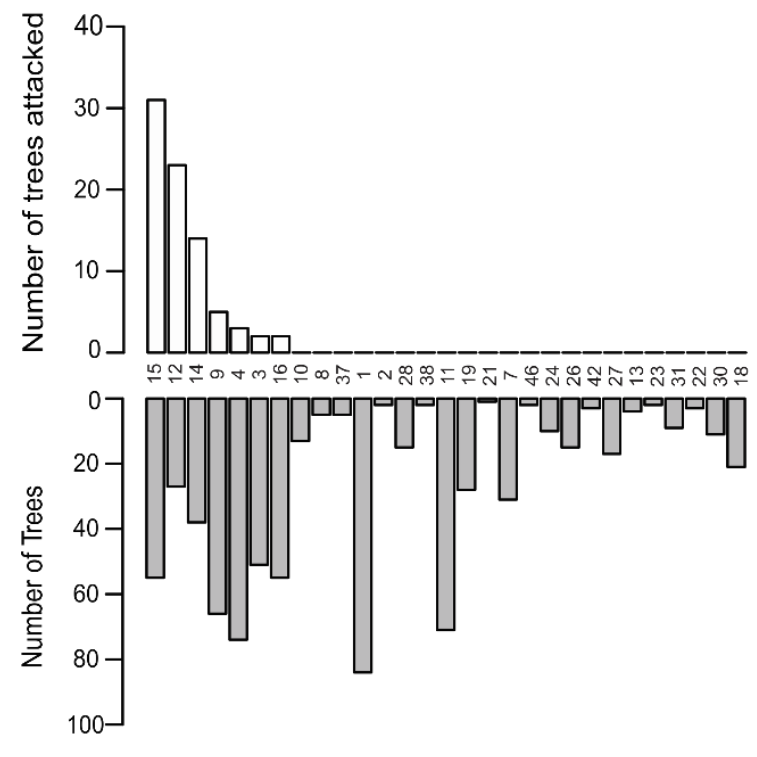

(b)

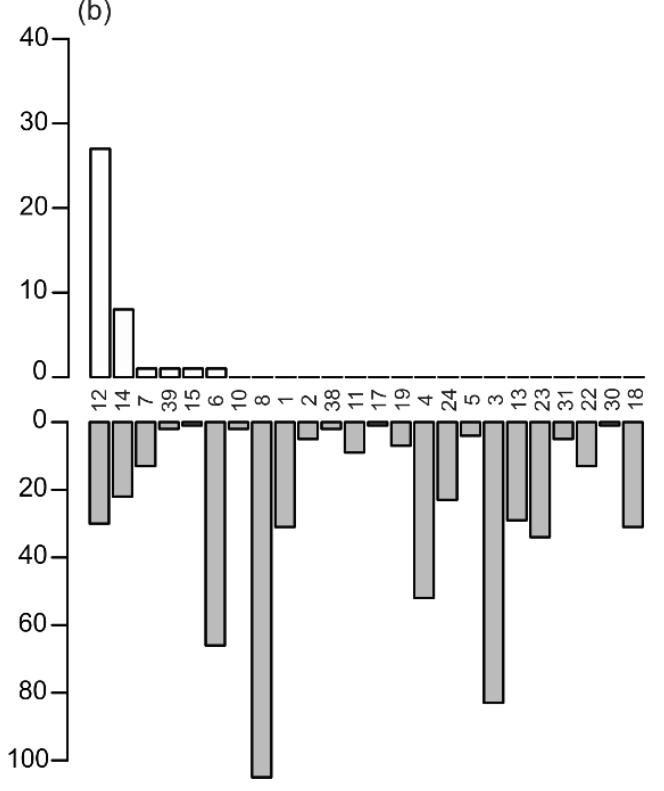

(c)

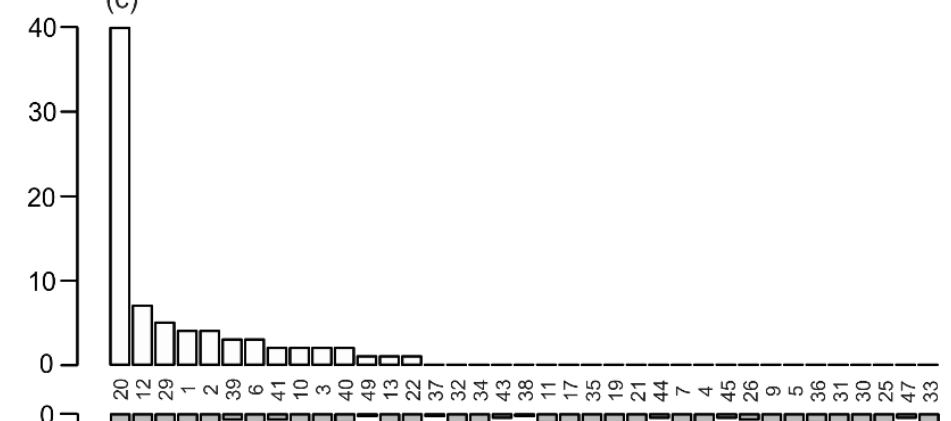

Figure 3.4.1. Y1: Total number of trees with parasites in ranked order of decreasing abundance, and Y2: Total number of trees. (a) Site 1: Butterfly Creek ( $n=30$ tree species). (b) Site 2: Kaitoke Regional Park $(n=24$ tree species). (c) Site 3: Zealandia $(n=36$ tree species). X-axis provides the number assigned to each tree species as listed in Table 3.1. 


\subsubsection{Do tree traits determine the number of trees parasitised?}

To test if the rewards parasites received from host trees determined the number of trees parasitised, I assessed phloem turnover and phloem sugar content of 24 potential host species. However, neither phloem turnover nor phloem sugar content explained the number of trees per species that were parasitised. No significant relationship was found between the number parasitised trees per species and phloem turnover $(z=1.019, P>0.10$, Fig. 3.5.1.) and no significant relationship between the number trees per species that were parasitised and phloem sugar content $(z=1.671, P>0.05$, Fig. 3.5.1.).

To test whether the number of parasitised trees per species was determined by tree defences, I assessed bark thickness and wood density of 24 potential host species. Wood density did not explain the number of trees per species that were parasitised. No significant relationship was observed between the number of trees per species that were parasitised and wood density $(z=-0.379, P>0.10$, Fig 3.5.1.). However, bark thickness did explain the number of trees per species that were parasitised. I found a significant negative relationship between the number of parasitised trees per species and bark thickness $(z=-2.112, P<0.05$, Fig. 3.5.1.). As bark thickness increased, the number of trees that were parasitised decreased.

\subsection{Discussion}

The patterns and processes determining larvae host specificity were assessed across three different sites. Although 28 out of 49 tree species were present at more than one site, I found forest composition differed substantially between sites. The pool of potential tree species from which larvae use a host were therefore different between sites. From these pools of potential hosts, the most abundant tree species were not parasitised most often, indicating that parasites are not using hosts that are more likely to be encountered. One explanation for this is that larvae may attack hosts at random, with only the successful attacks on host 

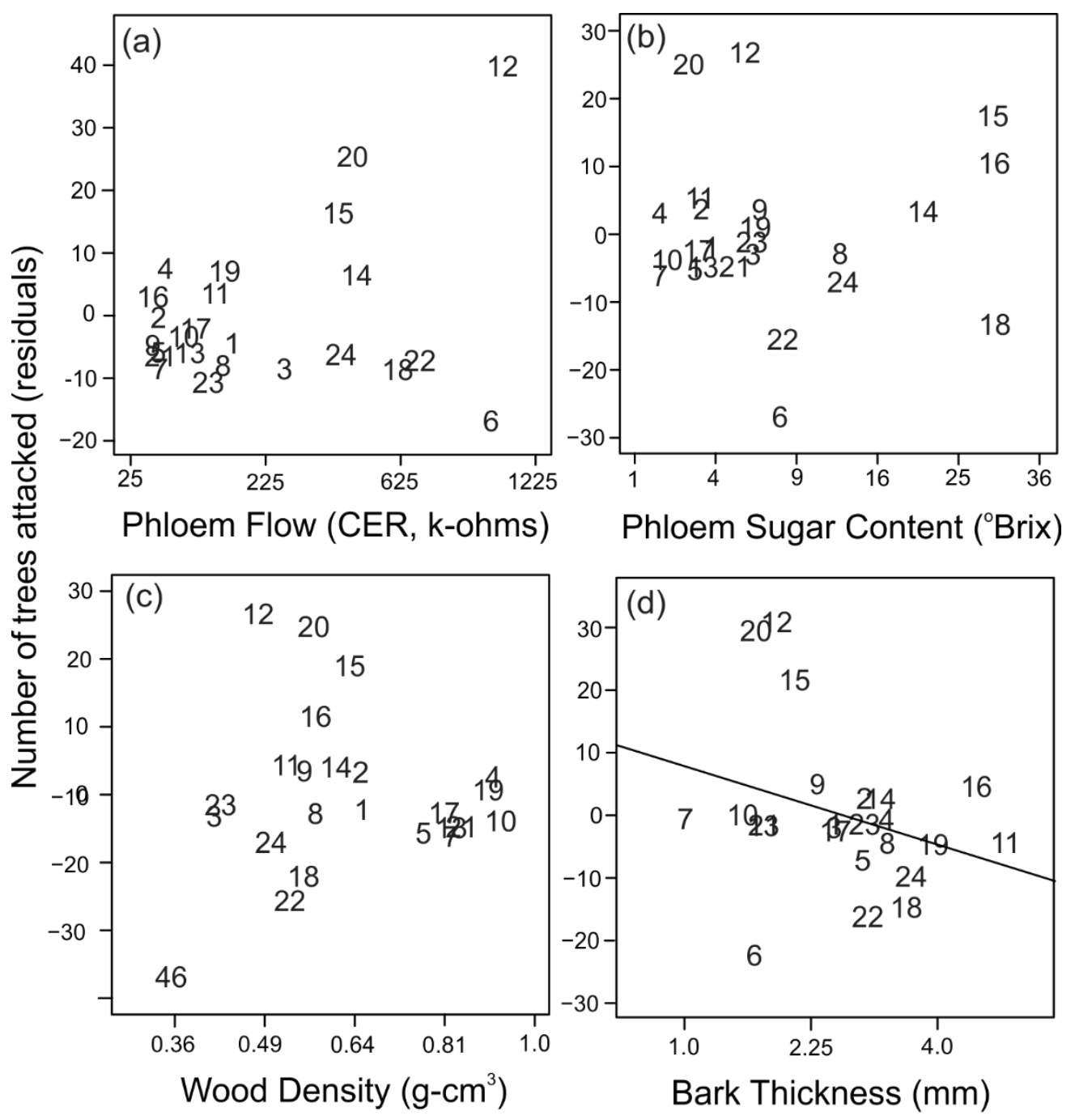

Tree Traits

Figure 3.5.1. Influence of tree traits on host specificity of Aenetus virescens larvae for the 24 most common tree species. (a) Phloem turnover (CER - khoms), (b) Phloem sugar content ( ${ }^{\circ}$ Brix), (c) Wood Density $\left(\mathrm{g}-\mathrm{cm}^{3}\right)$, (d) Bark Thickness (mm). All Y-axes show the residuals of the number of trees parasitised when the other three traits and tree size $(\mathrm{DBH})$ are accounted for. The numbered points represent the numbers assigned to each tree species specified in Table 3.1.

trees being evident. This implies unsuccessful attacks on non-hosts are just as likely to occur; however, evidence of such attacks were not detectable during this study. Whilst acknowledging that larvae may encounter non-host trees as often as host trees, I believe it is unlikely that unsuccessful attacks would pass undetected, 
primarily because of the behaviour of larvae when commencing the arboreal phase of their life cycle. When larvae leave the leaf litter and ascend a host tree, they first construct a webbing of loose silk strands over themselves on the outside of the tree before they start tunnel excavations (Yule \& Burns, unpubl.). This webbing protrudes from the tree, positioning the larvae directly between the webbing and the tree surface. This webbing is then bolstered with excavated wood pieces and incorporated into the final webbing that covers the tunnel entrance. To consider an attack unsuccessful, a larva would have to attempt an attack and then opt not to continue, leaving remnants of the "beginner" webbing behind. If larvae were making attempts to parasitise trees other than those defined here as hosts (Table 1), it is likely that evidence of these "beginner" webbings would have been found. An alternative explanation for the most abundant trees not being parasitised most often is that larvae are able to discriminate between heterogeneous tree species, seeking out or identifying hosts via stimuli.

Larvae appear to prefer particular host species, which were attacked significantly more than their abundance would suggest. For example, Aristotelia serrata was only present at Site 3, yet incurred one of the highest number of trees attacked (89\%), with only a few individuals not parasitised. Additionally, Carpodetus serratus was consistently one of the most heavily parasitised species across all three sites (Table 3 1). Both tree species appeared to be preferred hosts of larvae regardless of abundance; it is likely these are parasitised wherever present within the larvae's geographical range. Whilst larvae appear to discriminate between tree species, the stimuli used in host detection is currently unknown. One possibility is that larvae encounter several tree species at random that are rejected before a preferred host is found. An alternative explanation is that female moths oviposit eggs within the vicinity of preferred hosts, so larvae are already in close proximity to suitable hosts when commencing their search. However, larvae must still locate a suitable host after spending several months aggregated underneath fungi and leaf litter. Preferred trees are often riddled with larvae tunnels, whereas trees of non-host species-even when touching the preferred host tree-will not have a single larvae tunnel. Most Lepidopteran have evolved acute olfactory senses that respond to stimuli from plant signals or conspecific cueing to locate suitable hosts (Hansson 
1995). Olfactory cues are likely also important for the larvae, but which stimuli cue larvae towards hosts requires further investigation.

Phloem turnover and phloem sugar content, considered here to be larvae's reward from host trees, did not influence tree susceptibility to parasites. No relationship was found between either phloem turnover or phloem sugar content and the number of trees that were parasitised. One explanation is that phloem turnover relies heavily on environmental conditions such as rainfall, light, temperatures, rate of photosynthesis, reproductive cycle of hosts and other host sinks, most of which could not be controlled for in the field. This study presents a snapshot of phloem turnover and sugar content, and potentially did not capture the full variability of phloem flow rate and soluble sugars in trees. Another consideration is that larvae feed nocturnally, when temperatures are lower and photosynthesis is not occurring, so the movement of phloem is likely different from daytime samples (Douglas 2006), such as those used here. Furthermore, there is a close relationship between tree secondary metabolites and host colonization and establishment in bark beetles, which may also have influenced $A$. virescens larvae host use. Whilst investigating secondary metabolites of host trees were beyond the scope of this study, I recommend future work focuses on elucidating plant chemical signalling and defence and their relationship with host use in $A$. virescens. Despite these limitations, this study provides an interesting starting point that indicates that neither of the reward variables influenced the number of trees attacked by larvae. This suggests that something other than sugar rewards from host trees drives larval host specificity.

Whilst I considered phloem to be energetically rewarding owing to high sugar content and therefore carbohydrate availability to larvae, other nutritional qualities may be more important for larvae when using hosts. For example, the quantity and quality of phloem nitrogen and essential : non-essential amino acids vital for insect growth are relatively low (Douglas 2006). However, phloem feeders ingest enough nutrients for survival by breaking the sieve elements that transport phloem, allowing the consumption of large amounts of phloem sap, which includes all nutrients and proteins (Kehr 2006). Interestingly, phloem feeders often produce saliva that inhibits 
the production of callose, which heals broken sieve elements, instead keeping wounds open for continued feeding (Kehr 2006). As a result, trees with reduced healing abilities may be more important to larvae than nutritional quality of phloem, and warrants further investigation.

Hosts with reduced defensive traits were more susceptible to parasite attack. Bark thickness was the significant driver of larvae host specificity; thin-barked tree species were parasitised significantly more often than thick-barked tree species. Concurrently, bark thickness was a strong predictor of attack intensity in ponderosa pine Pinus ponderosa by the bark beetle Ips pini (Coleoptera: Curculionidae: Scolytinae) (Kolb et al. 2006). Yellow-bellied sapsuckers Sphyrapicus varius (Piciformes: Picidae) also used hosts with thinner bark for phloem feeding (Eberhardt 2000; Speights \& Conway 2010). Moreover, bark thickness, as a function of stem size, was a significant predictor of the spatial distribution of sooty beech scale insects Ultracoelostoma assimile (Homoptera: Margarodidae) on southern beech trees Fuscaspora spp. (Wardhaugh et al. 2006). Tree bark represents an external barrier that parasites must penetrate in order to reach the valuable cambium. In particular, larvae must get through the external bark to first construct a tunnel and, second, maintain a feeding scar around their tunnel entrance. As bark increases in thickness it is likely to become more energetically costly for phloem feeders to overcome, making thinner barked tree species more susceptible to attack.

In addition to being less energetically costly for larvae to overcome, thinner bark is indicative of smaller, faster growing tree species (King et al. 2006). For Lepidoptera, the size of the final instar ultimately determines adult fitness (Mega 2014). Larvae have $\sim 6$ years to achieve their maximum size of $100 \mathrm{~mm}$ before pupation. Larval growth is likely constrained by tree diameter because larvae can only grow within the limits of the tree. Concurrently, Aristotelia serrata, a preferred host of larvae, are one of the fastest growing tree species in New Zealand (Anton et al. 2015) and individuals as small as 1-cm DBH are often parasitised by larvae (Yule \& Burns 2014). Faster growing trees rapidly expand their trunk girth, allowing larvae to maximise their growth rate and reach optimal size sooner. Similarly, Barber (2005) found that parasites of fish grew larger in faster growing hosts. 
Wood density can also be considered a tree defence as harder wooded species are potentially more costly for phloem feeders, wood borers and xylem feeders to attack. Specifically, larvae need to successfully excavate and maintain a dwelling tunnel long term and I hypothesised wood density would likely be a significant tree defence against larvae parasitism. However, the results of this study indicated that wood density is not a significant driver of host specificity for larvae. Anecdotally, larvae are infamous for using Puriri trees Vitex lucens as hosts; these are one of the hardest wooded tree species in New Zealand. This supports the results that wood density is not a determinant of host specificity for larvae. Similarly, Feller and Mathis (1997) found no relationship between wood density and attack by wood-boring insects.

These results raise interesting questions regarding larvae host specificity in terms of optimal foraging theory (OFT). Contrary to these expectations, larvae are not parasitising host species that offer the greatest reward. However, a key assumption of OFT is that food items with the greatest energy rewards will be chosen more often, given an equal handling time. As this does not appear to be the case for larvae, it is likely that tree defences, such as bark thickness, negate "an equal handling time" because of the increased energy required to overcome thicker bark. Furthermore, wood-borers likely face significant selection pressures in the coevolutionary arms-race with their hosts. Theoretically, selection pressure acting upon circumventing host defences for a long-term parasite is perhaps greater than the selection pressures acting upon choosing hosts with maximum rewards. Ultimately, larvae can only benefit from trees with greater internal rewards if the trees' external defences can be overcome. Whether a trade-off exists for larvae between cost and reward of parasitising host trees should be of primary importance in future investigations.

Grehan (1984) observed a similarly broad range of larvae host species at alternative locations within New Zealand's lower North Island, also recording a 
considerable number of larvae in $A$. serrata and $C$. serratus individuals. This, along with the current study, supports the hypothesis that preferred hosts are attacked most often, despite multiple suitable hosts being available (Sears et al. 2012; Lootvoet et al. 2013). For generalist parasites exploiting multiple host species, the processes driving host specificity provides insights into parasite evolutionary ecology and their role in structuring ecosystems (Poulin 2007; Poulin et al. 2011). Consequently larvae may play a significant role in shaping local ecosystems. For example, larvae are a food source for threatened native birds such as kaka, which consume larvae in their arboreal phase, morepork Ninox novaeseelandiae (Strigiformes: Strigidae), which consume moths on the wing, and ground-dwelling ratites such as the little spotted Kiwi Apteryx owenii (Apterygiformes: Apterygidae), which are potential predators of leaf litter larvae. Additionally, when larvae emerge from their hosts as moths, empty tunnels are often used as refuges by forest invertebrates such as tree weta Hemideina spp. (Orthoptera: Anostostomatidae). Thus, host specificity of larvae has significant implications for the structuring of New Zealand forest ecosystems.

In this study, I presented the first investigation of the patterns and processes of host specificity for the larvae of New Zealand's largest endemic moth. I presented evidence that larvae can discriminate between tree species, consistently using preferred hosts with reduced defensive traits at different sites. These results suggest the pattern of host specificity for larvae can be explained by host defences but not by host nutritional rewards. Identifying the processes that make host trees susceptible to larvae provides a significant tool for addressing important patterns in larvae aggregation and virulence, and their role in shaping ecosystems. 


\section{Chapter 4. Non-adaptive camouflage: crypsis can obscure adaptive thermoregulation}

Adapted from:

Yule \& Burns (In Review) Non-adaptive camouflage: crypsis can obscure adaptive thermoregulation. 


\subsection{Abstract}

Camouflage in animals has traditionally been considered an anti-predator adaptation. However, the adaptive consequences of camouflage-for instance, increased survivability via predator avoidance-has rarely been tested. Here, I directly assess the adaptive consequences of crypsis on larvae survivability in New Zealand's largest endemic moth, Aenetus virescens (Lepidoptera: Hepialidae). Larvae live $\sim 6$ years as tunnel-dwelling arboreal parasites feeding on host tree phloem. Larvae construct a silk and frass webbing over their tunnel entrance that closely resembles the background bark. I hypothesised that webbing increases larvae survival by concealing them from predatory parrots, Nestor meridionalis (Psittaciformes: Nestoridae), who use powerful beaks to excavate larvae from inside host trees. I assessed whether webbing was visually cryptic to parrots via spectral analysis of webbing and background bark in avian tetrahedral colour space. Then, I ran a 26-month field experiment in which I manipulated webbing conspicuousness and directly assessed larvae survivability based on attacks of cryptic and conspicuous webbing. My results indicate webbing increased in visual crypsis across ontogeny. However, crypsis did not increase larvae survivability by reducing predation; cryptic webbing was attacked just as often and quickly as conspicuous webbing. As an alternative adaptive consequence of crypsis, I hypothesised that webbing would aid thermoregulation vital to insect growth. I assessed the temperature inside 50 larval tunnels both with and without webbing. Temperatures were significantly higher in tunnels with webbing compared with tunnels without. My results indicate that crypsis in prey did not protect them from predators. Instead, crypsis was likely a by-product of selection acting upon webbing properties that improved the living environment for larval growth. My study supports the newly emerging paradigm shift that indicates an organism's colouration may be more closely associated with abiotic conditions than biotic signalling. 


\subsection{Introduction}

Predation is one of the most significant selection pressures for organisms (Stevens \& Merilaita 2009). Co-evolutionary arms races between predators and prey are a foundation of natural selection and drive the evolution of traits and behaviour (Dawkins \& Krebs 1979). Animal colouration, patterns and behaviours have been discussed as an adaptive consequence of predator avoidance since the seminal works of Poulton (1890) and Thayer (1909). Despite camouflage being the most ubiquitous anti-predator defence strategy in animals (Poulton 1890; Cott 1940; Edmunds 1990; Ruxton et al. 2004; Troscianko et al. 2013), quantitative testing of the adaptive consequences of camouflage, i.e. whether predation is actually reduced, is scarce (Nylin et al 2001; Stevens \& Merilaita 2009; Troscianko et al 2013). Whether camouflage is directly an anti-predator adaptation or a non-adaptive consequence of selection acting on another primary function of colour (e.g. communication or thermoregulation (Nylin et al. 2001; Stuart-Fox \& Moussalli 2009)) is an unresolved interdisciplinary problem (Stevens \& Merilaita 2009; Troscianko et al. 2013; Stevens \& Tevens 2015) with quantitative studies emerging only in the last 15 years (Stevens \& Tevens 2015).

Whilst aposematic prey stand out and deter predators via colour signalling, cryptic prey have evolved colouration or markings that closely resemble their background (Edmunds 1990; Stuart-Fox \& Moussalli 2009). Crypsis encompasses an array of traits and behaviours exploiting visual concealment (reviewed in Stevens and Merilaita (2009); Stevens \& Tevens 2015). Being inconspicuous via mimicry is particularly common, especially amongst insects (Edmunds 1990); caterpillars of Oxytenis naemia mimic dead, rolled-up leaves (Nentwig 1985), stick insects (Phasmatidae) closely resemble twigs and bark and tenebrionid beetle Cossyphus resemble winged seeds (Cuthill et al. 2005; Hultgren \& Stachowicz 2008). Kelp crabs (Epialtidae) are not initially cryptic, but disguise themselves from predators by using items from their environment as decoration (Hultgren \& Stachowicz 2008). Conversely, early Saucrobotys instars change from cryptic to conspicuous across ontogeny, altering their colour signalling based on their physical defences (Grant 2007). 
The sensory system used by predators is a crucial component for successful camouflage. Birds in particular are visual predators; tetrachromatic vision allows distinction of a greater range of wavelengths than humans (Endler \& Mielke 2005; Stoddard \& Prum 2008). Visual predators are generally assumed to impose selection pressures on prey colouration (Forsman \& Appelquist 1998). For example, the diverse camouflage observed in insect prey, ranging from counter-shading to aposematism, is traditionally considered a form of anti-predator adaptation (Rowland et al. 2008). Likewise, some plant colouration is potentially adapted to avoid detection by avian predators (Fadzly et al. 2009; Schaefer \& Ruxton 2009). However, birds "make a living" identifying hard to see prey. Continuously encountering the same prey phenotypes presents learning opportunities for predators to identify cryptic prey more effectively (Edmunds 1990; Troscianko et al. 2013). The mechanisms driving camouflage, including how predators perceive prey and the resulting survival value inferred, remain unresolved in current understanding of defensive colouration (Stevens \& Merilaita 2009; Stevens \& Tevens 2015).

Here, I directly assess the adaptive consequences of ontogenetic crypsis, and its value in reducing predation, for the larvae of New Zealand's largest endemic moth. Aenetus virescens (Doubleday, 1843) (Lepidoptera: Hepialidae), hereafter "larvae", has arguably the most unique lepidopteran life history on Earth. Female moths (Fig. 4.2.1a) scatter eggs across the forest floor where first larval instars are mycophagous (Grehan 1987). After 3-4 months the larvae disperse, find their host tree, and excavate a "7"-shaped tunnel into tree heartwood. Larvae grow to 100 $\mathrm{mm}$, spending $\sim 6$ years as solitary tunnel-dwelling arboreal parasites (Grehan 1983; Tobi, Grehan \& Parker 1993), as shown in Fig. 4.2.1b. Feeding nocturnally on host tree phloem, larvae create large feeding scars around their tunnel entrance (Fig. 4.2.1c); they then construct silk and frass webbing over both the tunnel entrance and feeding scars (Fig. 4.2.1d). Older webbing looks much like the tree background, potentially concealing larvae from predatory parrots, Nestor meridionalis (Gmelin, 1788; Psittaciformes: Nestoridae, Fig. 4.2.1e), hereafter "parrot(s)", who consume $A$. virescens using powerful beaks to tear chunks of wood from trees (Fig. 4.2.1f). If 
webbing is damaged or removed, larvae reconstruct the webbing overnight. New webbing appears initially conspicuous, gradually becoming more like the background bark through webbing ontogeny. Potentially, larvae rely on cryptic webbing as a primary defence to conceal larvae activities from predators.
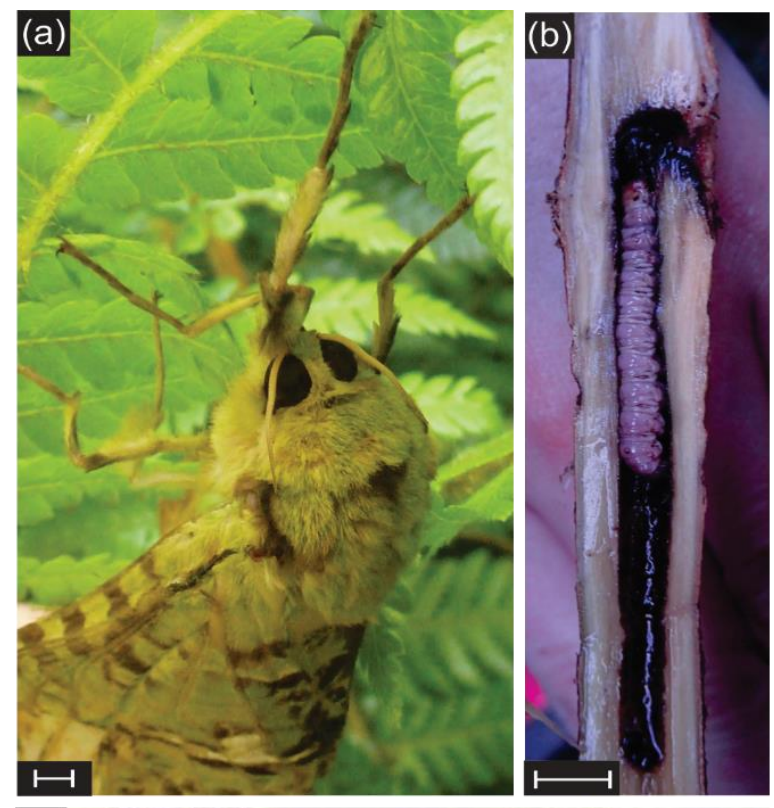

(d)

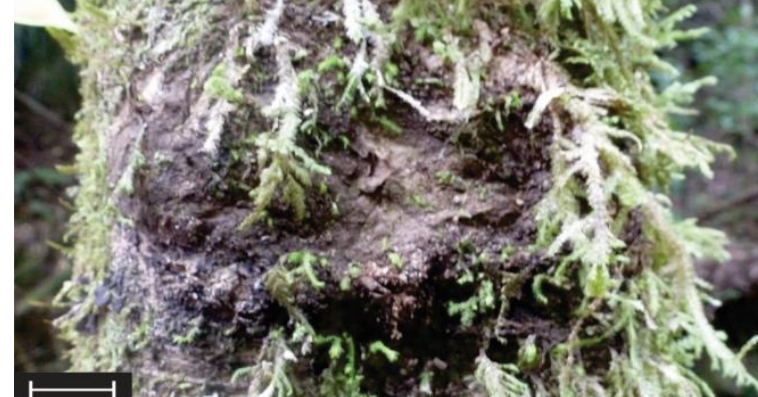

Figure 4.2.1. (a) Adult female Aenetus virescens, New Zealand's largest endemic moth. (b) A. virescens larvae in its self-excavated "7"-shaped tunnel inside a host

sapling. (c) Feeding scar with webbing freshly removed where $A$. virescens nocturnally extract phloem; larvae can be seen emerging from the tunnel entrance after the authors flooded the tunnel. (d) Webbing made from silk, frass and debris from the tree bark covers tunnel entrance. (e) Nestor meridionalis are the main predator of $A$. virescens and use powerful beaks to excavate bark and wood from trees to consume larvae. (f) The damage incurred by host trees after $N$. meridionalis excavate wood to consume $A$. virescens larvae. All scale bars represent $1 \mathrm{~cm}$. 
Here, I present the first investigation of the adaptive consequences of $A$. virescens webbing and directly assess whether crypsis reduces predation during a 26-month field experiment. Specifically, I posed the following three questions: i) is webbing visually cryptic to parrots? ii) if so, does cryptic webbing protect larvae from parrot attack? and iii) does webbing increase temperature inside larvae tunnels? and iv) does changes in temperature brought about by webbing affect larval growth rates?

\subsection{Materials and Methods}

\subsubsection{Study site}

Data were collected in Zealandia, a mainland island reserve at the southern tip of New Zealand's North Island $\left(41^{\circ} 28^{\prime} \mathrm{S}, 174^{\circ} 74 \mathrm{~W}\right)$. The climate is mild and temperate, with elevations ranging from 160 to 380 m.a.s.l. (Blick et al 2008). Primary forest was cleared for agriculture in the late 1800s (Blick et al. 2008; Burns 2013) and Zealandia comprises 225 hectares of successional broadleaf/conifer forest enclosed by a mammal-resistant fence. Introduced mammalian predators were eradicated in 2000, aiding the re-introduction of native fauna and flora, and in particular native birds (Burns 2013). Nestor meridionalis, a threatened parrot, was successfully re-introduced with $\sim 200$ individuals now resident, including regular breeding pairs. The forest comprises dominant broadleaf evergreen trees and a dense understorey includes tree ferns, shrubs and vines. Zealandia also boasts a well-established and abundant population of $A$. virescens larvae throughout the valley and surrounding hills, predominantly in preferred host Aristoteila serrata (J.R. Forster \& G. Forster; Elaeocarpaceae) (Yule \& Burns 2014).

\subsubsection{Experiment 1: Is webbing visually cryptic to parrots?}

Cryptic colouration is expected to be most effective when organisms are highly similar to their background. Using spectral analysis, I quantified the chromatic and achromatic similarities of background bark and larvae webbing at three ontogenetic stages. From A. serrata host trees, I collected webbing from 25 tunnel 
entrances ("old" webbing > 1 year old), ensuring webbing remained intact. Tree bark was collected from an area directly adjacent to each webbing. Within 24 hours of collection, three reflectance measurements were haphazardly taken from each webbing and bark piece in the lab using an USB Ocean Optics 2000 Spectroradiometer and Xenon Pulse X2 lamp Ocean Optics light source with diffuse Teflon-based white reflectance standard (see Fadzly et al 2009 for further methodology). As birds are unable to discriminate the far-red spectrum, I calculated spectra at 5-nm intervals between 300 to $700 \mathrm{~nm}$ with SpectraSuite software (Fadzly et al. 2009). For each removed webbing, larvae rebuilt an entirely new webbing overnight. To assess similarities of new webbing to background bark, I collected the re-built webbings within 7 days of the webbing being removed ("new" webbing $<7$ days). I also collected a piece of the feeding scar from each of our tunnels ("removed" webbing). Spectral analyses of new and removed webbing samples were conducted in the same way as old webbing.

I quantified whether webbing and bark were visually distinguishable from each other from a birds perspective in models of avian vision using "pavo" package (Maia \& Eliason 2013) in R v.3.1.1 statistical platform (R Core Team 2015). As the visual perception capabilities of $N$. meridionalis have not yet been quantified, I used the default average UV visual system; most bird species have ultraviolet sensitive cones (Endler \& Mielke 2005; Jones \& Siefferman 2014). I specified "forest shade" light conditions across all models to match the field site. Vismodel functions in pavo determined whether webbing and bark were distinguishable in avian tetrahedral colour space. Voloverlap attained the overlap of bark and webbing volumes in avian colour space and coldist calculated the just noticeable differences (JND) where delta $S(\Delta S)$ represents the difference in shape of the reflectance curve "chromatic", and delta $L(\Delta L)$ represents the difference in overall reflectance "achromatic" (Stoddard \& Stevens 2011; Jones \& Siefferman 2014). Using the standard threshold of 1.00 , where JND of 1.00 and below indicate two stimuli are indistinguishable, and JND greater than 1.00 show a continuum of more rapid discrimination between stimuli based on the avian visual system of our model (Siddiqi et al 2004; Stoddard \& Stevens 2011; Jones \& Siefferman 2014). 


\subsubsection{Experiment 2: Does cryptic webbing protect larvae from parrot attack?}

If crypsis is adaptive as is traditionally presumed, cryptic organisms should be discovered less quickly and have greater survivability than organisms that are conspicuous. Over the course of a 26-month field experiment, I manipulated the conspicuousness of webbing by changing the colour of the background bark surrounding the webbing ( $n=100)$. In total, I used five spray-paint treatments: light (cream) and dark (brown) backgrounds, both of which are within the natural colour spectrum of bark; a bright background that parrots would not normally experience on tree bark (yellow); webbing background not changed (control); and dark paint added then dabbed off so that although the colour remained natural, any confounding effect of the paint persisted (procedural control). Webbing were randomly assigned to one of our five categories and during bark colouration webbing were covered to ensure only the background bark was coloured. Colour patches were $\sim 3 \mathrm{~cm}$ wide for each webbing to ensure size of colour patch was not a factor in discovery or attack. All 82 webbing were monitored monthly for 26 months for signs of attack by parrots. Attacks were considered cavities excavated into the tree where larvae had been consumed (Fig. 4.2.1e). Background colour treatments were repeated every 6 months to avoid effects of fading over time. During the experiment, 18 larvae pupated and emerged from their tunnels and so were discounted from our analysis. "Survival" package in $\mathrm{R}$ was used to analyse the time until discovery for webbing in each treatment. A cox proportional hazard regression with tree as a random factor determined whether crypsis (control and procedural control) increased larvae survival compared with conspicuous treatments. Webbing size, webbing height on tree and tunnel aspect were not significant in the larvae survival model (all $P>0.10$ ) and so removed from the final analysis.

\subsubsection{Experiment 3: Does webbing increase temperature inside larvae tunnels?}

Thermoregulation is considered one of three primary functions of colour and pattern in animals (Nylin et al. 2001; Stuart-Fox \& Moussalli 2009). Lepidopteran larvae are ectothermic and rely heavily on environmental temperature for growth and 
development (Ruf \& Fiedler 2002). Thermoregulation was assessed as a potential adaptive advantage of webbing by investigating the air temperature inside 50 larval tunnels, both with and without webbing. Sampling days had sunny conditions with ambient air temperature of $17-21^{\circ} \mathrm{C}$. Tunnels experienced dappled sunlight throughout the day under forest shade conditions. Sampling took place during the late afternoon (between 1500 and 1900 hours) prior to the initiation of nocturnal feeding by larvae. Temperature inside tunnels with webbing, hereafter "present", were assessed first. A 1-mm diameter hole was made in the webbing centre and a wire temperature probe inserted into the tunnel. Temperature was recorded at two positions within each tunnel: at a point between 0 and $1 \mathrm{~cm}$ past the webbing, hereafter the "front", and at the furthest possible point inside the tunnel-between 4 and $8 \mathrm{~cm}$ depending on tunnel depth—hereafter the "back". As each webbing remained intact (larvae re-sealed our holes), each tunnel was sampled on 3 separate days, with at least 7 days in between sampling, and the mean recorded. Three further sampling days occurred under the same conditions but with webbing from the tunnel entrance removed (hereafter "removed") in the morning (between 0800 and 1100 hours) and temperatures recorded in the exposed tunnels between 1500 and 1900 hours on the same day. All outside temperatures were taken at the same time as tunnel sampling with the probe held $1-5 \mathrm{~cm}$ from the tree surface immediately in front of the tunnel. To assess the effect of webbing on tunnel temperatures, I ran a nested ANOVA with position of temperature recordings of the "front" or "back" nested within webbing "present" and "removed". Contrasts were assessed using a HSD Tukey post hoc analysis.

\subsubsection{Experiment 4: Does changes in temperature brought about by webbing affect larval growth rates?}

Webbing size is a proxy for larva size (Chapter 2). Prior to collecting tunnel temperatures, we calculated the annual growth of each larva over three consecutive years via the increase in webbing surface area. We photographed webbing annually $(n=50)$ and the surface area of each webbing in $\mathrm{cm} 2$ was calculated using digital analysis software ImageJ (Rasband, 2014). We ran a linear regression to test whether tunnel temperature affected larval growth using the mean annual growth of 
larva as our response variable and the average temperature inside tunnels with webbing present as our predictor variable. As larvae grow at a consistent rate throughout ontogeny (Yule and Burns, 2014), initial size of webbing was not included in our analysis.

\subsection{Results}

\subsubsection{Experiment 1: Is webbing visually cryptic to parrots?}

Webbing enhances crypsis across ontogeny, becoming less distinguishable to avian predators over time. Visual inspection of reflectance curves showed tunnel feeding scars (hereafter "removed" webbing) was dramatically different in colour "chromatic" and reflectance "achromatic" than background bark, with bark being much less varied and less reflective (Fig. 4.4.1a). Removed webbing had the highest mean chromatic (dS) and achromatic (dL) JND's (mean \pm SE: $d S: 24.24 \pm 1.47$ and $\mathrm{dL}: 30.02 \pm 2.68$, Fig. 4.4.1 $\mathrm{a}$ and b) making them highly discernible to avian predators. The new webbing reflectance curve was much more similar to background bark both chromatically and achromatically, with bark now being more reflective than webbing, Fig. 4.4.1c. New webbing had mean chromatic and achromatic JND's lower than that of removed webbing (dS: $18.32 \pm 4.95$ and dL: $19.43 \pm 4.08$, Fig. 4.4.1c and d), indicating that new webbing was harder to see for avian predators than tunnels with webbing removed. Old webbing reflectance curve was the most similar to background bark chromatically and achromatically, with background bark remaining more reflective than old webbing, Fig. 4.4.1e. Old webbing had the lowest mean chromatic and achromatic JND's (dS: $7.52 \pm 1.43$ and $\mathrm{dL}: 7.78 \pm 1.51$, Fig. 4.4.1e and f), indicating older webbing was much more difficult for avian predators to see than any other webbing stage. Further investigation revealed the chromatic JNDs were significantly different from each other in all three stages of webbing development (one-way ANOVA: $F_{3,73}=26.2, P<0.001$ ) with removed webbing and old webbing having the greatest significant contrast (HSD Tukey: Removed - New $=\mathrm{P}<0.01$, Removed - Old $=\mathrm{P}<0.001$, Old - New $=\mathrm{P}<$ 0.01). There was also a significant difference in achromatic JND's between webbing and background bark (nested ANOVA: $F_{3,73}=20.76, P<0.001$ ). However, whilst old 
webbing and new webbing had the greatest significant difference in achromatic JND, there was no significant difference in JND between removed webbing and new webbing (HSD Tukey: Removed - New $=P=0.13$, Removed - Old $=P<0.001$, Old - New $=\mathrm{P}<0.01$ ). Old webbing and the surrounding bark showed an overlap of $28.9 \%$ in our models of webbing and bark spectra volume in avian tetrahedral colour space, whilst new and removed webbing showed no overlap with their surrounding bark (Fig. 4.4.1b, $d$ and f). These findings support my hypothesis that older webbing is significantly more similar to its background, and visually more cryptic to parrots, than either new or removed webbing.

\subsubsection{Experiment 2: Does cryptic webbing protect larvae from parrots attack?}

There was no significant difference in the time it took for parrots to discover tunnels in any treatment $\left(X^{2}=0.6, d f=4, P=0.96\right.$, Fig. 4.4.2). A cox proportional hazard regression with tree as a random factor showed the risk of larvae being 


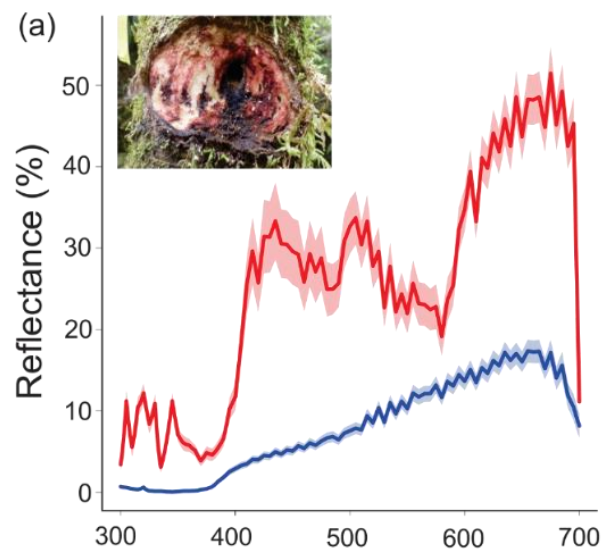

(b)
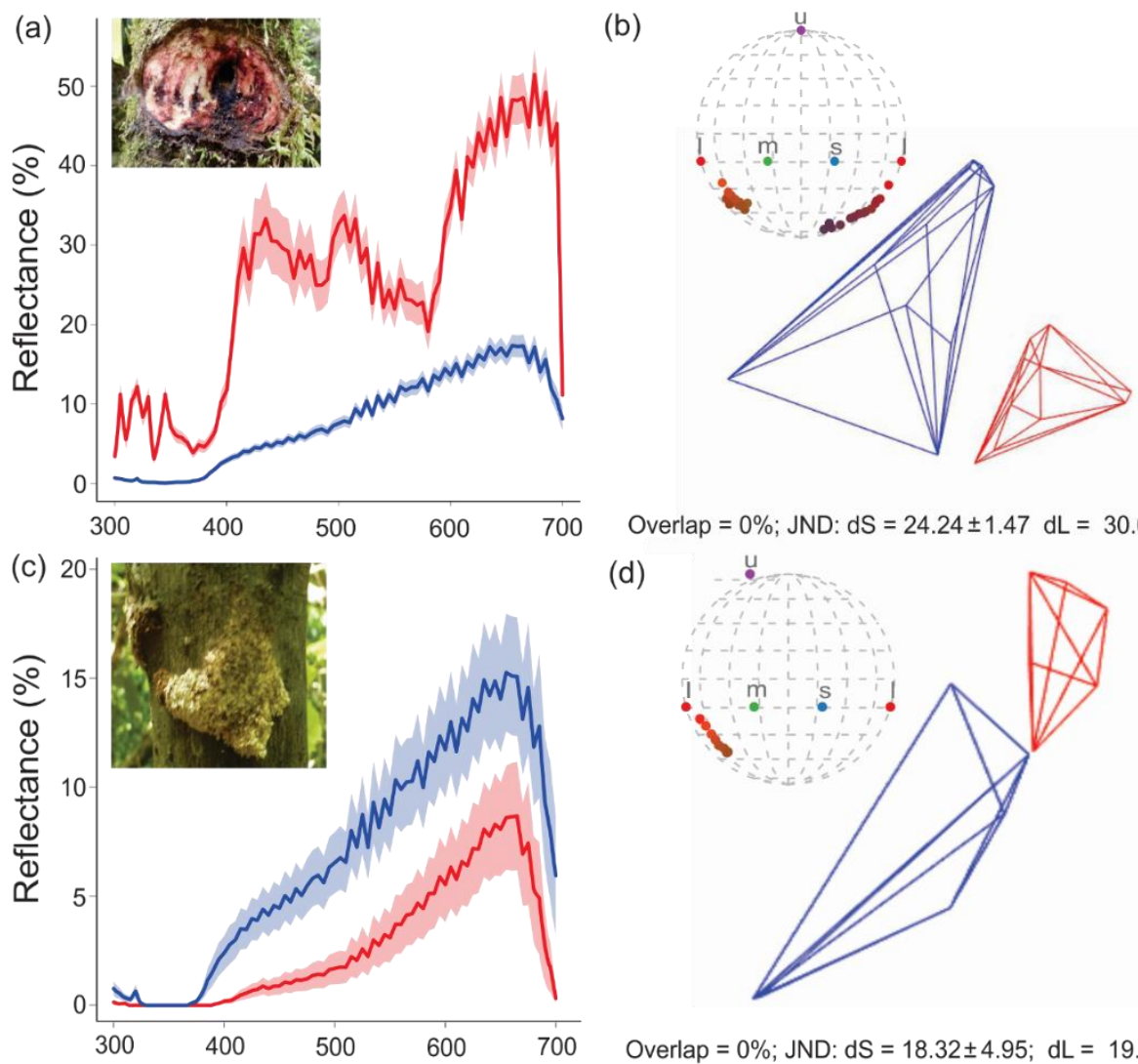

(d)
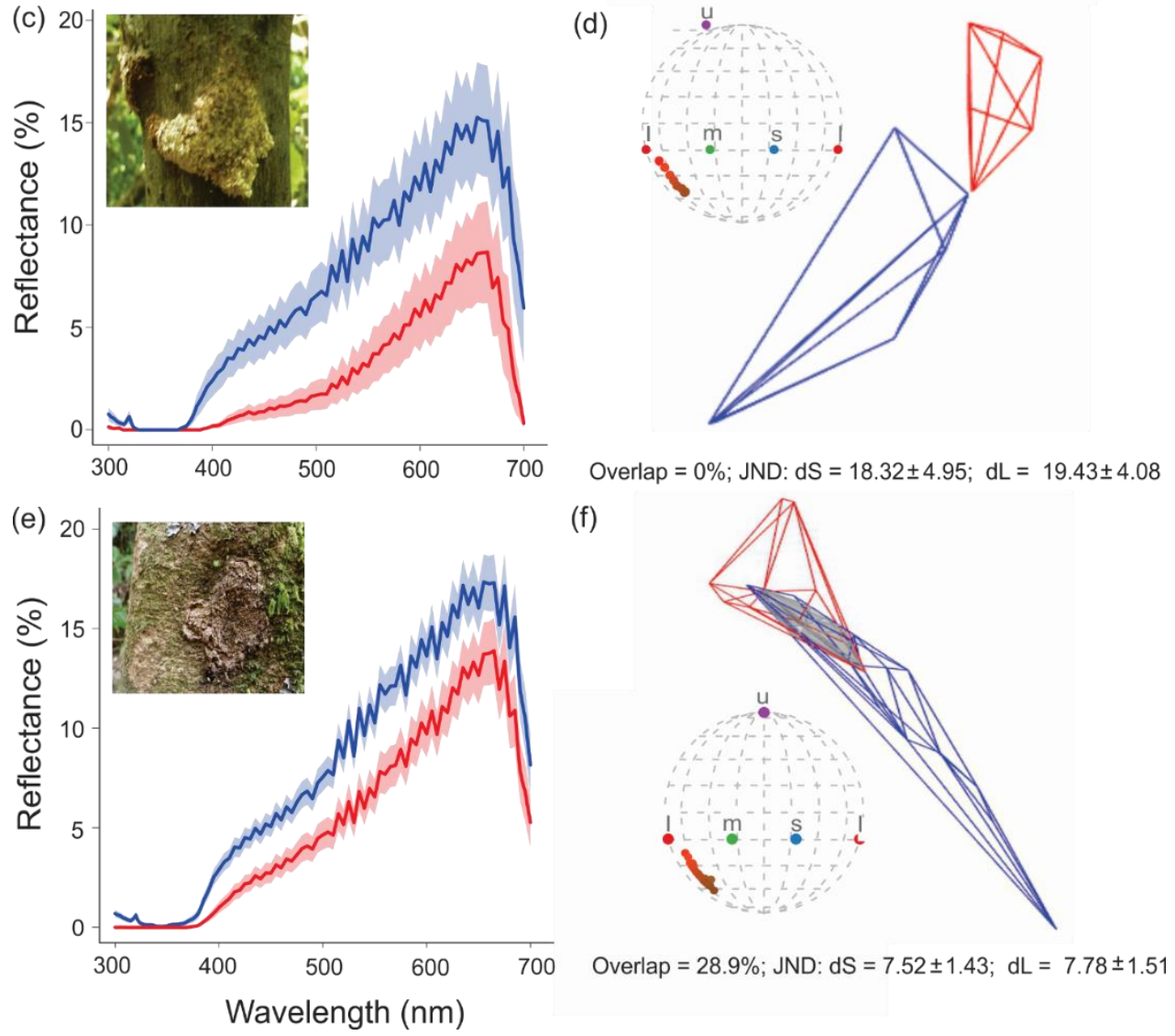

(f)

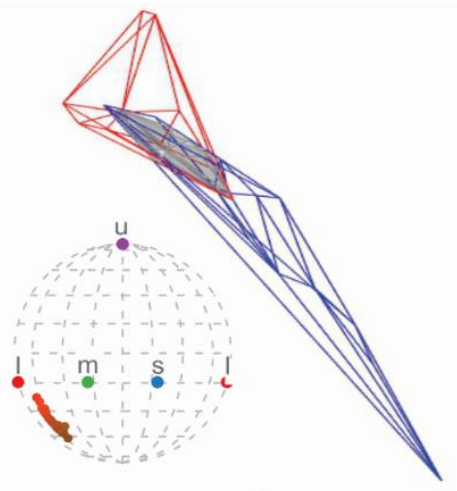

Overlap $=28.9 \% ; \mathrm{JND}: \mathrm{dS}=7.52 \pm 1.43 ; \mathrm{dL}=7.78 \pm 1.51$

Figure 4.4.1. Mean ( \pm SE, shaded areas) reflectance spectra for removed (a), new (c), and old (e) Aenetus virescens webbing (red) and the background bark surrounding webbing on host trees (blue). Volume taken up in avian colour space

between removed (b), new (d) and old (f) A. virescens webbing (red) and background bark (blue). Grey regions indicate an overlap under average UV and forest shade light conditions, with overlap \% and mean just noticeable differences (JND) for chromatic (dS) and achromatic (dL) \pm standard error detailed below each figure. Inserts in (b), (d) and (f) show bark and webbing as viewed in a projection plot of a tetrahedral avian colour space under average UV and forest-shade light conditions. Insert: $U, S, M$ \& $L$ refer to the wavelengths used in our models. $U=$ ultraviolet, $\mathrm{S}=$ short wavelength, $\mathrm{M}$ - medium wavelength, $\mathrm{L}$ - long wavelength. 
predated was statistically the same for all treatments (Fig. 4.4.2); the hazard ratio for risk of predation compared with our control was $0.95(95 \% \mathrm{Cl}=0.38$ to $2.34, \mathrm{P}>$ $0.10)$ for light background, $0.80(95 \% \mathrm{Cl}=0.18$ to $3.46, \mathrm{P}>0.10)$ for dark background, $0.70(95 \% \mathrm{Cl}=0.42$ to $1.16, \mathrm{P}>0.10)$ for bright background, and 0.98 $(95 \% \mathrm{Cl}=0.37$ to $2.55, \mathrm{P}>0.10$ ) for procedural control background. Cryptic webbing, which is significantly harder to see by avian predators, did not increase the survivability of larvae as they experienced the same level of attack and time until discovery as highly conspicuous webbing.

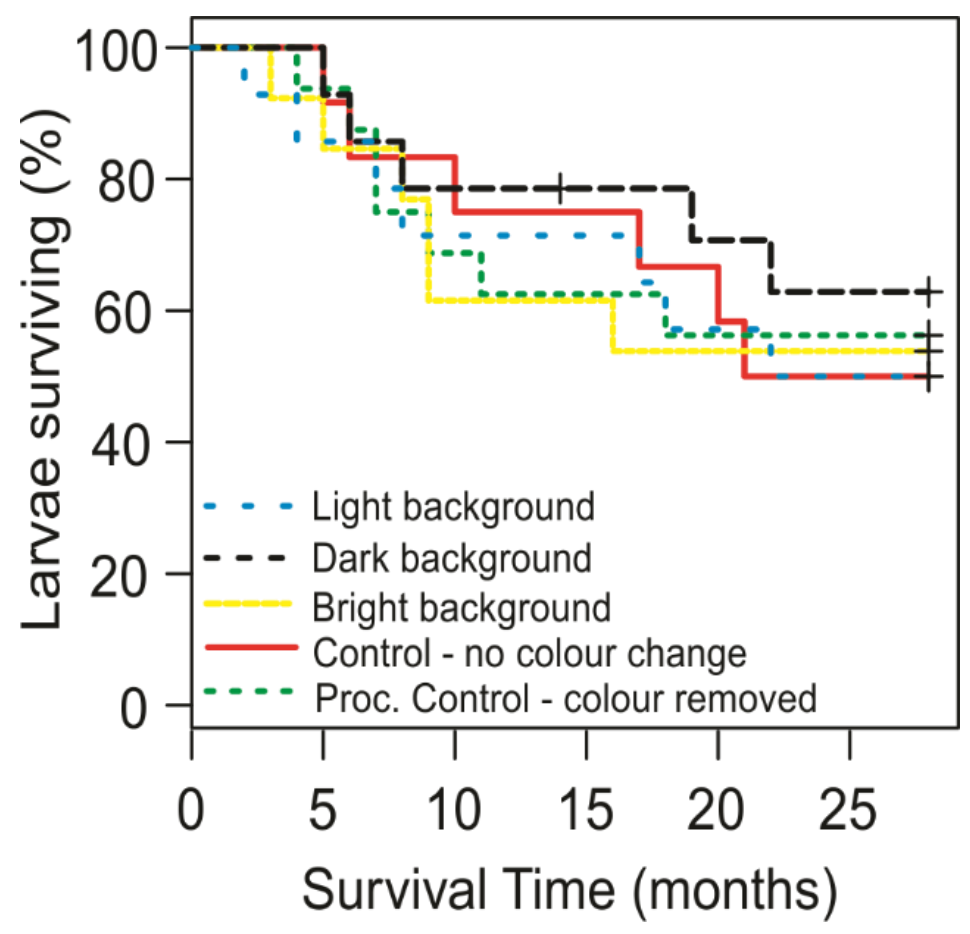

Figure 4.4.2. Survival of Aenetus virescens larvae over a 26-month field experiment in which the background colour surrounding their cryptic webbing was manipulated to create increased conspicuousness. Time until discovery and predation risk were statistically the same for all treatments, indicating cryptic webbing did not protect larvae from predation. 
A nested ANOVA revealed a significant difference in temperature inside tunnels with webbing "present" and tunnels with webbing "removed" $(F=149.5, \mathrm{df}=$ $1, P<0.001$, Fig. 4.4.3). Tunnels with webbing removed were significantly colder than tunnels with webbing present. The interaction of sampling positions front or back nested within the present or removed webbing was also significant $(F=134.1$, $d f=2, P<0.001$, Fig. 4.4.3). Contrasts were analysed with a post hoc HSD Tukey test which revealed a significant temperature difference between all conditions (all $P$ $<0.001$ except the contrast between "front: removed" and "back: present", which was still significant but at $P<0.05$, Fig. 4.4.3). Overall, temperatures at the furthest point from the tunnel entrance were lower than temperatures within the first $1 \mathrm{~cm}$ of the tunnel. All conditions showed a generally lower than ambient air temperature, except for the front of tunnels with webbing present, which had mostly higher than ambient air temperatures.

4.4.4. Does changes in temperature brought about by webbing affect larval growth rates?

Larvae grew more rapidly in tunnels with warmer temperatures $\left(r^{2}=0.43, t=\right.$ 6.02 , d.f. $=48, P<0.001$, Fig. 5). Webbing increased tunnel temperatures, facilitating more rapid larval growth. 


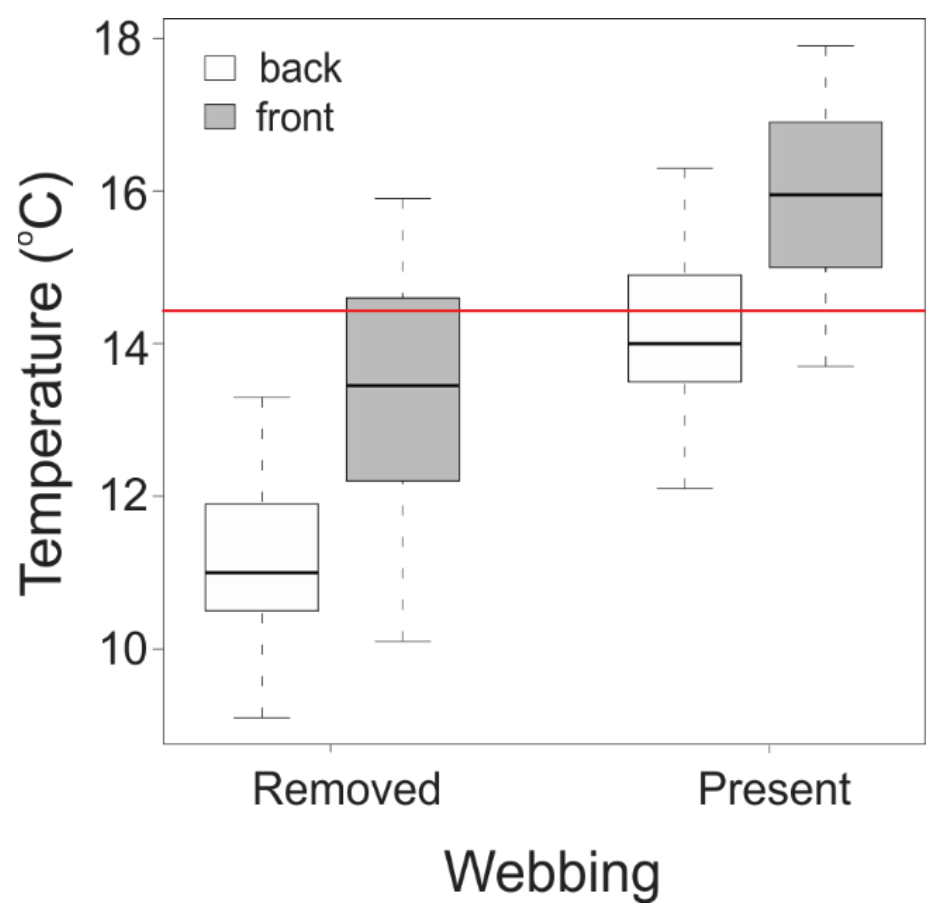

Figure 4.4.3. Effect of webbing (removed or present) and sampling position (Front: within $1 \mathrm{~cm}$ of webbing; Back: furthest point into tunnel, between 4 and $8 \mathrm{~cm}$ ) on temperature within Aenetus virescens larval tunnels. Red solid line represents the mean ambient outside temperature (within $5 \mathrm{~cm}$ of tree surface) for all sampling days. Contrasts were analysed using the HSD Tukey test after a nested ANOVA-all treatments were significantly different from each other $(P<0.001)$ and tunnels with webbing were significantly higher in temperature than tunnels with webbing removed $(P<0.001)$. 


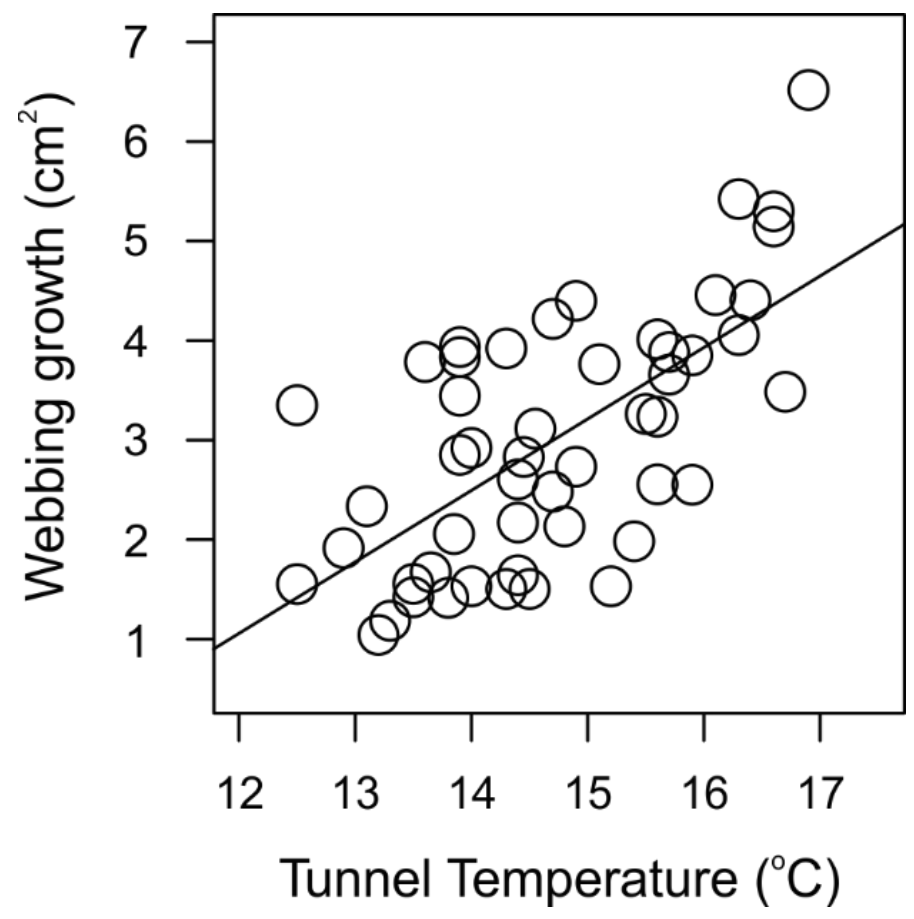

Figure 4.4.4. Relationship between the average tunnel temperature and the average annual growth of $A$. virescens larvae.

\subsection{Discussion}

In this chapter, the adaptive consequences of $A$. virescens webbing were examined and I directly tested whether crypsis reduced the predation of larvae. Results indicated that visual crypsis increased with webbing age. Old webbing ( $>1$ year) was significantly harder for parrots to see than new webbing ( $<7$ days) or tunnels which had webbing removed, exposing the feeding scar. The feeding scars where larvae consume host phloem consist of raw tree wounds and range in colour from pale cream to bright pink. Larvae cover these with webbing that is initially pale and conspicuous against the tree bark. Many organisms build structures or engage in decorating behaviour to increase their chance of avoiding detection by predators (Hultgren \& Stachowicz 2008). Similarly, larvae webbing is created from masticated wood pieces, frass and self-generated silk along with other materials from the environment. Over ontogeny the silk contracts and wood pieces deteriorate and 
decay, making the webbing a more compact and tightly woven structure, similar in appearance to that of the surrounding bark. Debris and epiphytes, such as moss and lichens, are often incorporated into the structure, enhancing the resemblance to the background. I observed three individual larvae construct tunnels adjacent to brightly coloured tagging tape that I placed during the study for tree identification, incorporating bite-size pieces of tape into their webbing. This suggests larvae are indiscriminately using whatever is directly around the feeding scar for webbing construction, regardless of conspicuousness. Should webbing be removed or damaged, larvae rebuild the webbing or fix damaged areas over one night, suggesting that web building is a priority that takes precedence even over feeding, because larvae cannot feed and build webbing simultaneously. Similarly, decorator crabs reduce feeding yet continue decorating in the presence of predators, indicating that potential anti-predator behaviour is more important than feeding for some prey species (Stachowicz et al 2012). Because webbing requires energy expenditure to construct, and appears to take precedence over feeding, the adaptive consequences of webbing are likely significant.

Despite webbing increasing in crypsis over ontogeny, webbing did not reduce attack by parrots. The results from a 26 -month field experiment indicated that despite being significantly harder to visually locate, cryptic webbing was not attacked less often than webbing that was made to be conspicuous. The time until discovery did not differ significantly between cryptic webbing and any of the conspicuous treatments, suggesting parrots are able to identify cryptic webbing just as quickly as conspicuous webbing. One possibility is that the conspicuousness treatments deterred parrots, resulting in no difference in attacks between cryptic and conspicuous webbing. However, these parrots are notorious for destructive behaviour, frequently pulling apart man-made structures (Charles \& Linklater 2013), and therefore conspicuous paint treatments were unlikely to deter attacks.

Potentially, parrots have learned to identify their highly cryptic prey through repeated encounters with the same phenotypes over time (Edmunds 1990; Troscianko et al. 2013). The relationship between predator and prey is a co- 
evolutionary arms race, a constant game of adaptation and counter-adaptation (Dawkins \& Krebs 1979). Although parrots are primarily visual predators, other sensory mechanisms, such as auditory cues, may be employed to locate visually cryptic prey-a theory the author is currently exploring. From an evolutionary perspective, predation is one of the most significant selection pressures an organism experiences and crypsis has traditionally been considered an adaptation to avoid detection (Stevens \& Merilaita 2009; Troscianko et al 2013). However, an organism that is visually cryptic but does not experience a reduction in predation suggests non-adaptive crypsis where alternative selection pressures result in a cryptic state incidentally.

Thermoregulation is considered a primary adaptive function of colouration in animals (Nylin et al. 2001; Stuart-Fox \& Moussalli 2009). Insects in particular rely on temperature for growth and development (Ruf \& Fiedler 2002). Tunnels with webbing had significantly higher temperature at both the front and back of tunnels compared with the same positions in tunnels without webbing. In particular, the first $1 \mathrm{~cm}$ of tunnel behind webbing was consistently higher in mean temperature than ambient temperature outside webbing. Temperatures cooled towards the back of tunnels but still remained higher in tunnels with webbing. Results indicate that larvae grew more rapidly in tunnels with higher temperatures. Webbing provides a "blanket" that facilitates a higher temperature within tunnels compared to external ambient air temperatures or tunnels with webbing removed. Interestingly, Levesque et al. (2002) observed reduced growth rates and lower consumption rates in forest tent caterpillars Malacosoma disstria (Lepidoptera: Lasiocampidae) at lower temperatures. Social Eriogaster lanestris (Lepidoptera: Lasiocampidae) build tents that maximise internal temperature whilst reducing direct solar radiation received from sun basking (Ruf \& Fiedler 2002). Along with aggregation and avoidance behaviours such as moving in and out of shaded or sunny areas, the tent structure is key to successful regulation of body temperature (Ruf \& Fiedler 2002). In the study system used in this paper, both new and old webbing were consistently less reflective than the background bark indicating webbing is better at absorbing light. These findings support my hypothesis that cryptic webbing aids thermoregulation in larvae. 
Aenetus virescens are solitary caterpillars, remaining entirely embedded for the duration of arboreal life stages. Larvae live away from direct sunlight, several centimetres deep inside the heartwood of a tree. Larvae are ectothermic with no means of controlling the tunnel environment itself and temperatures remain low within the tree. Webbing provides a "blanket" that increases temperature at the tunnel entrance and to a lesser extent towards the back of the tunnel where larvae remain throughout the day. The codling moth larvae, Cydia pomonella, prefer feeding and building larger cavities in areas higher in temperature and more radiated (Kührt et al. 2005). A. virescens larvae can potentially move between tunnel areas to maximise the warmer temperatures at the front, or remain in the depths of the tunnel if cooling is required.

The greatest temperature increase in tunnels is around feeding scars, potentially influencing the trees reaction to wounding. Enhanced healing of the feeding scar may occur if higher temperatures induce localised cambial reactivation and a general increase in cambial phenology (Begum et al 2008; Deslauriers et al 2008), leading to consistent, if not improved, food supply to larvae. Bark itself can absorb heat from solar radiation and cambial temperatures can differ significantly from that of ambient air temperatures, as shown in several European tree species (Nicolai 1986). However, trees with smooth thin bark, like that of Aristotelia serrata, showed little or no temperature difference between the surface and cambium (Nicolai 1986). Larvae are slow-growing, long-lived species and the nutritional value of phloem to insects, whilst rich in sugars, is generally poor in terms of nitrogen and essential : non-essential amino acids vital for insect growth (Douglas 2006). It is possible that increasing temperatures within the tunnel will enhance larval growth and development, with webbing providing the only tool for obtaining raised temperatures within the tunnel environment. Further work on larval body temperature and the internal thermoregulation of tunnels is required to fully elucidate the advantages of increased temperature induced by webbing structures. 
Animals are considered the primary biotic drivers of colour evolution in prey. Here, I presented the adaptive consequences of $A$. virescens webbing, and explored whether reduction in predation was achieved via increasing crypsis across ontogeny. Results indicated that crypsis did not protect larvae from predatory parrots. Instead, crypsis was likely a by-product of selection acting upon webbing properties that improved the living environment for larval growth. This study supports an increasing body of literature that indicates an organism's colouration may be more closely associated with abiotic conditions than biotic signalling (Burns 2015). Further quantitative studies of prey survivability are needed to elucidate the true adaptive consequences of colour and patterns in animals. 


\section{Chapter 5. A unified explanation for male-biased susceptibility in animals and plants}

Adapted from:

Yule, K and Burns, KC (In Preparation) A unified explanation for male-biased susceptibility in animals and plants. 


\subsection{Abstract}

Males are often the "sicker sex", harbouring higher pathogen and parasite loads than females. Primarily, males invest in secondary sexual traits-a trade-off that weakens their immune system, increasing susceptibility. In animals, male-biased susceptibility (MBS) has received considerable attention, and emerging evidence indicates that MBS also occurs in plants; plants, however, lack an analogous immunosuppressing trade-off. Here, for the first time, I formally amalgamated MBS research for animals and plants by compiling the largest database of host-parasite species pairings to date $(n=461)$. Then, I ran a meta-analysis to evaluate the degree of MBS amongst individual host species and families. Next, I developed a new theoretical model to explain MBS in animals and plants, hypothesising that in species where females retain and nourish offspring post syngamy, parasites compete with offspring for female resources. The model was tested using independent, empirical data from a dioecious host tree-parasitic larvae system. Finally, I tested for differential resistance, i.e. plant "immunity" between male and female host trees as an alternative explanation for MBS.

Overall, 188 studies were included in the meta-analysis, comprising 409 hostparasite pairings from 70 animal families, and 52 host-parasite pairings from 22 plant families. MBS was significantly higher for both animal and plant families than would be expected by chance. Results from empirical testing of the theoretical model supported the hypothesis that male-biased susceptibility is a result of parasites competing with offspring for female resources. Thus, I provide the first explanation for MBS in my study system, and present a theoretical model which can potentially be applied to other host parasite systems where females retain and nourish offspring post-syngamy. 


\subsection{Introduction}

Parasites are ubiquitous, yet the complex interactions between parasites and their hosts often limit the generalisations that can be made regarding specific host or parasite species (Poulin \& Forbes 2011). Only recently have parasites been recognised as a driving force in ecology and evolution (Zuk \& Stoehr 2002), potentially altering host life cycles and population dynamics (Poulin 2007), and even influencing the evolution of their hosts' sexually selected traits (McCurdy et al. 1998). In turn, parasites rely solely on their hosts for nutrition, shelter and ultimately fitness (Tscharntke 1992; Lafferty et al. 2008; Johnson et al. 2010; Goedknegt et al. 2012; Friman \& Buckling 2013). Hosts vary dramatically in their characteristics, particularly between sexes, yet only recently has host sex been considered an underlying mechanism of susceptibility to parasites (Goble \& Konopka 1973; Alexander \& Stimson 1988; Bundy 1988). Subsequently, differences in parasite prevalence and infection status between conspecific male and female hosts have frequently been reported, emphasising differences in parasitological parameters between host sexes (Soliman et al. 2001; Krasnov et al. 2005b). However, the mechanisms underpinning the differences driving sex-biased susceptibility in hosts remain poorly understood.

In general, a precedence for male-biased susceptibility (MBS) to parasitism has been proposed by myriad studies published in the last 50 years (see Appendix Table A.2.). Overall, both endo- (Poulin 1996) and ecto-parasites (Morand et al. 2004) show bias for male hosts. Among human and non-human animals, for example, the prevalence and intensity of parasitic infection is higher in males than females (reviewed in Klein 2004). Birds and mammals in particular appear to be significantly male-biased in parasite infestations (Krasnov et al. 2005b). In animals that express sexual size dimorphism (SSD), males are often larger than conspecific females, making them a larger target with potentially greater resources for parasites (Klein 2004). Moreover, males often have a higher chance of exposure to parasites owing to their greater dispersal, aggression and aggregation behaviours in comparison with females, potentially increasing male exposure and contact time to both endo- and ectoparasites (Klein 2004). The primary explanation for MBS in animals, however, is the difference in immuno-competence between males and 
females based on androgens suppressing the immune system in males (Zuk \& McKean 1996; Zuk \& Stoehr 2002; Krasnov et al. 2005b). This key difference between sexes is the most commonly cited explanation for the pattern of MBS in animals (see references in Appendix Table A.2.). However, direct tests of this theory are scarce and the differences in the androgen-immune system between male and female hosts is not well understood (Klein 2004).

For decades, the pattern of male-biased susceptibility (MBS) to parasitism has held precedence in the animal literature and, until recently, the pattern of MBS in plants has been overlooked. Although uncommon on a global scale, an estimated $7 \%$ of plant genera are dioecious, where male or female functions occur in separate plants (Barrett 2002, Kavanagh 2011). Ashman (2002) suggested dioecy has evolved in more than half of all plant families. One of the key drivers of the evolution of dioecy from a hermaphroditic life style is the selection pressure of herbivores and natural enemies (Ågren et al. 1999; Ashman 2002; Cornelissen \& Stiling 2005a). Herbivores remove material and nutrients from their host plant, often with no reciprocal gain for the host. In these terms, herbivores can be considered parasites sensu lato, and will be amalgamated with parasites sensu stricto for the remainder of this chapter.

An emerging body of evidence suggests a similar pattern of MBS for males and females of dioecious plants (Agren 1999). Differential allocation to reproduction, with females investing more heavily than males (Feller 2002; Cepeda-Cornejo \& Dirzo 2010), phenology (Boecklen et al. 1990), nutritional quality (Hjältén 1992; Uribe-Mú \& Quesada 2006a) and defence characteristics (Tsuji \& Sota 2010) have all been proposed to result in differences in parasite intensity or prevalence between plant sexes (reviewed in Ågren et al. 1999 and Cornelissen and Stiling 2005). Unlike animals, plants do not have an analogous androgen-immune system trade-off resulting in immunosuppression. Concurrently, arthropod hosts also lack any system which parallels the androgen-immune system with Zuk \& McKean (1996) arguing that "...there is no parallel to the hormone testosterone simultaneously suppressing the immune response and facilitating sexual traits, making it less likely that selection 
would produce the same relationship between susceptibility and sexual development that exists in vertebrates..." (Wedekind \& Jakobsen 1998). The immunosuppression caused by testosterone in male vertebrates has no parallel in plants, however, there may be differences in plant resistance, i.e. plant "immunity" between sexes (Williams et al. 2011).

Parasites present a significant conflict in the battle for host resources in both male and female hosts (Hurd 2001). However, offspring, by nature, also take resources from females. Whilst female investment in offspring results from maternal adaptations to increase their own Darwinian fitness, ultimately offspring syphon female resources, competing directly with mother and siblings (Shaanker et al. 1988). Seeds often show a negative correlation between, for example, grain number and stem mass, demonstrating genetic conflict between parents and offspring (Sadras \& Denison 2009). Furthermore, seed mass variability has been directly related to the number of seeds sharing a fruit with individual seed mass decreasing as number of seeds per fruit increases (Banuelos \& Obeso 2003). In animals, conflict for maternal resources is manifested via sibling rivalry. For mammals that produce multiple offspring, an increasing number of offspring per litter results in smaller individuals that grow more slowly than in litters with less individuals (Hudson \& Trillmich 2008). For example, in Galapagos fur seals Arctocephalus galapagoensis (Carnivora: Otariidae) and sea lions Zalophus wollebaki (Carnivora: Otariidae), a mother still nursing a dependent offspring from the previous years' breeding season, produced offspring who were significantly disadvantaged in utero, being born smaller and growing slower than offspring to mothers who had no concurrent dependent offspring (Trillmich \& Wolf 2008). Evidence suggests mothers with increasing numbers of dependent offspring are less able to provide compensatory resources for the greater number of offspring (Hudson \& Trillmich 2008). This conflict between offspring and mothers, and between siblings, indicates an intense competition for the finite resources of females that has no parallel in males. Thus, females of animals and plants represent an already depleted pool of resources for parasites.

Conversely, male hosts without the burden of offspring represent an approximately full resource pool, potentially able to support a greater number of parasites than females. 
As a result of evolutionary isolation on islands, some geographic regions, such as New Zealand and Hawaii, have a particularly high incidence of dioecy (Kavanagh et al., 2011). In New Zealand, dioecy has been recorded for $\sim 18 \%$ of plant genera. Aristotelia serrata (J.R.Forst. \& G.Forst.; Eleaocarpaceae), a fastgrowing dioecious tree is the preferred host of New Zealand's largest endemic moth, Aenetus virescens (Doubleday, 1843) (Lepidoptera: Hepialidae), a long-lived arboreal parasite (hereafter, larvae). Larvae and $A$. serrata host trees have a remarkable association; larvae spend 3-4 months as mycophages in the leaf litter (Grehan 1987) before selecting a host tree and excavating a "7"-shaped tunnel into tree heartwood. Larvae grow to $\sim 100 \mathrm{~mm}$, living $\sim 6$ years as solitary tunnel-dwelling parasites (Grehan 1983; Tobi, Grehan \& Parker 1993). Feeding nocturnally on host tree phloem, larvae create large feeding scars around their tunnel entrance. Larvae construct silk and frass webbing over the tunnel entrance, potentially concealing themselves from predatory parrots Nestor meridionalis (Gmelin, 1788)

(Psittaciformes: Nestoridae) who consume $A$. virescens, using powerful beaks to tear chunks of wood from trees. Despite this remarkable association with $A$. serrata host trees, no information exists on whether a differential use of host sex exists for $A$. virescens.

Here, for the first time, I amalgamated MBS research for both animals and plants. Specifically, I aimed to: i) determine the magnitude of MBS for both animals and plants by compiling the largest database of host-parasite pairings to date ( $n=$ 461) from 188 studies; ii) present a new, unified theoretical model to explain MBS for both animals and plants, hypothesising that parasites compete with offspring for female resources; iii) test the predictions of the theoretical model using empirical data from the dioecious host tree $A$. serrata-parasitic larvae system; and iv) test for differential resistance, i.e. plant "immunity" between sexes of host trees as an alternative explanation for MBS. 


\subsection{Methods}

\subsubsection{Meta-analysis}

To assess if the pattern of MBS was significant in both animals and plants I first identified the key reviews of sex-biased parasitism or herbivory from the past 30 years. I identified eight separate reviews that focused on small mammals (Krasnov et al. 2012), arthropods (Sheridan et al. 2000), vertebrates (Klein 2004), birds (McCurdy et al. 1998) and plants (Ågren et al. 1999; Cornelissen \& Stiling 2005), and general reviews of MBS by Poulin (1996) and Moore \& Wilson (2002). From these reviews, I located the individual manuscripts used and extracted from each the host species inspected, the male and female host sample sizes if stated, each parasite species recorded for each host species, the prevalence or intensity of parasites per host species and the overall explanation the authors gave for the patterns they identified. I then searched the literature, using Google Scholar and the search engine of Victoria University of Wellington's online library, for the terms "sex-biased parasitism", "gender-biased parasitism", "sex-biased herbivory", and "gender-biased herbivory" published between 1996-2016 to supplement the reviews with manuscripts dated after their publication.

To be included in the meta-analysis, studies had to provide results of a statistical analysis comparing either parasite prevalence or parasite intensity between host sexes. Not all studies included set out to test susceptibility between sexes; however, many reported it as a descriptive addition and these were also included in the meta-analysis. I excluded studies that focused solely on juvenile individuals or that did not separate juveniles from adults for analysis. Studies that investigated natural or experimental infections were included, but I excluded all but the control groups from experiments where inoculations occurred that manually manipulated parasite load. In total, 188 studies (156 animal; 32 plant) contributed to the meta-analysis. 
To test for an overarching pattern in MBS for both animals and plants, I ran Bernoulli trials to assess the likelihood of the observed bias from the meta-analysis occurring at random. I used the sum of host-parasite pairings that were reported to be male-biased, the total number of host-parasite pairings and specified the expected probability of being male-biased by chance at $50 \%(0.5)$. However, owing to the over-representation of many host species being from the same taxonomic families, these tests were greatly overpowered. To account for the large phylogenetic component in the meta-analysis, I grouped host species by family and calculated the mean male bias observed for each family. To calculate the expected means for each animal and plant family if bias was occurring at random I ran computer simulations that randomly extracted either 0's (female bias) or 1's (male bias) for a comparable number of replicates per family. I repeated each simulation 1000 times per family and extracted the mean. I then compared my observed mean bias with expected mean bias across the family groups for both animals and plants using WilcoxonMann-Whitney non-parametric tests. To assess if animals and plants differed from each other in patterns of susceptibility, I ran a Wilcoxon-Mann-Whitney nonparametric test on the observed mean bias for animals and plant families.

\subsubsection{Theoretical model}

Based on the results of the meta-analysis, I hypothesised that differences in host susceptibility between the sexes results from parasite-offspring competition in females. Here, I develop theoretical model to explain male-biased parasitism in animals and plants.

\subsubsection{Model Parameters}

Let $P$ equal the number of parasites and $S$ equal the number of seeds. Based on the conceptual framework of Lotka Volterra population growth models and MacArthur's competition coefficient, let $\propto_{P S}$ equal the coefficient equating the energetic cost of seeds to the energetic cost of parasites, assuming 1) pre-syngamy, reproductive costs are the same for males and females, and 2) parasites are not attacking offspring. 
Then,

$$
P_{\overbrace{}^{\top}}=P_{+}+\propto_{P S} S
$$

where the number of parasites in male hosts $\left(P_{\sigma^{\prime}}\right)$ is equal to the number of parasites in female hosts $\left(P_{\varphi}\right)$ plus the number of parasites equivalent to the energetic cost of a seed $\left(\propto_{P S}\right)$ multiplied by the number of seeds $(S)$.

\subsubsection{Empirical Test}

I used the parasitic $A$. virescens - $A$. serrata host tree system to empirically test the predictions of my theoretical model. First, I tested whether $P_{\sigma^{7}}>P_{\varphi}$. From Zealandia, a predator free sanctuary in the Wellington region of New Zealand's North Island

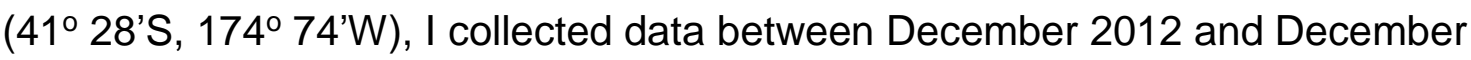
2015 from a randomly selected subset of male and female $A$. serrata trees (hereafter "host"). Zealandia consists of mixed broadleaf-podocarp forest (Yule \& Burns, 2014) and has an established host population with an abundant infestation of $A$. virescens (hereafter "larvae"). Larvae and hosts are found only at lower altitudes therefore data were collected within valleys and surrounding hillsides ranging from $160 \mathrm{~m}-500 \mathrm{~m}$ above sea level. The area receives annual rainfall for the region averaging $1200 \mathrm{~mm}$, with annual temperatures averaging $13.2^{\circ} \mathrm{C}$ (Macara 2014).

I identified the sex of hosts by the presence (female) or absence (male) of fruits. In total, I assessed 20 male trees with parasites, 20 female trees with parasites, and 20 female trees without parasites. Trees were assessed each fruiting season for three years to ensure trees were accurately sexed and accounting for annual variation in seed fruit production. To ensure mature, reproductive adults were assessed, only trees $>6 \mathrm{~cm}$ diameter at breast height $(D=1.25 \mathrm{~m})$ were included in the study. For each tree I recorded $D$, the number of larvae present, and the number of fruits for each female for three consecutive annual fruiting seasons. The number of fruits were calculated by taking digital photographs of 5 racemes per tree. Using digital imaging software Image $\mathrm{J}$ (Rasband 2014), digital counts of the number of fruits were obtained for each raceme and the mean fruits per raceme were calculated. As each 
fruit produces 8 seeds, I multiplied the number of fruits by 8 , then multiplied the result by the average number of racemes per tree for the three years.

A generalised linear model with poisson distribution confirmed that $P_{\sigma^{\top}}$ was significantly higher than $P_{q}(\mathrm{Im}: z=10.64, \mathrm{df}=37, \mathrm{P}<0.001$, Figure 5.3.1.). Trees grow continuously and diameter at breast height $(D=1.25 \mathrm{~m})$ was a significant covariate of larvae load ( $\mathrm{Im}: \mathrm{z}=6.586, \mathrm{df}=37, \mathrm{P}<0.001$, Figure 5.3.1.) and was therefore accounted for in our model.

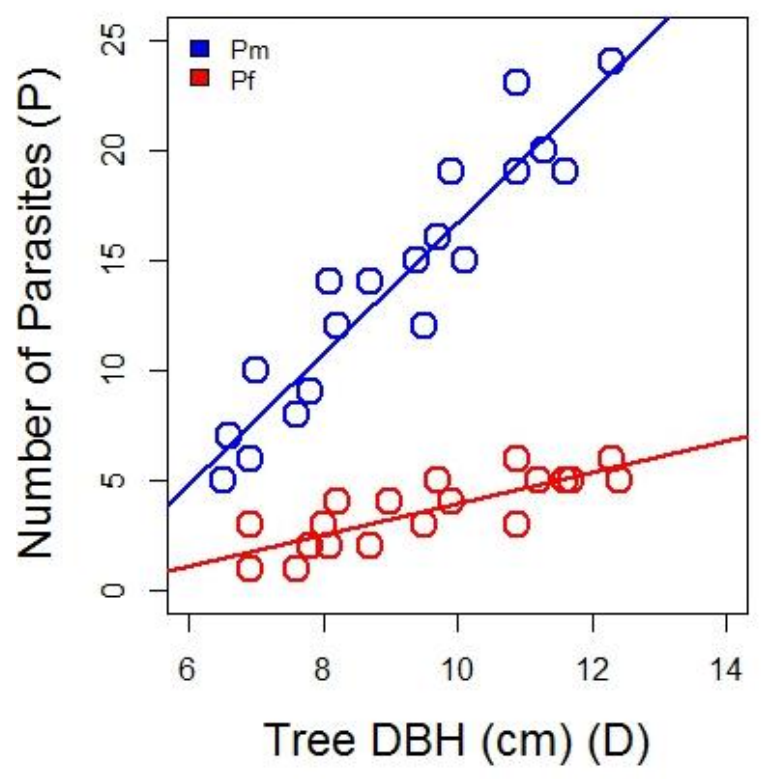

Figure 5.3.1. The relationship between the number of parasites on male hosts $\left(P{ }^{\top}=\right.$ $\mathrm{Pm}_{\mathrm{m}}$ and tree diameter at breast height $(D=1.25 \mathrm{~m})$ (blue), and the number of parasites on female hosts $\left(P_{\uparrow}=\mathrm{P}_{\mathrm{f}}\right)$ and $D($ red $)$.

To derive $\propto_{P S}$, I used $D$, the common factor between both male and female hosts, to scale the energetic cost of seeds with the energetic costs of larvae (Figure 5.3.2a and $\mathrm{b}$ ). I performed two linear regressions where $D$ was the predictor variable with the response variable being either the number of seeds for unparasitised female trees (female), or the number of parasites for male trees (male). Let $m$ equal the 
slope and $b$ equal the intercept from the linear regressions. Then, for unparasitised female trees

$$
S=m D+b
$$

where the number of seeds $(S)$ is equal to the slope of the regression $(m)$ multiplied by $D$ and adding the intercept (b) (Figure 5.3.2a). I used the same equation for male trees,

$$
P=m D+b
$$

but for the number of parasites $(P)$ (Figure 5.3.2b).

To solve for $D$, thereby scaling seeds to parasites via $D$, I rearranged the equation so that

$$
D=\frac{S-b q}{m q}=\frac{P-b \sigma^{\pi}}{m \sigma^{\pi}}
$$

where $D$ is equal to the number of seeds $(S)$ minus the female intercept $\left(b_{+}\right)$, divided by the female slope $\left(m_{\uparrow}\right)$ This is also equivalent to the number of parasites $(P)$ minus the male intercept $\left(b_{3}\right)$, divided by the male slope $\left(m_{3}\right)$.

Thus, to derive the coefficient that equates the energetic cost of seeds to the energetic cost of parasites $\left(\propto_{P S}\right)$, the equation for $D$ is rearranged to calculate $P$

$$
P=m \sigma^{\prime}\left(\frac{S-b q}{m q}\right)+b \sigma^{\prime}
$$

Where the number of parasites $(P)$ is equal to the male slope $\left(m^{3}\right)$ multiplied by the whole calculation for the number of seeds $(S)$ minus the female intercept $\left(b_{\odot}\right)$ and divided by the female slope $\left(m_{+}\right)$, plus the male intercept $\left(b_{3}\right)$. 

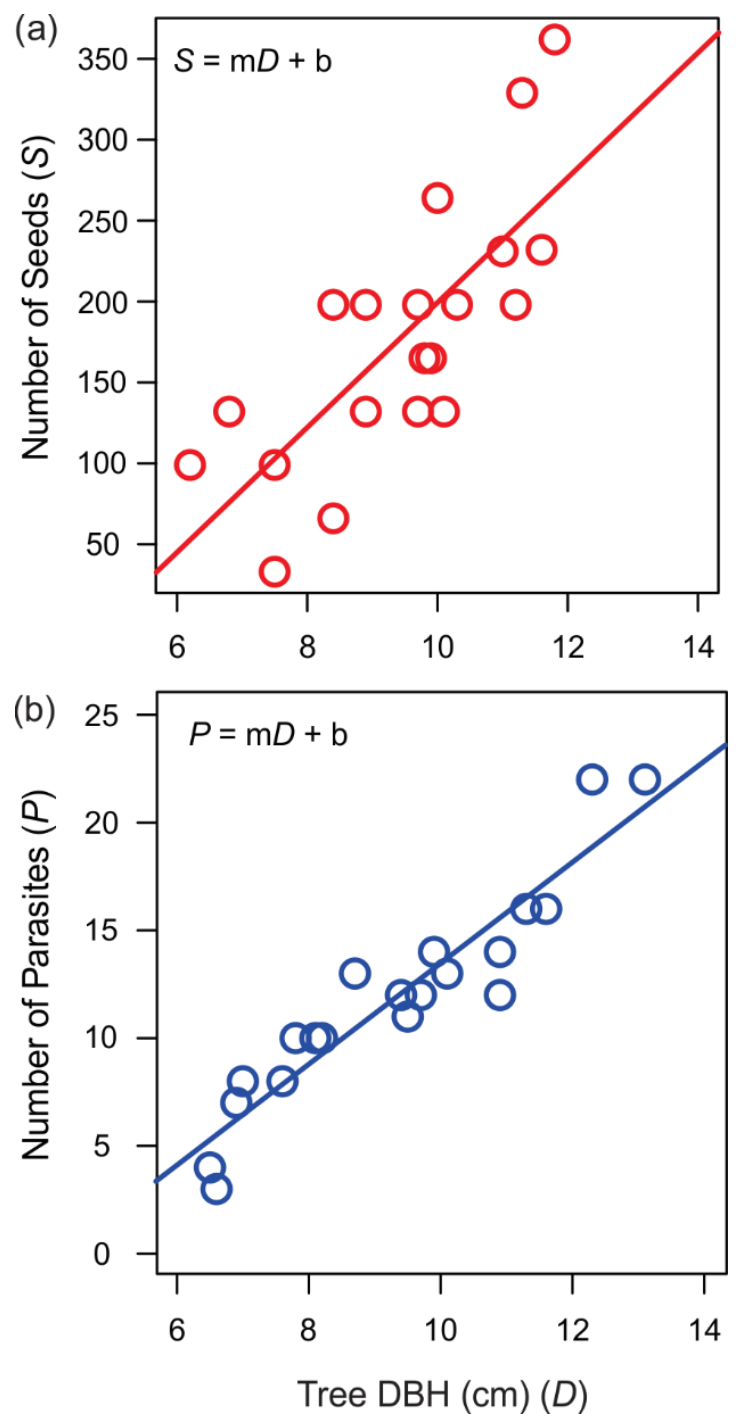

Figure 5.3.2. The relationship between (a) the number of seeds (S) and tree DBH (D) for unparasitised female trees, and (b) the number of parasites $(P)$ and tree DBH (D) for male trees (no seeds). The equation in each figure shows the slope $(\mathrm{m})$ and intercept (b) for each relationship used to solve for $D$.

To test if the difference in the number of parasites between male and female trees was a result of parasite-offspring competition for female resources, I ran the full model using a second empirical data set collected from a different subset of randomly selected hosts following the methods described above. Specifically, I tested whether $P_{\sigma^{\prime}}=P_{q}+\alpha_{P S} S$ by performing a linear regression with $P_{q}+\alpha_{P S} S$ as the predictor variable and $P_{\delta}$ as the response variable. I extracted the upper and 
lower $95 \%$ confidence intervals $(\mathrm{Cl})$ for the slope of the regression. If the line of isometry, a 1:1 relationship where $P_{\delta}=P_{T}+\propto_{P S} S$ exactly, falls between the confidence intervals of the linear regression, then the model verifies the hypothesis that male biased susceptibility is a result of parasite-offspring competition for female resources.

\subsubsection{Testing the alternative explanation for MBS}

One of the most common explanations for the pattern of MBS to parasites is the androgen based immune response whereby testosterone in males increases male sexual traits but simultaneously reduces the immune response to pathogens and parasites (Zuk \& McKean 1996). Conversely, plants do not have an analogous system to the androgen based immune response in animals. However, plants do have physiological defences which help prevent attacks of parasites, herbivores and disease, such as chemical responses to damage of foliage, or physical structures such as toughness of leaves (Ågren et al. 1999; Cornelissen \& Stiling 2005). In Chapter 3, I found bark thickness was the only significant tree defence against larvae attack on interspecific hosts (Yule \& Burns, in review). To test whether tree defences differed between male and female hosts, I determined the bark thickness of 20 male and 20 female $A$. serrata trees by collecting a $10 \mathrm{~mm} \times 20 \mathrm{~mm}$ section of bark from both north- and south-facing aspects of each tree. Bark thickness was measured using digital callipers to assess the bark thickness from two opposite ends of each bark piece. Means \pm se were calculated for both male and female trees. A Welch's two-sample t-test was used to assess the differences in bark thickness (immunity) between male and female trees after controlling for tree size.

All statistical analyses were carried out in R ( $R$ Core Team 2015). 


\subsection{Results}

\subsubsection{Meta-analysis}

In total, 188 studies met my criteria consisting of $>149,000$ individual host organisms inspected for parasite prevalence or intensity. From these studies, I compiled the most comprehensive host-parasite database in existence, comprising 461 host species-parasite pairings; 409 host-parasite pairings from 70 animal families (Appendix Table A3) and 52 host-parasite pairings from 22 plant families (Appendix Table A2). From the animal host-parasite pairings, 293 out of 409 had MBS, which was significantly higher than would be expected if bias was random (Bernoulli: $P<0.001$ ). Similarly, 44 out of 52 plant host-parasite pairings had MBS, which again was significantly higher than would be expected by chance (Bernoulli: $P$ $<0.001$ ). However, owing to the overrepresentation of many host organisms belonging to the same higher taxonomic groups, these tests are likely overpowered.

To account for the phylogenetic relatedness of the host organisms in the data sets, I grouped the organisms into taxonomic families and ran computer simulations to provide an expected frequency distribution of MBS for each family group. In support of the Bernoulli trials, I found that MBS to parasites at the family level was significantly different than if susceptibility was occurring at random. The differences between the observed and expected frequency of male-bias parasitism in family groups was significant for animals $(W=3823, P<0.001$, Fig 5.4.1a) and plants $(\mathrm{W}=$ 396, $P<0.001$, Fig 5.4.1b). To test if the pattern of MBS was different between animals and plants, I assessed the observed frequency of MBS between the animal and plant family groups and found no significant difference in frequencies of MBS ( $W$ $=712.5, P>0.05$, Fig 5.4.1). The pattern of MBS is therefore not occurring at random and is the same across animals and plants. 

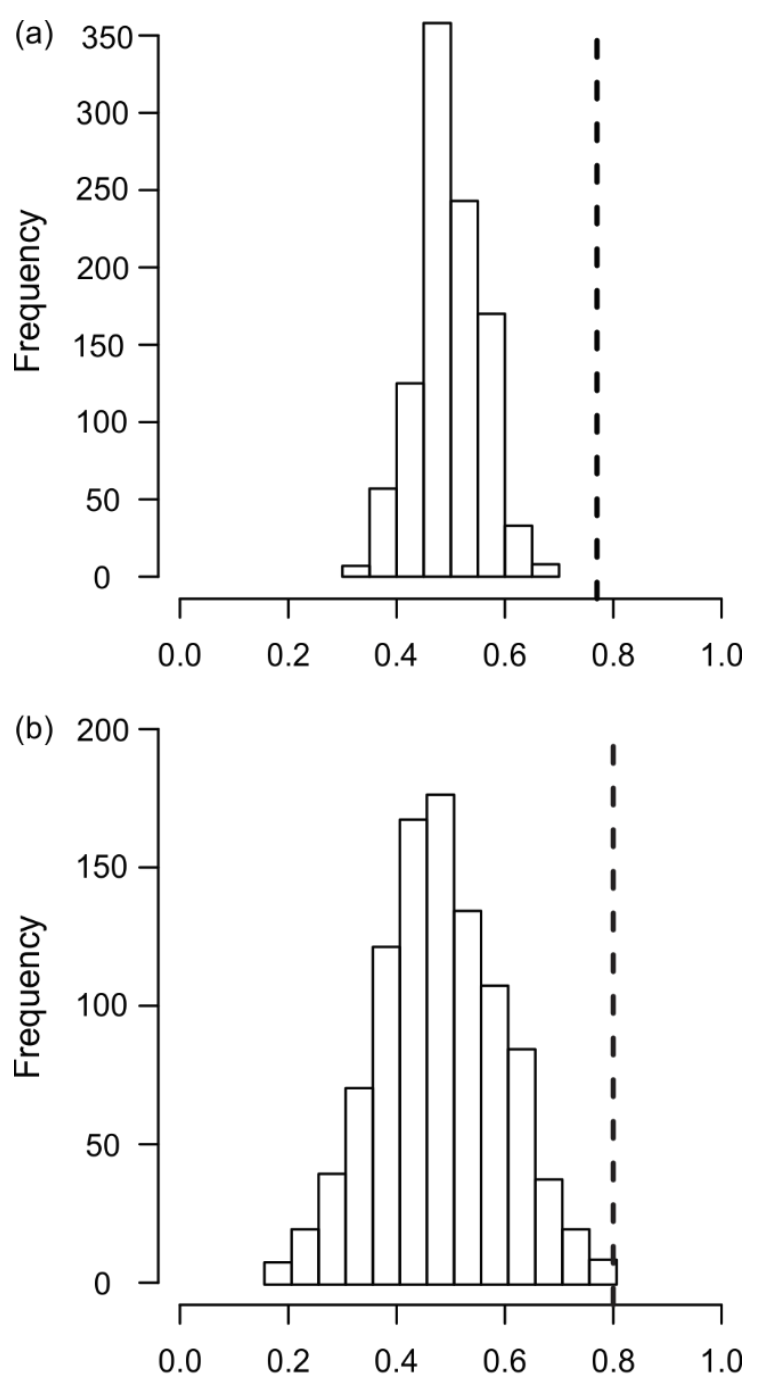

Expected bias in Families

Figure 5.4.1. Frequency of expected bias from 1000 random simulations for: (a) animal families $(n=70)$ and (b) plant families $(n=22)$. " 0 " indicates an entirely female bias and " 1 " indicates an entirely male bias. Dashed lines indicate the mean observed bias.

\subsubsection{Empirically testing the theoretical model}

To test the hypothesis that the difference in the number of parasites between male and female trees was a result of parasite-offspring competition for female resources, I developed a theoretical model whereby I predicted that $P_{i}=P_{q}+\propto_{P S} S$. I ran a linear regression with $P_{+}+\propto_{P S} S$ and the predictor variable and $P_{\delta}$ as the 
response. Results indicate there was a significant relationship between $P_{q}+\propto_{P S} S$ and $P_{\delta^{\prime}}(\mathrm{r} 2=0.92, \mathrm{t}=14.57, \mathrm{df}=17, \mathrm{P}<0.001$, Figure 5.4.2.). The slope of the relationship did not significantly differ from isometry at the $95 \%$ confidence interval (slope: 1.06, lower $\mathrm{Cl}: 0.90$, upper $\mathrm{Cl}: 1.21$ ) indicating the theoretical model accurately describes the empirical data. Furthermore, these results support the hypothesis that the process driving male-biased susceptibility is that parasites and offspring compete for female resources.

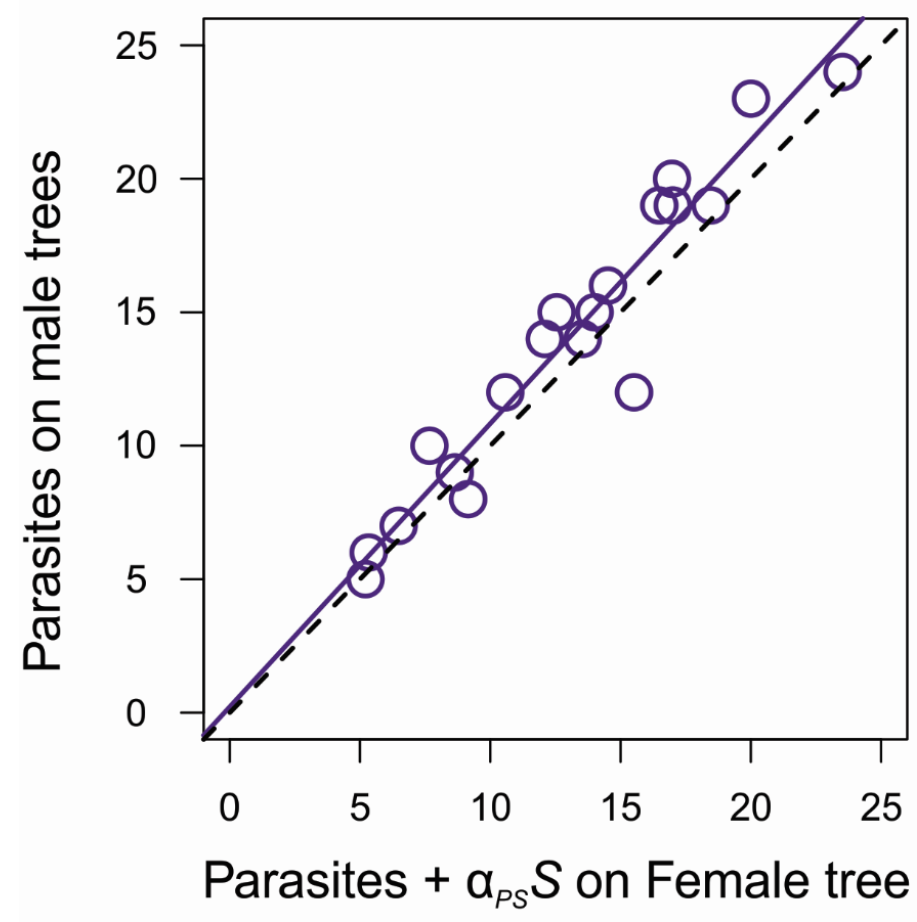

Figure 5.4.2. Relationship between the number of parasites on female trees $\left(P_{\text {}}\right)$ plus the coefficient equating the energetic cost of seeds to the energetic cost of parasites $\left(\propto_{N S} S\right.$, ) and the number of parasites on male trees ( $\left.\mathrm{P}_{3}\right)$ (Slope: 1.06, lower $\mathrm{Cl}=0.90$, upper $\mathrm{Cl}=1.21$ ). Solid line is line of best fit from linear regression; dashed line is the line of isometry $(1: 1)$. 


\subsubsection{Immune-defence hypothesis}

To test whether the primary defensive trait in host trees differed between host sexes, making one sex more susceptible to attack than the other, I assessed bark thickness for male and female host trees. No significant difference was found in bark thickness between male and female host trees $(\mathrm{t}=0.47913 \mathrm{df}=18.961, P=0.63$, Figure 5.4.3), indicating that neither host was more susceptible than the other to parasite attack in terms of primary defences. These results refute the immunedefence hypothesis as an over-arching explanation for MBS across animals and plants.

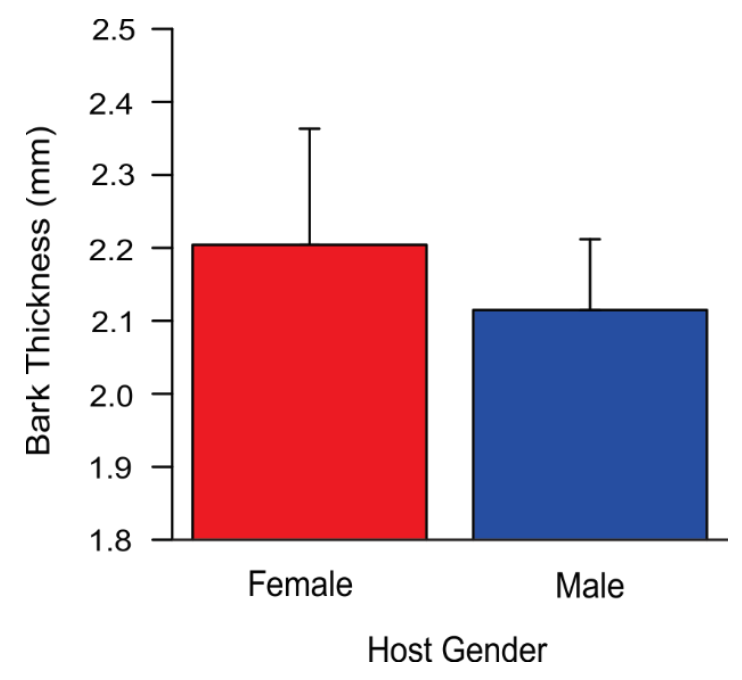

Figure 5.4.3. Difference between bark thickness on female (red) and male (blue) Aristotelia serrata host trees.

\subsection{Discussion}

Male-biased susceptibility (MBS) is prolific across multiple taxonomic groups of animals. Similarly, MBS also occurs in plants. Here, I presented the first theoretical model that explains MBS in the A. serrata - A. virescens host-parasite system, which demonstrates that parasites are in direct competition with offspring for 
female resources. However, I found no evidence for differential resistance, i.e. plant "immunity" between host plant sexes.

To my knowledge, the database of host-parasite pairings compiled in this research is the most comprehensive in the literature to date. Although MBS has been the focus of much debate, the meta-analysis comprising 188 studies supports a highly significant pattern of MBS for both animals and plants. I found a significant difference in the mean number of susceptible male hosts within family taxonomic groups compared with what would be expected by chance in both animals and plants. Traditionally, MBS in animals has been explained as a result of a reduced immune response in males and/or an increased cost of reproduction in females. This meta-analysis revealed 38 animal studies that specified the androgen-immune system as a broad-scale explanation for MBS (see Appendix Table A.1. for references). However, organisms that lack testosterone or analogous systems, such as arthropods and plants, still show a significant MBS. A review of immune system responses in vertebrates by Klein (2004) concluded the androgen-immune system was not an overarching explanation for MBS in vertebrates. Similarly, Zuk and McKean (1996) and Sheridan et al. (2000) also contested the androgen-immune theory as the underlying process for patterns in MBS, primarily owing to susceptibility in organisms who lack the androgen-immune system.

For animals, multiple studies suggest morphological, ecological and behavioural factors underpinning the differences in parasite loads amongst host sexes (Table 1). For example, sexual size dimorphism (SSD), where the sexes differ significantly in body size or morphology as a result of sexual selection, has been proposed as the driving force behind MBS (Schall \& Marghoob 1995; Isomursu et al. 2006; Harrison et al. 2010); however, other studies oppose this (Morand et al. 2004; Patterson et al. 2008). Similarly, foraging activities, dispersal and home-range sizes (Moravvej et al. 2016), dietary differences (Reimchen \& Nosil 2001; Davies et al. 2008; Grzybek et al. 2014), reproductive behaviours (Soliman et al. 2001; Imasuen et al. 2011), seasonal factors (Halvorsen et al. 1985; Schall et al. 2000a; Krasnov et al. 2005a) and environmental conditions (Schall et al. 2000a) have all been proposed 
as the underlying mechanisms driving the differences in susceptibility between sexes.

Plants have a similar suite of plausible explanations that potentially underpin MBS. Quality of nutritional resources (Hjältén 1992; Boecklen 1993; Verdú et al. 2004; Uribe-Mú \& Quesada 2006a; Lucero García-García \& Cano-Santana 2015), defences such as leaf toughness and defensive compounds (Jing \& Coley 1990; Verges et al. 2008; Tsuji \& Sota 2010), reproduction (Feller et al. 2002; CepedaCornejo \& Dirzo 2010), phenology (Boecklen et al. 1990) and distance to unpalatable plants (Graff et al. 2013) have all been proposed as an explanation for sex-based bias in susceptibility to parasites. Primarily, plants lack an androgen-immune system and therefore oppose the immune-defence hypothesis as an overarching explanation for MBS in animals and plants. However, much work has been done on plant defences against parasites (reviewed by Cornelissen and Stiling 2005). In Chapter 3 , bark thickness was established as the primary tree defence preventing attack by $A$. virescens larvae; tree species with thick bark had fewer parasites whilst tree species with thin bark had more parasites (Yule and Burns, Unpubl.). I investigated the difference in bark thickness between male and female trees as the primary defence against attack. I found no significant difference in bark thickness between male and female host trees, ruling out tree defences for the pattern of MBS observed in the studied system. Defences are likely an important aspect of inter-specific host choice owing to the co-evolutionary arms race between parasites and hosts, but defences are perhaps not a strong selection pressure for intra-specific host use.

An advantage of investigating MBS in plant hosts is that plants are sessile and lack the complex behaviours of animals, thus removing many confounding factors that may obscure true patterns in sex-biased susceptibility to parasites. Potentially, if animals could be investigated independent of the behaviours suggested previously as causes of MBS, many of these suggestions could potentially be discounted. As with all theories or rules, exceptions will exist. Perhaps the occurrences of female bias in some taxonomic families results from the different reproductive regime of the female hosts, their various lifestyle nuances, or the life 
history of the parasite. Additionally, organisms don't exist in isolation and are subject to selection pressures from multiple antagonists at any given time. It is possible that numerous selection pressures are sufficiently strong that they supersede the strength of competition for resources with offspring. Further research on the fitness of parasites that infect either male or female hosts would provide significant insight into the strength of selection pressures parasites face when selecting between host sexes. Furthermore, not all resources may be equal. Hosts that display non-MBS are potentially a result of the specific resources utilised by the parasites. The dioecious tree-parasitic larvae system used to test the theoretical model in this study offered a unique opportunity to investigate a parasite who is directly feeding on the resources utilised by offspring (phloem); however, this may not be the case in all host-parasite pairings.

Regardless, the most parsimonious explanation for the over-arching MBS phenomenon is that female hosts are already depleted of resources through competition with their offspring. All host organisms parallel islands representing discrete, finite pools of resources (Kuris et al. 1980). In species where females retain and nourish offspring, from the moment of syngamy, offspring are parasites sensu lato, depending entirely on female resources for survival. The competitive nature between mothers and offspring deplete the pool of resources potentially available to parasites. This burden of offspring on female hosts has no parallel in male hosts post-syngamy for species where males invest no resources in offspring development. What female resources are used by offspring, are resources not available for parasites. Therefore, the already depleted pool of resources in females is likely to have significant implications for the number of parasites sensu stricto that can be supported by a host at any given time. The theoretical model predicts the number of parasites in female hosts plus the number of parasites equivalent to their offspring burden (calculated by the competition coefficient) is equal to the total parasite load in male hosts. My empirical data support this hypothesis. I tested the proof of the theoretical model by giving the burden of offspring to male hosts, which resulted in males having a significantly similar parasite load to females. Interestingly, several studies indicated the pattern of MBS does not hold up in juveniles (Butcher et al. 2002; Diaz and Alonso 2003; Isomursu et al. 2006; Dare and Forbes 2009; 
Krichbaum et al. 2009). Potentially, the lack of difference in parasite intensity or prevalence in juveniles may be a result of juveniles not yet reaching sexual maturity. Juvenile females have no offspring burden to bear making the pool of resources the same for males and females. However, this remains unresolved.

The implications for this model are significant. For hosts, being susceptible to parasites has been implicated in altering social hierarchies, host decision making, mating behaviours, sexual reproduction, energetic costs owing to immune response or defences against parasites and reduced fecundity (Hurd 2001; Morales-montor et al. 2004). Understanding what makes a host susceptible also provides vital information when investigating virulence and transmission of parasites, and the likelihood of parasites colonising new locations. My model provides the first explanation for MBS in our study system and may be applicable to other hostparasite systems where females retain and nourish the offspring post syngamy. 


\section{Chapter 6. General Discussion}




\subsection{Thesis Summary}

The results of this thesis present the first investigation of New Zealand's largest endemic moth Aenetus virescens, the relationship with their host trees, and the interaction with their avian predator. I used a system-based approach to investigate complex parasite-host evolutionary ecology using $A$. virescens (hereafter "larvae") as a model system. First, the results of Chapter 2 showed larvae followed the common pattern of parasite aggregation whereby many hosts had few parasites and few hosts had many (Chapter 2). The mechanism driving intraspecific parasite aggregation was host size rather than resource availability or density-dependent competition. Second, the results of Chapter 3 suggest larvae host specificity at multiple locations is a direct result of bark thickness, a tree defence, rather than nutritional quality or abundance of hosts. Third, the results of Chapter 4 indicate cryptic webbing did not protect larvae from attack; instead, cryptic webbing aids parasite thermoregulation, suggesting that crypsis is non-adaptive. Finally, the results of Chapter 5 indicate a significant occurrence of male-biased susceptibility (MBS) in both animals and plants and that MBS was a direct result of parasites competing with offspring for female resources.

Parasites are ubiquitous and represent the most common life strategy on Earth (Lafferty et al. 2006; Dobson et al. 2008). All parasites require hosts for nutrition, shelter and, ultimately, survival (Tscharntke 1992; Lafferty et al., 2008; Johnson et al., 2010; Goedknegt et al., 2012; Friman \& Buckling 2013) and the antagonistic relationships between parasites and their hosts determine population and community structure in natural ecosystems (Pimm 1979; Pimm 1980a; Paine 1980; Pimm et al., 1991; Polis et al., 1997; Thompson et al., 2007; Lafferty et al., 2008; Chase 2013). Yet the complex interactions between parasites and their hosts often limit generalisations that can be made regarding specific host or parasite species (Poulin \& Forbes 2011). Parasites present a possibly greater challenge than free-living species for estimating abundance, diversity or the implications of their associations for ecology and evolution (Dobson et al. 2008; Gómez \& Nichols 2013). Thus, our understanding of complex parasite-host relationships remains lacking. 
Overall, the results of this thesis support existing theories of parasite aggregation and host specificity from a novel perspective. Furthermore, results support a newly emerging paradigm shift in animal camouflage evolution, and propose a unified explanation for male-biased susceptibility in animals and plants. These exciting results help to bridge the gap in our knowledge of the processes underpinning key patterns of host-parasite ecology and evolution, combining and expanding interdisciplinary research interests with potentially significant advances in our understanding of complex antagonistic interactions.

\subsection{Synthesis}

\subsubsection{Intraspecific parasite aggregation on hosts}

Parasites are ubiquitous and their interactions with hosts shape populations and ecosystems (Poulin 2007). The right-skewed distribution of parasites among hosts is a fundamental pattern of host-parasite interactions; many hosts have few parasites and few hosts have many (Shaw et al. 1998; Tschirren et al. 2007; Calabrese et al. 2011; Poulin \& Forbes 2011; Poulin 2013). Concurrently, my results are consistent with these findings (Chapter 2) as larvae followed the same aggregated distribution among individual host trees. A prominent pattern in parasite ecology, the mean number of parasites per individual host commonly scales with host size (see (Poulin 2000, 2005; Poulin \& Morand 2000), 2005; Poulin and Morand, 2000). For example, a meta-analysis of 76 different fish host-parasite relationships found positive correlations between host size and parasite intensity, although relationship significance varied (Poulin 2000). Concurrently, my results indicate host size was a significant predictor of parasite infrapopulation size.

Conspecific competition occurs when individuals from the same species are vying for the same resources. Larvae often have multiple individuals in one host tree and all live $\sim 6$ years feeding on host tree phloem. However, larvae growth rate was not influenced by host size or the size of parasite infrapopulation suggesting 
aggregation is not density-dependent. Similarly, Duan et al. (2013) found no densitydependent competition in Agrilus planipennis (Coleoptera: Buprestidae), who solely feed on phloem. One explanation is that wounds from feeding insects act as sinks, directing plant resources to these areas in response to feeding (Kehr 2006; Muilenburg et al. 2013). In fish host-parasite relationships, low parasite intensities do not constrain parasite growth, whereas large parasite intensities produce resource competition and intensity-dependent growth (Poulin and Morand, 2000; Poulin, 2005; Saldanha et al., 2009). Potentially, larvae may occur in sufficiently small numbers, or alternatively regulate recruitment, ensuring resources are not limited by increasing parasite numbers.

Overall, the results from Chapter 2 indicate larvae are aggregating on larger hosts, yet do not receive additional rewards. I found no evidence for densitydependent effects of parasite infrapopulations; therefore, host size appears to be the most parsimonious explanation for the pattern of parasite aggregation on hosts.

\subsubsection{Host specificity}

At the community level, parasite aggregation also occurs inter-specifically. Results from Chapter 3 indicate that larvae are generalist parasites, aggregating on several host species. Furthermore, larvae use the same suite of hosts at multiple locations regardless of host abundance and differing forest composition. Parasite adaptations to their local hosts is a fundamental evolutionary process that determines host-parasite specialisation (Gotthard et al. 2004). Parasites benefit directly from host nutritional quality; however, host-parasite relationships also arise from co-evolutionary arms races between host defences and a parasites' ability to circumvent these (Dawkins \& Krebs 1979; Langmore et al. 2003). Similar to Chapter 2, rewards from host tree did not explain larvae host specificity, indicating that parasites are not utilising hosts which offer the greatest rewards (Chapter 3 ). However, generalist parasites must invest in an array of potentially costly counteradaptations to overcome defences of several hosts (Poulin 2007; Sears et al. 2012). Whilst wood density did not explain larvae host specificity, bark thickness was 
significantly related to a larvae's ability to use a host. External defences of hosts, such as bark thickness, appear to be the primary mechanism driving host specificity for larvae, likely due to increased energy requirements needed to overcome the external barrier.

A parasite's contemporary ecological niche determines the likelihood of parasite extinction if hosts become scarce, and the likelihood of the parasite becoming established in new areas (Poulin et al. 2006; Poulin and Keeney 2008). Host specificity is therefore particularly important for larvae whose long arboreal life stage and restricted distribution expose them to even small perturbations in host populations. Owing to significant difficulties in quantifying parasite abundance and diversity, the conservation status of most parasite species is unknown; however, it is possible many are endangered or threatened as a result of significant losses in their host species (Gómez \& Nichols 2013). Moreover, host ranges are likely to change significantly under anticipated climatic conditions (Davis \& Shaw 2001). Parasites must keep pace with their host's range shifts, particularly if intermediate life stages require multiple host species that may fair differently under climatic pressures. Conversely, parasites are often severely detrimental to their hosts, causing morbidity and mortality. The likelihood of a new pest, pathogen or disease emerging in a new location is entirely reliant on the presence of a host in which the parasite can successfully infect (Lootvoet et al. 2013). Several emerging disease in humans, for example, were historically well established amongst primates; generalist parasites are notorious for altering their host specificity, colonising previously unsusceptible hosts (Pedersen et al. 2005).

Our understanding of the intimate associations of parasites and their hosts, both intra- and inter-specifically, remains unresolved, lacking overarching generalisations (Poulin \& Forbes 2011). To fully appreciate the cost of losing such associations, the ecological impact parasites have for ecosystems as a whole must be considered. 


\subsubsection{Adaptive consequences of camouflage}

Predation is one of the most significant selection pressures an organism will face. For parasites, predation normally occurs on their free-living life stages, or accidentally through predation of their host (Goedknegt et al. 2012). Anti-predator adaptations - in particular, camouflage — have been considered a seminal example of natural selection since Darwin (1858) and Wallace (1870). Whether camouflage is an anti-predator adaptation or a non-adaptive consequence of selection acting on another primary function of colour (e.g. communication or thermoregulation) (Nylin et al. 2001; Stuart-Fox \& Moussalli 2009) is an unresolved interdisciplinary problem (Stevens \& Merilaita 2009; Troscianko et al. 2013; Stevens \& Tevens 2015) with quantitative studies only emerging in the last 15 years (Stevens \& Tevens 2015). I directly assessed the adaptive advantages of camouflage, testing larvae survivability in a long term field experiment (Chapter 4). Results indicate webbing was cryptic to predating kaka when viewed in avian tetrahedral colour space. However cryptic webbing did not protect larvae from attack.

Insects are ectothermic, and temperature plays a significant role in growth, development, and metabolic processes (Ruf \& Fiedler 2002; Kührt et al. 2005). I found temperatures were significantly warmer inside larval tunnels with webbing, compared to larval tunnels without webbing (Chapter 4). Furthermore, temperatures were highest around the feeding scar where larvae feed nocturnally. The implications for this are two-fold: the hotter area may remain warmer for longer when larvae are active and utilise the area to feed, potentially aiding metabolic processes and activity patterns (Ruf \& Fiedler 2002). Additionally, warmer temperatures may encourage the tree healing processes, specifically the increase of cambium regeneration, increasing flow of phloem sap to larvae (Kehr 2006; Deslauriers et al. 2008). Further work on larval body temperature and the internal thermoregulation of tunnels is required to fully elucidate the advantages of increased temperature resulting from webbing structures. 
Whilst webbing does not increase larvae survivability via crypsis, the presence of webbing still offers a degree of concealment compared to tunnels without webbing (Chapter 4). Primarily, larvae would be fully exposed to predators and abiotic factors whilst feeding if the webbing was not present. Moreover, the webbing may provide a barrier to organisms who utilise larvae tunnels. For example, tree weta Hemideina sp. (Orthoptera: Anostostomatidae) use the tunnels as refuges once larva emerge (Green 2005). As the webbing is made from larval silk, frass and masticated wood pieces, it may also provide chemical signalling to conspecifics regarding territory; feeding scars never overlap, but adjoin each other when space is limited.

\subsubsection{Male-biased host susceptibility}

Male-biased susceptibility (MBS) is prominent across multiple taxonomic groups of animals and has recently been observed in plants. In the past 20 years, several reviews have addressed MBS in specific taxonomic groups, i.e. small mammals (Krasnov et al. 2012), arthropods (Sheridan et al. 2000), vertebrates (Klein 2004), birds (McCurdy et al. 1998) and plants (Ågren et al. 1999). However, plant and animal groups have not yet been amalgamated, despite evidence supporting similar magnitudes of MBS in both animals and plants. Here, I present a model that explains MBS in plants and animals whereby parasites are in direct competition with offspring for female resources in species where females retain and nourish offspring post-syngamy (Chapter 5). I tested the model empirically, giving male hosts the burden of offspring and removing the burden of offspring from females.

The implications for my empirically driven model to explain MBS are substantial. First, the significance of offspring as parasites has broad-scale implications for organisms where females retain and nourish offspring post-syngamy, directly influencing their susceptibility to pathogens and parasites. For males who are more susceptible to parasites, alterations to social hierarchies, host decision making, mating behaviours, sexual reproduction, energetic costs due to immune response or defences against parasites are all possible outcomes of increased parasite burden 
(Hurd 2001; Morales-montor et al. 2004). As susceptibility varies between sexes of the same species, males and females are likely to encounter different selection pressures as a result of differential degrees of parasitisation. In particular, the degree of dioecy in plants (Ashman 2002) and the sex ratio (Cornelissen \& Stiling 2005b) or SSD in animals and plants (Kavanagh et al. 2011) could be significantly altered by increased parasite loads in males. Similar to understanding the mechanisms driving host specificity (Chapter 2), the underlying cause of hosts susceptibility provides vital information when assessing parasite virulence, transmission and the likelihood of parasites colonising new locations (Sears et al. 2012).

The most parsimonious explanation for the over-arching MBS phenomenon is that female hosts are already depleted of resources through competition with their offspring. All host organisms parallel islands representing discrete, finite pools of resources (Kuris et al. 1980). For female organisms that retain and nourish offspring post syngamy, offspring are parasites sensu lato, depending entirely on female resources for survival. The competitive nature between mothers and offspring deplete the pool of resources potentially available to parasites. This burden of offspring on female hosts has no parallel in male hosts post syngamy. The female resources utilised by offspring are not available for parasites. Therefore, the already depleted pool of resources in females is likely to have significant implications for the number of parasites sensu stricto that can be supported at any given time. The model I developed in Chapter 5 presents the most parsimonious explanation for MBS to parasites and the first explanation for MBS in the $A$. serrata $-A$. virescens host-parasite system. The model may also be applicable to other host-parasite systems where females retain and nourish offspring post-syngamy.

\subsection{Conclusions and Future Directions}

To my knowledge, this thesis presents the first investigations of the natural history of the Aenetus virescens larvae, their relationships with host trees and the interactions with their avian predator. As a result, much of the work described here are the first steps to further explorations of this remarkable system and the complex 
interactions therein. Whilst the study species themselves present a degree of novelty, this does not detract from the broad-scale contributions that this research makes to parasite evolutionary ecology.

Larvae show intraspecific aggregation on hosts and interspecific preference for one or two host species regardless of abundance (Chapters 2 and 3). I recommend further investigation into: i) the stimuli cueing larvae towards host trees; and ii) whether parasite aggregation and host specificity follow the patterns presented here across parasite and host geographic ranges. Based on the results of Chapters 1 and 2, ongoing research into whether larvae are ecosystem engineers in regenerating New Zealand forest is currently underway.

The broad-scale approach of Chapter 4 supports the exciting, newly emerging paradigm shift that suggests camouflage in animals has potentially evolved in response to abiotic factors rather than biotic signalling. My results challenge traditional theories of camouflage and I present evidence that crypsis is nonadaptive, rather a by-product thermoregulation. Primarily, the assumption of camouflage as an anti-predator adaptation only holds true if the survivability of prey is increased as a result of camouflage. However, few studies have specifically tested survivability of prey in relation to crypsis, and fewer have done so whilst accounting for the visual system of the predator. It is crucial that future research address how crypsis, or alternative modes of camouflage, alter survivability compared with noncamouflaged prey. Only then can reliable conclusions about the adaptive consequences of camouflage be reached. For my study system, an interesting question arose regarding how the visually orientated kaka locate cryptic larvae. Despite webbing being visually cryptic to kaka, attacks are precisely on larvae tunnels. During field work I observed kaka rapidly drumming their tongues on the bark of trees. I am currently investigating the possibility that kaka are using selfgenerated acoustic cues to identify presence, position and size of larvae concealed inside a host tree. 
Perhaps the most exciting outcome of this thesis is the development of an explanation for MBS in plants and animals. Traditionally, research has focused heavily on this pattern in animals, with testosterone suppressing the immune system of males as the principal explanation for MBS; only recently have plants been considered. I compiled the largest database of host-parasite pairings and showed that MBS is significant in plants and animals. The model I developed in Chapter 5 presents the most parsimonious explanation for MBS to parasites whereby parasites compete with offspring for female resources. As an extension of this research, I am currently investigating the difference in parasite virulence between male and female hosts.

Overall, this thesis presents a significant contribution to parasite evolutionary ecology and their role in shaping ecosystems. The results herein help further our understanding of complex antagonistic relationships between parasites and their hosts, presenting novel theories on which future research can be built.

\section{References}


Abell KJ, Duan JJ, Bauer L, et al. (2012) The effect of bark thickness on host partitioning between Tetrastichus planipennisi (Hymenoptera: Eulophidae) and Atanycolus spp. (Hymenoptera: Braconidae), two parasitoids of emerald ash borer (Coleop: Buprestidae). Biol Control 63:320-325. doi: 10.1016/j.biocontrol.2012.08.009

Acuna-Soto R, Maguire JH, Wirth DF (2000) Gender distribution in asymptomatic and invasive amebiasis. Am J Gastroenterol 95:1277-1283. doi: 10.1016/S0002-9270(99)00584-5

Agren J (1987) Intersexual differences in phenology and damage by herbivores and pathogens in dioecious Rubus chamaemorus L. Oecologia 72: 161-169

Ågren J, Danell K, Elmqvist T, et al. (1999) Sexual dimorphism and biotic interactions. Gend Sex Dimorphism Flower Plants 217-246. doi: 10.1007/9783-662-03908-3_8

Aguilar-Delfin I, Homer MJ, Wettstein PJ, Persing DH (2001) Innate resistance to Babesia infection is influenced by genetic background and gender. Infect Immun 69:7955-7958. doi: 10.1128/IAI.69.12.7955-7958.2001

Ahman I (1997) Growth, herbivory and disease in relation to gender in Salix viminalis L. Oecologia 111:61-68. doi: 10.1007/s004420050208

Alexander J, Stimson W (1988) Sex-hormones and the course of parasitic infection. Parasitol Today 4:189-193

Alliende AMC (1989) Demographic studies of a dioecious tree . II. The distribution of leaf predation within and between trees. J Ecol 77:1048-1058

Amman G (1969) Mountain pine beetle emergence in relation to depth of lodgepole pine bark. Ogden, Utah 84401

Anton V, Hartley S, Wittmer H (2015) Survival and growth of planted seedlings of three native tree species in urban forest restoration in Wellington, New Zealand. N Z J Ecol 39:170-178

Apanius V, Yorinks N, Bermingham E, Ricklefs R (2000) Island and taxon effects in parasitism and resistance of Lesser Antillean birds. Ecology 81:1959 - 1969. 
Arnold KE, Adam A, Orr KJ, et al. (2003) Sex-specific survival and parasitism in three-spined sticklebacks: seasonal patterns revealed by molecular analysis. J Fish Biol 63:1046-1050. doi: 10.1046/j.1095-8649.2003.00195.x

Ashman TL (2002) The role of herbivores in the evolution of separate sexes from hermaphroditism. Ecology 83:1175 - 1184

Augustyn WJ, Anderson B, Stiller M, Ellis AG (2013) Specialised host-use and phenophase tracking in restio leafhoppers (Cicadellidae: Cephalelini) in the Cape Floristic Region. J Insect Conserv 17:1267-1274. doi: 10.1007/s10841013-9608-2

Baboolal S, Rawlins SC (2002) Seroprevalence of toxocariasis in schoolchildren in Trinidad. Trans R Soc Trop Med Hyg 96:139-143. doi: 10.1016/S00359203\%2802\%2990281-6

Bagamian KH, Heins DC, Baker JA (2004) Body condition and reproductive capacity of three-spined stickleback infected with the cestode Schistocephalus solidus. J Fish Biol 64:1568-1576. doi: 10.1111/j.1095-8649.2004.00411.x

Bañuelos M-J, Kollmann J (2011) Effects of host-plant population size and plant sex on a specialist leaf-miner. Acta Oecologica 37:58-64. doi: 10.1016/j.actao.2010.11.007

Banuelos MJ, Obeso JR (2003) Maternal provisioning, sibling rivalry and seed mass variability in the dioecious shrub Rhamnus alpinus. Evol Ecol 17:19-31. doi: 10.1023/A

Barber I (2005) Parasites grow larger in faster growing fish hosts. Int J Parasitol 35:137-43. doi: 10.1016/j.jpara.2004.11.010

Bates D, Maechler M, Bolker B, Walker S (2014) Ime4: Linear mixed-effects models using Eigen and S4. J Stat Software 67:1-48. doi: 10.18637/jss.v067.i01

Begum S, Nakaba S, Bayramzadeh V, et al. (2008) Temperature responses of cambial reactivation and xylem differentiation in hybrid poplar (Populus sieboldii x P. grandidentata) under natural conditions. Tree Physiol 28:1813-1819. doi: 10.1093/treephys/28.12.1813 
Behnke JM, Lewis JW, Zain SN, Gilbert FS (1999) Helminth infections in Apodemus sylvaticus in southern England: interactive effects of host age, sex and year on the prevalence and abundance of infections. J Helminthol 73:31-44. doi: $10.1017 / \mathrm{S} 0022149 \times 99000049$

Belan I, Bull C (1991) Host detection by four Australian tick species. J Parasitol 77:337-340

Bertolino S, Wauters LA, Bruyn L De, et al. (2003) Prevalence of Coccidia parasites (Protozoa) in red squirrels (Sciurus vulgaris): effects of host phenotype and environmental factors. Oecologia 137:286-295

Blackledge T (1998) Signal conflict in spider webs driven by predators and prey. Proc Biol Sci 265:1991-1996

Blanco G, Seoane J, de Puente J, et al. (1999) Showiness, non-parasitic symbionts, and nutritional condition in a passerine bird. Ann Zool Fennici 36:83-91

Blasco-Costa I, Poulin R (2013) Host traits explain the genetic structure of parasites: a meta-analysis. Parasitology 140:1316-22. doi: 10.1017/S0031182013000784

Blick R, Bartholomew R, Burrell T, Burns KC (2008) Successional dynamics after pest eradication in the Karori Wildlife Sanctuary. New Zeal Nat Sci 33:3-14

Boecklen WJ, et al. (1993) Sex-biased herbivory in Ephedra trifurca:the importance of sex-by-enviroment interactions. Ecol 96:49-55

Boecklen WJ, Price PW, Mopper S (1990) Sex and drugs and herbivores: sexbiased herbivory in arroyo willow (Salix lasiolepis). Ecology 71:581-588. doi: $10.2307 / 1940311$

Bond AB (2007) The evolution of color polymorphism: crypticity, searching images, and apostatic selection. Annu Rev Ecol Evol Syst 38:489-514. doi: 10.1146/annurev.ecolsys.38.091206.095728

Bond BJ (2000) Age-related changes in photosynthesis of woody plants. Trends Plant Sci 5:349-53

Brown DS, Symondson WOC (2014) Sex and age-biased nematode prevalence in reptiles. Mol Ecol 23:3890-3899. doi: 10.1111/mec.12688 
Bundy D (1988) Sexual effects on parasite infections. Parasitol Today 4:186

Burkett-Cadena ND, Bingham AM, Unnasch TR (2014) Sex-biased avian host use by arbovirus vectors. R Soc open Sci 1:140262. doi: 10.1098/rsos.140262

Burns B, Innes J, Day T (2012) The use and potential of pest-proof fencing for ecosystem restoration and fauna conservation in New Zealand. In: Somers MJ, Hayward M (eds) Fencing for conservation: restriction of evolutionary potential or a riposte to threatening processes. Springer, New York, pp 65-90

Burns KC (2012) Are introduced birds unimportant mutualists? A case study of frugivory in European blackbirds (Turdus merula). NZJ Ecol 36:171-176

Burns KC (2013) What causes size coupling in fruit-frugivore interaction webs? Ecology 94:295-300. doi: 10.1890/12-1161.1

Burns KC (2015) The color of plant reproduction: macroecological trade-offs between biotic signaling and abiotic tolerance. Front Ecol Evol 3:1-4. doi: $10.3389 /$ fevo.2015.00118

Butcher AR, Palethorpe HM, Grove DI (2002) Effects of sex and age on the susceptibility of C57BL/6J mice to infection with Brachylaima cribbi and the course of infection in NOD SCID mice. Parasitol Res 88:668-674. doi: 10.1007/s00436-002-0642-3

Calabrese JM, Brunner JL, Ostfeld RS (2011) Partitioning the aggregation of parasites on hosts into intrinsic and extrinsic components via an extended Poisson-gamma mixture model. PLoS One 6:e29215. doi:

10.1371/journal.pone.0029215

Cepeda-Cornejo V, Dirzo R (2010) Sex-related differences in reproductive allocation, growth, defense and herbivory in three dioecious neotropical palms. PLoS One 5:1-9. doi: 10.1371/journal.pone.0009824

Charles KE, Linklater WL (2013) Dietary breadth as a predictor of potential native avian - human conflict in urban landscapes. Wildl Res 40:482-489. doi: 10.1016/S0169-2046(97)00037-6 
Christe P, Glaizot O, Evanno G, et al. (2007) Host sex and ectoparasites choice: preference for, and higher survival on female hosts. J Anim Ecol 76:703-10. doi: 10.1111/j.1365-2656.2007.01255.x

Cibils AF, Swift DM, Hart RH (2003) Female-biased herbivory in fourwing saltbush browsed by cattle. J Range Manag 56:47. doi: 10.2307/4003880

Comas M, Ribas A (2013) Why are the prevalence and diversity of helminths in the endemic Pyrenean brook newt Calotriton asper (Amphibia , Salamandridae) so low ? J Helminthol 89:1-7. doi: 10.1017/S0022149X13000710

Cornelissen JHC, Lavorel S, Garnier E, et al. (2003) A handbook of protocols for standardised and easy measurement of plant functional traits worldwide. Aust $\mathrm{J}$ Bot 51:335-380. doi: 10.1071/BT02124

Cornelissen T, Stiling P (2005) Sex-biased herbivory: a meta-analysis of the effects of gender on plant-herbivore interactions. Oikos 111:488-500. doi: 10.1111/j.1600-0706.2005.14075.x

Cott H (1940) Adaptive colouration in animals. Oxford University Press

Cowan KM, Shutler D, Herman TB, Stewart DT (2007) Extreme male-biased infections of masked shrews by bladder nematodes. J Mammal 88:1539-1543. doi: 10.1644/06-MAMM-A-398R1.1

Cox RM, John-Alder HB (2007) Increased mite parasitism as a cost of testosterone in male striped plateau lizards Sceloporus virgatus. Funct Ecol 21:327-334. doi: 10.1111/j.1365-2435.2007.01251.x

Cuthill IC, Stevens M, Sheppard J, et al. (2005) Disruptive coloration and background pattern matching. Nature 434:72-74. doi: 10.1038/nature03312

Danell K, Elmqvist T, Ericson L, Salomonson A (1985) Sexuality in willows and preference by bark-eating voles: defence or not? Oikos 44:82-90. doi: $10.2307 / 3544047$

Danell K, Hjältén J, Ericson L, Elmqvist T (1991) Vole feeding on male and female willow shoots along a gradient of plant productivity. Oikos 62:145-152 
Daniels CW, Belosevic M (1994) Serum antibody responses by male and female C57BI/6 mice infected with Giardia muris. Clin Exp Immunol 97:424-9. doi: 10.1111/j.1365-2249.1994.tb06105.x

Daniels RR, Beltran S, Poulin R, Lagrue C (2013) Do parasites adopt different strategies in different intermediate hosts? Host size, not host species, influences Coitocaecum parvum (Trematoda) life history strategy, size and egg production. Parasitology 140:275-83. doi: 10.1017/S0031182012001564

Dare OK, Forbes MR (2009) Patterns of infection by lungworms, Rhabdias ranae and Haematoloechus spp., in northern leopard frogs: a relationship between sex and parasitism. J Parasitol 95:275-80. doi: 10.1645/GE-1713.1

Darwin C (1858) On the origin of species by means of natural selection. Muray, London

Daugherty C, Gibbs G, Hitchmough R (1993) Mega-island or micro-continent? New Zealand and its fauna. Trends Ecol Evol 8:437-442.

Davies OR, Junker K, Jansen R, et al. (2008) Age- and sex-based variation in helminth infection of helmeted guineafowl (Numida meleagris) with comments on Swainson's spurfowl (Pternistis swainsonii) and Orange River francolin (Scleroptila levaillantoides). South African J Wild Res 38:163-170. doi: 10.3957/0379-4369-38.2.163

Davis MB, Shaw RG (2001) Range shifts and adaptive responses to Quaternary climate change. Science 292:673-679. doi: 10.1126/science.292.5517.673

Davis T, Hofstetter R (2014) Allometry of phloem thickness and resin flow and their relation to tree chemotype in a southwestern ponderosa pine forest. For Sci $60: 1-5$

Dawkins R, Krebs JR (1979) Arms races between and within species. Proc R Soc Lond B Biol Sci 205:489-511

Dawson J, Lucas R (2011) New Zealand's Native Trees. Craig Potton Publishing, Nelson 
De Souza EM, Rivera M, Araujo-Jorge TC, De Castro SL (2001) Modulation induced by estradiol in the acute phase of Trypanosoma cruzi infection in mice. Parasitol Res 87:513-520. doi: 10.1007/s004360100376

Deslauriers A, Rossi S, Anfodillo T, Saracino A (2008) Cambial phenology, wood formation and temperature thresholds in two contrasting years at high altitude in southern Italy. Tree Physiol 28:863-871. doi: 10.1093/treephys/28.6.863

Dezfuli BS, Volponi S, Beltrami I, Poulin R (2002) Intra- and interspecific densitydependent effects on growth in helminth parasites of the cormorant, Phalacrocorax carbo sinensis. Parasitology 124:537-44.

Diaz M, Alonso CL (2003) Wood mouse Apodemus sylvaticus winter food supply: density, condition, breeding, and parasites. Ecology 84:2680-2691

Dick CW, Gannon MR, Little WE, Patrick MJ (2003) Ectoparasite associations of bats from central Pennsylvania. J Med Entomol 40:813-819. doi: $10.1603 / 0022-2585-40.6 .813$

Do Prado J, De Paula Leal M, Anselmo-Franci J a., et al. (1998) Influence of female gonadal hormones on the parasitemia of female Calomys callosus infected with the "Y" strain of Trypanosoma cruzi. Parasitol Res 84:100-105. doi: $10.1007 / \mathrm{s} 004360050364$

Dobson A, Lafferty KD, Kuris AM, et al. (2008) Colloquium paper: homage to Linnaeus: how many parasites? How many hosts? Proc Natl Acad Sci USA 105:11482-11489. doi: 10.1073/pnas.0803232105

Douglas AE (2006) Phloem-sap feeding by animals: problems and solutions. J Exp Bot 57:747-54. doi: 10.1093/jxb/erj067

Duan J, Larson K, Watt T, et al. (2013) Effects of host plant and larval density on intraspecific competition in larvae of the emerald ash borer (Coleoptera: Buprestidae). Environ Entomol 42:1193-1200

Duerr HP, Dietz K, Schulz-Key H, et al. (2004) The relationships between the burden of adult parasites, host age and the microfilarial density in human onchocerciasis. Int J Parasitol 34:463-473. doi: 10.1016/j.ijpara.2003.11.008 
Eberhardt L (2000) Use and selection of sap trees by Yellow-bellied sapsuckers. Auk $117: 41-51$

Edmunds M (1990) The evolution of cryptic colouration. In: Evans DL, Schmidt JO (eds) Insect defenses: adaptive mechanisms and strategies of prey and predators. State University of New York Press, Albany, pp 3-18

Elmqvist T, Ericson L, Danell K, Salomonson A (1988) Latitudinal sex ratio variation in willows, Salix spp., and gradients in vole herbivory. Oikos 51:259-266

Elmqvist T, Gardfjell H (1988) Differences in response to defoliation between males and females of Silene dioica. Oecologia 77:225-230

Endler JA. (1978) A predator's view of animal color patterns. In: Hecht MK, Steere WC, Wallace B (eds) Evolutionary Biology, 11th edition Plenum Press, New York and London, pp 319-364

Endler JA, Mielke PW (2005) Comparing entire colour patterns as birds see them. Biol J Linn Soc 86:405-431. doi: 10.1111/j.1095-8312.2005.00540.x

Fadzly N, Jack C, Schaefer HM, Burns KC (2009) Ontogenetic colour changes in an insular tree species: signalling to extinct browsing birds? New Phytol 184:495501. doi: 10.1111/j.1469-8137.2009.02926.x

Feller I, Mathis W (1997) Primary herbivory by wood-boring insects along an architectural gradient of Rhizophora mangle. Biotropica 29:440-451

Feller IC (2002) The role of herbivory by wood-boring insects in mangrove ecosystems in Belize. Oikos 97:167-176. doi: 10.1034/j.16000706.2002.970202.x

Feller IC, Hiroshi K, Tanner CE, Whigham DF (2002) Sex-biased herbivory in jack-inthe-pulpit (Arisaema triphyllum) by a specialist thrips (Heterothrips arisaemae). In: Marulla R, Mound L (eds) Thrips and tospoviruses: proceedings of the $7^{\text {th }}$ international sympoisum on Thysanoptera. Australian National Insect Collection, Canberra, pp 163-172 
Fernandes FR, Cruz LD, Linhares AX (2012) Effects of sex and locality on the abundance of lice on the wild rodent Oligoryzomys nigripes. Parasitol Res 111:1701-1706. doi: 10.1007/s00436-012-3009-4

Ferrari N, Rosà R, Pugliese a., Hudson PJ (2007) The role of sex in parasite dynamics: model simulations on transmission of Heligmosomoides polygyrus in populations of yellow-necked mice, Apodemus flavicollis. Int J Parasitol 37:341-349. doi: 10.1016/j.jpara.2006.10.015

Folstad I, Nilssen AC, Halvorsen O, Andersen J (1989) Why do male reindeer (Rangifer $t$. tarandus) have higher abundance of 2nd and 3rd instar larvae of Hypoderma tarandi than females. Oikos 55:87-92

Forsman A, Appelquist S (1998) Visual predators impose correlational selection on prey color pattern and behavior. Behav Ecol 9:409-413. doi: 10.1093/beheco/9.4.409

Fourie LJ, Horak IG, van den Heever JJ (1992) The relative importance of rock elephant shrews Elephantulus myurus and Namaqua rock mice Aethomys namaquensis for economically important ticks. South African J Zool 27:108114. doi: $10.1080 / 02541858.1992 .11448270$

Friman VP, Buckling A (2013) Effects of predation on real-time host-parasite coevolutionary dynamics. Ecol Lett 16:39-46. doi: 10.1111/ele.12010

Funk DJ, Bernays EA (2001) Geographic variation in host specificity reveals host range evolution in Uroleucon ambrosiae aphids. Ecology 82:726-739

Gannon MR, Willig MR (1995) Ecology of ectoparasites from tropical bats. Environ Entomol 24:1495-1503

Gilburn AS, Stewart KM, Edward DA (2009) Sex-biased phoretic mite load on two seaweed flies: Coelopa frigida and Coelopa pilipes. Environ Entomol 38:16081612. doi: $10.1603 / 022.038 .0612$

Goble FC, Konopka EA (1973) Sex as a factor in infectous disease. Trans NY Acad Sci 35:325-346 
Godfrey SS, Moore JA, Nelson NJ, Bull CM (2010) Social network structure and parasite infection patterns in a territorial reptile, the tuatara (Sphenodon punctatus). Int J Parasitol 40:1575-1585. doi: 10.1016/j.ijpara.2010.06.002

Goedknegt A, Welsh J, Thieltges D (2012) Parasites as Prey. In: Encyclopedia of Life Sciences. John Wiley \& Sons, Ltd, Chichester. doi: 10.1002/9780470015902.a0023604

Gómez A, Nichols E (2013) Neglected wild life: parasitic biodiversity as a conservation target. Int J Parasitol Parasites Wildl 2:222-7. doi: 10.1016/j.ijppaw.2013.07.002

Gorrell JC, Schulte-Hostedde Al (2008) Patterns of parasitism and body size in red squirrels (Tamiasciurus hudsonicus). Can J Zool 86:99-107. doi: 10.1139/Z07123

Gotthard K, Margraf N, Rahier M (2004) Geographic variation in oviposition choice of a leaf beetle: the relationship between host plant ranking, specificity, and motivation. Entomol Exp Appl 110:217-224. doi: 10.1111/j.00138703.2004.00138.x

Graff P, Rositano F, Aguiar MR (2013) Changes in sex ratios of a dioecious grass with grazing intensity: the interplay between gender traits, neighbour interactions and spatial patterns. J Ecol 101:1146-1157. doi: 10.1111/13652745.12114

Grant JB (2007) Ontogenetic colour change and the evolution of aposematism: A case study in panic moth caterpillars. J Anim Ecol 76:439-447. doi: 10.1111/j.1365-2656.2007.01216.x

Graetz RD (1978) The influence of grazing by sheep on the structure of a saltbush (Atriplex vesicaria Hew. ex Benth.) population. Aus Ran J 1,2:117-125

Green C (2005) Using artificial refuges to translocate and establish Auckland tree weta Hemideina thoracica on Korapuki Island, New Zealand. Conserv Evid 2:94-95

Greenleaf S, Williams N, Winfree R, Kremen C (2007) Bee foraging ranges and their relationship to body size. Oecologia 153:589-596. doi: 10.1007/s00442-007 
Grehan J (1987) Life cycle of the wood-borer Aenetus virescens (Lepidoptera: Hepialidae). New Zeal J Zool 14:209-217

Grehan JR (1984) The host range of Aenetus virescens (Lepidoptera: Hepialidae) and its evolution. New Zeal Entomol 8:52 - 61

Grehan JR (1981) Morphological changes in the three-phase development of Aenetus virescens larvae (Lepidoptera: Hepialidae). New Zeal J Zool 8:505514

Grehan JR (1983) Larval establishment behaviour of the borer Aenetus virescens (Lepidoptera: Hepialidae) in live trees. New Zeal Entomol 7:413-417

Gričar J (2012) Cambial cell production and structure of xylem and phloem as an Indicator of tree vitality: a review. In: Diez Casero JJ, Garcia JM (eds) Sustainable Forest Management - Current Research. InTech, Rijeka, pp 111134

Grutter A, Poulin R (1998) Intraspecific and interspecific relationships between host size and the abundance of parasitic larval gnathiid isopods on coral reef fishes. Mar Ecol Prog Ser 164:263-271. doi: 10.3354/meps164263

Grzybek M, Bajer A, Behnke-Borowczyk J, et al. (2014) Female host sex-biased parasitism with the rodent stomach nematode Mastophorus muris in wild bank voles (Myodes glareolus). Parasitol Res 114:523-533. doi: 10.1007/s00436014-4214-0

Halvorsen O, Skorping A, Hansen K (1985) Seasonal cycles in the output of first stage larvae of the nematode Elaphostongylus rangiferi from Reindeer, Rangifer tarandus tarandus. Polar Biol 5:49-54

Hanks LM, Paine TD, Millar JG, et al. (1999) Water relations of host trees and resistance to the phloem-boring beetle Phoracantha semipunctata F. (Coleoptera: Cerambycidae). Oecologia 119:400-407. doi: $10.1007 / s 004420050801$

Hansson BS (1995) Olfaction in Lepidoptera. Experientia 51:1003-1027 
Harrison A, Scantlebury M, Montgomery WI (2010) Body mass and sex-biased parasitism in wood mice Apodemus sylvaticus. Oikos 119:1099-1104. doi: 10.1111/j.1600-0706.2009.18072.x

Haukioja E, Koricheva J (2000) Tolerance to herbivory in woody vs. herbaceous plants. Evol Ecol 14:551-562

Hillegass MA, Waterman JM, Roth JD (2008) The influence of sex and sociality on parasite loads in an African ground squirrel. Behav Ecol 19:1006-1011. doi: 10.1093/beheco/arn070

Hjältén J (1992) Plant sex and hare feeding preferences. Oecologia 89:253-256. doi: 10.1007/BF00317225

Hlaka L, Mukaratirwa S, Kamau J (2015) Host-sex influences the establishment of Trichinella zimbabwensis in Sprague-Dawley rats. Int J Appl Res Vet Med 13:141-146

Hoby S, Schwarzenberger F, Doherr MG, et al. (2006) Steroid hormone related male biased parasitism in chamois, Rupicapra rupicapra rupicapra. Vet Parasitol 138:337-348. doi: 10.1016/j.vetpar.2006.01.028

Hockley FA, Williams CF, Reading AJ, et al. (2011) Parasite fauna of introduced pumpkinseed fish Lepomis gibbosus: first British record of Onchocleidus dispar (Monogenea). Dis Aquat Organ 97:65-73. doi: 10.3354/dao02402

Högberg P, Nordgren A, Buchmann N, et al. (2001) Large-scale forest girdling shows that current photosynthesis drives soil respiration. Nature 411:789-92. doi: $10.1038 / 35081058$

Hölttä T, Kurppa M, Nikinmaa E (2013) Scaling of xylem and phloem transport capacity and resource usage with tree size. Front Plant Sci 4:496. doi: 10.3389/fpls.2013.00496

Hudson R, Trillmich F (2008) Sibling competition and cooperation in mammals: challenges, developments and prospects. Behav Ecol Sociobiol 62:299-307. doi: 10.1007/s00265-007-0417-z 
Hultgren KM, Stachowicz JJ (2008) Alternative camouflage strategies mediate predation risk among closely related co-occurring kelp crabs. Oecologia 155:519-528. doi: 10.1007/s00442-007-0926-5

Hurd H (2001) Host fecundity reduction: a strategy for damage limitation? Trends Parasitol 17:363-368. doi: 10.1016/S1471-4922(01)01927-4

Ikeda K (1979) Consumption and food utilization by individual larvae and the population of a wood borer Phymatodes maaki Kraatz (Coleoptera: Cerambycidae). Oecologia 40:287-298

Imasuen AA, Aisien MSO, Weldon C, et al. (2011) Occurrence of Batrachochytrium dendrobatidis in amphibian populations of Okomu national park, Nigeria. Herpetol Rev 42:379-382

Isomursu M, Rätti O, Helle P, Hollmén T (2006) Sex and age influence intestinal parasite burden in three boreal grouse species. J Avian Biol 37:516-522. doi: 10.1111/j.2006.0908-8857.03838.x

Jian X, Sen L, Hui-Qin Q, et al. (2003) Necator americanus: Maintenance through one hundred generations in golden hamsters (Mesocricetus auratus). I. Host sex-associated differences in hookworm burden and fecundity. Exp Parasitol 104:62-66. doi: 10.1016/S0014-4894(03)00094-8

Jing SW, Coley PD (1990) Dioecy and herbivory: the effect of growth rate on plant defense in Acer Negundo. Oikos 58:369-377

Johnson PTJ, Dobson A, Lafferty KD, et al. (2010) When parasites become prey: ecological and epidemiological significance of eating parasites. Trends Ecol Evol 25:362-71. doi: 10.1016/j.tree.2010.01.005

Jones J, Siefferman L (2014) Agonistic behaviors between chestnut-sided (Setophaga pensy/vanica) and golden-winged (Vermivora chrysoptera) warblers are unlikely a result of plumage misidentification. Wilson $\mathrm{J}$ Ornithol 126:708-716. doi: http://dx.doi.org/10.1676/14-028.1

Joy JE, Bunten CA (1997) Cosmocercoides variabilis (Nematoda: Cosmocercoidea) populations in the Eastern American toad, Bufo a. americanus (Salienta: 
Bufonidae), from western West Virginia. J Helminthol Soc Washingt 64:102105.

Kamis AB, Ibrahim JB (1989) Effects of testosterone on blood leukocytes in Plasmodium berghei-infected mice. Parasitol Res 75:611-613. doi: 10.1007/BF00930957

Kamis AB, Ahmad RA, Zoologi J (1992) Worm burden and leukocyte response in Angiostongylus malaysiensis-infected rats: the influence of testosterone. Parasitol Reasearch 78:388-391

Kaňuch P, Krištín A, Krištofík J (2005) Phenology, diet, and ectoparasites of Leisler's bat (Nyctalus leisleri) in the Western Carpathians (Slovakia). Acta Chiropterologica 7:249-257. doi: 10.3161/17335329(2005)7[249:PDAEOL]2.0.CO;2

Kaplan I, Denno RF (2007) Interspecific interactions in phytophagous insects revisited: a quantitative assessment of competition theory. Ecol Lett 10:977994. doi: 10.1111/j.1461-0248.2007.01093.x

Kavanagh PH, Lehnebach C a, Shea MJ, Burns KC (2011) Allometry of sexual size dimorphism in dioecious plants: do plants obey Rensch's rule? Am Nat 178:596-601. doi: 10.1086/662175

Keeley E, Grant J (1995) Allometric and environmental correlates of territory size in juvenile Atlantic salmon (Salmo salar). Can J Fish Aquat Sci 196:186-196

Kehr J (2006) Phloem sap proteins: their identities and potential roles in the interaction between plants and phloem-feeding insects. J Exp Bot 57:767-74. doi: $10.1093 / j x b / e r j 087$

Kelehear C, Brown GP, Shine R (2012) Size and sex matter: infection dynamics of an invading parasite (the pentastome Raillietiella frenatus) in an invading host (the cane toad Rhinella marina). Parasitology 139:1596-604. doi: $10.1017 /$ S0031182012000832

Kiffner C, Stanko M, Morand S, et al. (2013) Sex-biased parasitism is not universal: Evidence from rodent-flea associations from three biomes. Oecologia 173:1009-1022. doi: 10.1007/s00442-013-2664-1 
Kiffner C, Stanko M, Morand S, et al. (2014) Variable effects of host characteristics on species richness of flea infracommunities in rodents from three continents. Parasitol Res 113:2777-2788. doi: 10.1007/s00436-014-3937-2

King DA, Davies SJ, Tan S, Noor NSM (2006) The role of wood density and stem support costs in the growth and mortality of tropical trees. J Ecol 94:670-680. doi: 10.1111/j.1365-2745.2006.01112.x

Kingsolver JG, Shlichta JG, Ragland GJ, Massie KR (2006) Thermal reaction norms for caterpillar growth depend on diet. 703-715.

Klein SL (2004) Hormonal and immunological mechanisms mediating sex differences in parasite infection. Parasite Immunol 26:247-264. doi: 10.1111/j.0141-9838.2004.00710.x

Klein SL, Gamble HR, Nelson RJ (1999) Role of steroid hormones in Trichinella spiralis infection among voles. Am J Physiol 277:R1362-7.

Kolb TE, Guerard N, Hofstetter RW, Wagner MR (2006) Attack preference of Ips pini on Pinus ponderosa in northern Arizona: tree size and bole position. Agric For Entomol 8:295-303. doi: 10.1111/j.1461-9563.2006.00308.x

Kopelke JP, Amendt J, Schönrogge K (2003) Patterns of interspecific associations of stem gallers on willows. Divers Distrib 9:443-453. doi: 10.1046/j.14724642.2003.00037.x

Korpimaki E, Tolonen K, Bennet G (1995) Blood parasites, sexual selection and reproductive success of European kestrels. Ecoscience 2:335-343

Krasnov BR, Bordes F, Khokhlova IS, Morand S (2012) Gender-biased parasitism in small mammals: patterns, mechanisms, consequences. Mammalia 76:1-13. doi: 10.1515/mammalia-2011-0108

Krasnov BR, Khokhlova IS, Arakelyan MS, Degen AA (2005a) Is a starving host tastier? Reproduction in fleas parasitizing food-limited rodents. Funct Ecol 19:625-631. doi: 10.1111/j.1365-2435.2005.01015.x

Krasnov BR, Morand S, Hawlena H, et al. (2005b) Sex-biased parasitism, seasonality and sexual size dimorphism in desert rodents. Oecologia 146:209217. doi: $10.1007 / \mathrm{s} 00442-005-0189-y$ 
Krasnov BR, Poulin R, Shenbrot Gl, et al. (2004) Ectoparasitic "jacks-of-all-trades": relationship between abundance and host specificity in fleas (Siphonaptera) parasitic on small mammals. Am Nat 164:506-16. doi: 10.1086/423716

Krichbaum K, Perkins S, Gannon MR (2009) Host-parasite interactions of tropical bats in Puerto Rico. Acta Chiropterologica 11:157-162. doi: $10.3161 / 150811009 \times 465776$

Krischik VA, Denno RF (1990) Patterns of growth, reproduction, defense, and herbivory in the dioecious shrub Baccharis halimifolia (Compositae). Oecologia $83,2: 182-190$

Kührt U, Samietz J, Dorn S (2005) Thermoregulation behaviour in codling moth larvae. Physiol Entomol 30:54-61. doi: 10.1111/j.0307-6962.2005.00431.x

Kuris A, Blaustein A, Alio J (1980) Hosts as islands. Am Nat 116:570-586

Lafferty KD, Allesina S, Arim M, et al. (2008) Parasites in food webs: the ultimate missing links. Ecol Lett 11:533-46. doi: 10.1111/j.1461-0248.2008.01174.x

Lafferty KD, Dobson AP, Kuris AM (2006) Parasites dominate food web links. Proc Natl Acad Sci U S A 103:11211-6. doi: 10.1073/pnas.0604755103

Lagrue C, Poulin R (2008) Intra- and interspecific competition among helminth parasites: effects on Coitocaecum parvum life history strategy, size and fecundity. Int J Parasitol 38:1435-44. doi: 10.1016/j.ijpara.2008.04.006

Langmore NE, Hunt S, Kilner RM (2003) Escalation of a coevolutionary arms race through host rejection of brood parasitic young. Nature 422:157-60. doi: 10.1038 /nature 01460

Lareschi M (2006) The relationship of sex and ectoparasite infestation in the water rat Scapteromys aquaticus (Rodentia: Cricetidae) in La Plata, Argentina. Rev Biol Trop 54:673-679

Larralde C, Morales J, Terrazas I, et al. (1995) Sex hormone changes induced by the parasite lead to feminization of the male host in murine Taenia crassiceps cysticercosis. J Steroid Biochem Mol Biol 52:575-580. doi: 10.1016/09600760(95)00062-5 
Lester RJG (2012) Overdispersion in marine fish parasites. J Parasitol 98:718-21. doi: 10.1645/GE-3017.1

Levesque KR, Levesque KR, Fortin M, Mauffette Y (2002) Temperature and food quality effects on growth, consumption and post-ingestive utilization efficiencies of the forest tent caterpillar Malacosoma disstria (Lepidoptera: Lasiocampidae). Bull Entomol Res 92:127-136. doi: 10.1079/BER2002153

Liesenfeld O, Nguyen T a, Pharke C, Suzuki Y (2001) Importance of gender and sex hormones in regulation of susceptibility of the small intestine to peroral infection with Toxoplasma gondii tissue cysts. J Parasitol 87:1491-1493. doi: 10.1645/0022-3395(2001)087[1491:IOGASH]2.0.CO;2

Lindstedt S, Miller B, Buskirk S (1986) Home range, time, and body size in mammals. Ecology 67:413-418.

Lomolino M (1990) The target area hypothesis: the influence of island area on immigration rates of non-volant mammals. Oikos 57:297-300.

Lootvoet A, Blanchet S, Gevrey M, et al. (2013) Patterns and processes of alternative host use in a generalist parasite: insights from a natural hostparasite interaction. Funct Ecol 27:1403-1414. doi: 10.1111/1365-2435.12140

Lovett Doust J, Lovett Doust L (1985) Sex ratios, clonal growth and herbivory in Rumex acetosella: Studies on Plant Demography, 327 - 341

Lozano G (1991) Optimal foraging theory : a possible role for parasites. Oikos 60:391-395.

Lučan RK (2006) Relationships between the parasitic mite Spinturnix andegavinus (Acari: Spinturnicidae) and its bat host, Myotis daubentonii (Chiroptera: Vespertilionidae): Seasonal, sex- and age-related variation in infestation and possible impact of the parasite on the. Folia Parasitol (Praha) 53:147-152. doi: 10.14411/fp.2006.019

Lucero García-García P, Cano-Santana Z (2015) Nutritional ecology, growth and density of Acronyctodes mexicanaria (Lepidoptera: Geometridae) on a dioecious plant Buddleja cordata (Scrophulariaceae). Rev Mex Biodivers 86:172-177. doi: 10.7550/rmb.44382 
Luong LT, Platzer EG, Zuk M, Giblin-Davis RM (2000) Venereal worms: sexually transmitted nematodes in the decorated cricket. J Parasitol 86:471-477. doi: 10.1645/0022-3395(2000)086

Macara G (2014) New Zealand Annual Climate Summary. NIWA.

MacArthur RH, Wilson EO (1967) The Theory of Island Biogeography. Princeton University Press

Maia R, Eliason C (2013) pavo: an R package for the analysis, visualization and organization of spectral data. Methods Ecol Evol 4:906-913

Maldonado-López Y, Cuevas-Reyes P, Sánchez-Montoya G, et al. (2014) Growth, plant quality and leaf damage patterns in a dioecious tree species: is gender important? Arthropod Plant Interact 8:241-251. doi: 10.1007/s11829-014-93143

Mallet J, Joron M (1999) Evolution of diversity in warning color and mimicry: polymorphisms, shifting balance, and speciation. Annu Rev Ecol Syst 30:201233.

Marguerite M, Gallissot MC, Diagne M, et al. (1999) Cellular immune responses of a Senegalese community recently exposed to Schistosoma mansoni: Correlations of infection level with age and inflammatory cytokine production by soluble egg antigen-specific cells. Trop Med Int Heal 4:530-543. doi: 10.1046/j.1365-3156.1999.00443.x

Martinez-Trinidad T, Watson WT, Arnold MA, et al. (2010) Comparing various techniques to measure tree vitality of live oaks. Urban For Urban Green 9:199_ 203. doi: 10.1016/j.ufug.2010.02.003

Matter S, Roland J (2003) The effects of isolation, habitat area and resources on the abundance, density and movement of the butterfly Parnassius smintheus. Am Midl Nat 150:26-36

Matthee S, McGeoch M, Krasnov B (2010) Parasite-specific variation and the extent of male-biased parasitism; an example with a South African rodent and ectoparasitic arthropods. Parasitology 137:651-660. doi: $10.1017 /$ S0031182009991338 
McCoy KD (2003) Sympatric speciation in parasites - what is sympatry? Trends Parasitol 19:400-404. doi: 10.1016/S1471-4922(03)00194-6

McCurdy DG, Shutler D, Mullie A, Forbes MR (1998) Sex-biased parasitism of avian hosts: Relations to blood parasite taxon and mating system. Oikos 82:303-312. doi: $10.2307 / 3546970$

Mega NO (2014) The adult body size variation of Dryas iulia (Lepidoptera, Nymphalidae, Heliconiinae) in different populations is more influenced by temperature variation than by host-plant availability during the seasons. Entomol Sci 17:376-387. doi: 10.1111/ens.12077

Merilaita S, Dimitrova M (2014) Accuracy of background matching and prey detection: Predation by blue tits indicates intense selection for highly matching prey colour pattern. Funct Ecol 28:1208-1215. doi: 10.1111/1365-2435.12248

Merilaita S, Lind J (2005) Background-matching and disruptive coloration, and the evolution of cryptic coloration. Proc Biol Sci 272:665-70. doi: $10.1098 /$ rspb.2004.3000

Mock B a., Nacy C a. (1988) Hormonal modulation of sex differences in resistance to Leishmania major systemic infections. Infect Immun 56:3316-3319

Molineaux L, Gramiccia G (1980) The Garki project: research on the epidemiology and control of malaria in the Sudan savanna of West Africa. The World Health Organization, Geneva

Morales-Montor AJ, Chavarria A, de León MA, et al. (2004) Host gender in parasitic infections of mammals: an evaluation of the female host supremacy paradigm. J Parasitol 90:531-546. doi: 10.1645/GE-113R3

Morales-Montor J, Baig S, Hallal-Calleros C, Damian RT (2002) Taenia crassiceps: Androgen reconstitution of the host leads to protection during cysticercosis. Exp Parasitol 100:209-216. doi: 10.1016/S0014-4894(02)00028-0

Morand S, De Bellocq JG, Stanko M, Miklisová D (2004) Is sex-biased ectoparasitism related to sexual size dimorphism in small mammals of Central Europe? Parasitology 129:505-510. doi: 10.1017/S0031182004005840 
Moravvej G, Hamidi K, Nourani L (2016) Relationship between the sex and age of Mus musculus (Rodentia: Muridae) with ectoparasites prevalence in northeast of Iran. Persian J Acarol 5:51-62

Muilenburg VL, Phelan PL, Bonello P, et al. (2013) Characterization of wound responses of stems of paper birch (Betula papyrifera) and European white birch (Betula pendula). Trees - Struct Funct 27:851-863. doi: 10.1007/s00468-0130839-3

Muñoz L, Aguilera M, Casanueva ME (2003) Prevalencia e intensidad de ectoparasitos asociados a Tadarida brasiliensis (Geoffroy Saint-Hilaire, 1824) (Chiroptera: Molossidae) En Concepcion. Gayana (Concepción) 67:1-8. doi: 10.4067/S0717-65382003000100001

Nakazawa M, Fantappie MR, Freeman GL, et al. (1997) Schistosoma mansoni: susceptibility differences between male and female mice can be mediated by testosterone during early infection. Exp Parasitol 85:233-40. doi: 10.1006/expr.1997.4148

Nentwig W (1985) A tropical caterpillar that mimics faeces leaves and a snake (Lepidoptera: Oxytenidae: Oxytenis naemia). J Res Lepidoptera 24:136-141.

Neuhäuser M, Kotzmann J, Walier M, Poulin R (2010) The comparison of mean crowding between two groups. J Parasitol 96:477-81. doi: 10.1645/GE-2177.1

Nichols E, Gómez A (2011) Conservation education needs more parasites. Biol Conserv 144:937-941. doi: 10.1016/j.biocon.2010.10.025

Nicolai V (1986) The bark of trees: thermal properties, microclimate and fauna. Oecologia 69:148-160. doi: 10.1007/BF00399052

Nylin S, Gamberale-Stille G, Tullberg BS (2001) Ontogeny of defense and adaptive coloration in larvae of the comma butterfly, Polygonia c-album (Nymphalidae). J Lepid Soc 55:69-73

Olsson-Pons S, Clark NJ, Ishtiaq F, Clegg SM (2015) Differences in host species relationships and biogeographic influences produce contrasting patterns of prevalence, community composition and genetic structure in two genera of 
avian malaria parasites in southern Melanesia. J Anim Ecol 84:985-998. doi: $10.1111 / 1365-2656.12354$

Patterson BD, Dick CW, Dittmar K (2008) Parasitism by bat flies (Diptera: Streblidae) on neotropical bats: effects of host body size, distribution, and abundance. Parasitol Res 103:1091-1100. doi: 10.1007/s00436-008-1097-y

Paling JE (1965) The population dynamics of the monogenean gill parasite Discocotyle sagittata Leuckart on Windermere trout, Salmo trutta, L. Parasitology. 55,4:667-694

Pedersen AB, Altizer S, Poss M, et al. (2005) Patterns of host specificity and transmission among parasites of wild primates. Int J Parasitol 35:647-57. doi: 10.1016/j.ijpara.2005.01.005

Perez-Orella C, Schulte-Hostedde Al (2005) Effects of sex and body size on ectoparasite loads in the northern flying squirrel (Glaucomys sabrinus). Can J Zool 83:1381-1385. doi: 10.1139/Z05-126

Plamping K, Haigh M, Cullis MJ, Jenkins RE (2009) Evaluation of cambial electrical resistance for the appraisal of tree vitality on reclaimed coal lands. Int J Mining, Reclam Environ 23:21-32. doi: 10.1080/17480930801999419

Polhemus DA (1988) Intersexual variation in densities of plant bugs (Hemiptera: Miridae) on Juniperus scopulorum. Ann. Ent. Soc. Am 81,5:742-747

Pompon J, Quiring D, Goyer C, et al. (2011) A phloem-sap feeder mixes phloem and xylem sap to regulate osmotic potential. J Insect Physiol 57:1317-22. doi: 10.1016/j.jinsphys.2011.06.007

Poorter L, McDonald I, Alarcón A, et al. (2010) The importance of wood traits and hydraulic conductance for the performance and life history strategies of 42 rainforest tree species. New Phytol 185:481-92. doi: 10.1111/j.14698137.2009.03092.x

Poulin R (2000) Variation in the intraspecific relationship between fish length and intensity of parasitic infection: biological and statistical causes. J Fish Biol 123137 
Poulin R (1999) Body size vs abundance among parasite species: positive relationships? Ecography (Cop) 22:246-250

Poulin R (2013) Explaining variability in parasite aggregation levels among host samples. Parasitology 140:541-6. doi: 10.1017/S0031182012002053

Poulin R (2007) Evolutionary ecology of parasites, 2nd ed. Princeton University Press

Poulin R (2005) Evolutionary trends in body size of parasitic flatworms. Biol J Linn Soc 85:181-189. doi: 10.1111/j.1095-8312.2005.00481.x

Poulin R, Forbes MR (2011) Meta-analysis and research on host-parasite interactions: past and future. Evol Ecol 26:1169-1185. doi: 10.1007/s10682011-9544-0

Poulin R, Keeney DB (2008) Host specificity under molecular and experimental scrutiny. Trends Parasitol 24:24-28. doi: 10.1016/j.pt.2007.10.002

Poulin R, Krasnov BR, Morand S (2006) Patterns of host specificity in parasites exploiting small mammals. Micromammals Macroparasites From Evol Ecol to Manag 233-256. doi: 10.1007/978-4-431-36025-4_13

Poulin R, Krasnov BR, Mouillot D (2011) Host specificity in phylogenetic and geographic space. Trends Parasitol 27:355-61. doi: 10.1016/j.pt.2011.05.003

Poulin R, Latham A (2003) Effects of initial (larval) size and host body temperature on growth in trematodes. Can J Zool 581:574-581. doi: 10.1139/Z03-039

Poulin R, Morand S (2000) Parasite body size and interspecific variation in levels of aggregation among nematodes. J Parasitol 86:642-7. doi: 10.1645/00223395(2000)086[0642:PBSAIV]2.0.CO;2

Poulin R, Morand S (2004) Parsite Biodiversity. Smithsonian Books

Poulin R, Wise M, Moore J (2003) A comparative analysis of adult body size and its correlates in acanthocephalan parasites. Int J Parasitol 33:799-805. doi: 10.1016/S0020-7519(03)00108-5

Poulton EB (1890) The colours of animals: their meaning and use, especially considered in the case of insects. New York: Appleton 
R Core Team (2015) R: A Language and Environment for Statistical Computing.

Randhawa HS, Poulin R (2009) Determinants and consequences of interspecific body size variation in tetraphyllidean tapeworms. Oecologia 161:759-69. doi: 10.1007/s00442-009-1410-1

Rankin L, Borden J (1991) Competitive interactions between the mountain pine beetle and the pine engraver in lodgepole pine. Can J For Res 21:1029-1036.

Rasband WS (2014) ImageJ. National Institutes of Health, Bethesda

Reimchen TE, Nosil P (2001) Ecological causes of sex-biased parasitism in threespine stickleback. Biol J Linn Soc 73:51-63. doi: 10.1006/bijl.2001.0523

Richner H, Christe P, Oppliger A (1995) Paternal investment affects prevalence of malaria. Proc Natl Acad Sci USA 92:1192-4. doi: 10.1073/pnas.92.4.1192

Roberts CW, Cruickshank SM, Alexander J (1995) Sex-determined resistance to Toxoplasma gondii is associated with temporal differences in cytokine production. Infect Immun 63:2549-2555

Robinson SA, Forbes MR, Hebert CE, Daniel McLaughlin J (2008) Male-biased parasitism by common helminths is not explained by sex differences in body size or spleen mass of breeding cormorants Phalacrocorax auritus. J Avian Biol 39:272-276. doi: 10.1111/j.0908-8857.2008.04340.x

Rohde K (1993) Ecology of marine parasites: an introduction to marine parasites, 2nd ed. Oxford University Press

Rossin A, Malizia Al (2002) Relationship between helminth parasites and demographic attributes of a population of the subterranean rodent Ctenomys talarum (Rodentla: Octodontidae). J Parasitol 88:1268-1270. doi: 10.1645/0022-3395(2002)088[1268:RBHPAD]2.0.CO;2

Rossin MA, Malizia AI, Timi JT, Poulin R (2010) Parasitism underground: determinants of helminth infections in two species of subterranean rodents (Octodontidae). Parasitology 137:1569-1575. doi:

$10.1017 /$ S0031182010000351 
Rowland HM, Cuthill IC, Harvey IF, et al. (2008) Can't tell the caterpillars from the trees: countershading enhances survival in a woodland. Proc Biol Sci 275:2539-45. doi: 10.1098/rspb.2008.0812

Ruf C, Fiedler K (2002) Tent-based thermoregulation in social caterpillars of Eriogaster lanestris (Lepidoptera: Lasiocampidae): behavioral mechanisms and physical features of the tent. J Therm Biol 27:493-501. doi: 10.1016/S03064565(02)00022-0

Ruxton G, Sherratt T, Speed M (2004) Avoiding attack: the evolutionary ecology of crypsis, warning signals, and mimicry. Oxford University Press, Oxford

Sadras VO, Denison RF (2009) Do plant parts compete for resources? An evolutionary viewpoint. New Phytol 183:565-574. doi: 10.1111/j.14698137.2009.02848.x

Saldanha I, Leung TLF, Poulin R (2009) Causes of intraspecific variation in body size among trematode metacercariae. J Helminthol 83:289-93. doi: $10.1017 / \mathrm{S} 0022149 \times 09224175$

Salkeld DJ, Schwarzkopf L (2005) Epizootiology of blood parasites in an Australian lizard: A mark-recapture study of a natural population. Int J Parasitol 35:11-18. doi: 10.1016/j.jpara.2004.09.005

Sanchez A, Devevey G, Bize P (2011) Female-biased infection and transmission of the gastrointestinal nematode Trichuris arvicolae infecting the common vole, Microtus arvalis. Int J Parasitol 41:1397-1402. doi: 10.1016/j.ijpara.2011.09.004

Santini NS, Schmitz N, Lovelock CE (2012) Variation in wood density and anatomy in a widespread mangrove species. Trees 26:1555-1563. doi: 10.1007/s00468012-0729-0

Scantlebury M, Maher McWilliams M, Marks NJ, et al. (2010) Effects of life-history traits on parasite load in grey squirrels. J Zool 282:246-255. doi: 10.1111/j.1469-7998.2010.00734.x

Schaefer HM, Ruxton GD (2009) Deception in plants: mimicry or perceptual exploitation? Trends Ecol Evol 24:676-85. doi: 10.1016/j.tree.2009.06.006 
Schall JJ, Marghoob AB (1995) Prevalence of a Malarial Parasite Over Time and Space: Plasmodium mexicanum in its vertebrate host, the western fence lizard Sceloporus occidentalis. J Anim Ecol 64:177-185. doi: 10.2307/5753

Schall JJ, Pearson AR, Perkins SL (2000a) Prevalence of malaria parasites (Plasmodium floridense and Plasmodium azurophilum ) Infecting a Puerto Rican Lizard (Anolis Gundlachi): a Nine-Year Study. 86:511-515

Schall JJ, Prendeville HR, Hanley KA, Herpetology J (2000b) Prevalence of the tick, Ixodes pacificus, on western fence lizards, Sceloporus occidentalis: trends by gender, size, season, site, and mite infestation. J Herpetol 34:160-163

Schmidt V, Schaefer HM, Winkler H (2004) Conspicuousness, not colour as foraging cue in plant-animal signalling. Oikos 106:551-557

Sears BF, Schlunk AD, Rohr JR (2012) Do parasitic trematode cercariae demonstrate a preference for susceptible host species? PLoS One 7:e51012. doi: 10.1371/journal.pone.0051012

Senlik B, Cirak VY, Girisgin O, Akyol C V (2011) Helminth infections of wild boars (Sus scrofa) in the Bursa province of Turkey. J Helminthol 85:404-8. doi: 10.1017/S0022149X1000074X

Shaanker RU, Ganeshaiah KN, Bawa KS (1988) Parent-offspring Conflict, Sibling Rivalry, and Brood Size Patterns in Plants. Annu Rev Ecol Syst 19:177-205. doi: 10.1146/annurev.es.19.110188.001141

Shaw DJ, Grenfell BT, Dobson a P (1998) Patterns of macroparasite aggregation in wildlife host populations. Parasitology 117 (Pt 6):597-610

Sheridan LAD, Poulin R, Ward DF, Zuk M (2000) Sex differences in parasitic infections among arthropod hosts: is there a male bias? Oikos 88:327-334. doi: 10.1034/j.1600-0706.2000.880211.x

Siddiqi A, Cronin TW, Loew ER, et al. (2004) Interspecific and intraspecific views of color signals in the strawberry poison frog Dendrobates pumilio. J Exp Biol 207:2471-85. doi: 10.1242/jeb.01047

Soliman S, Marzouk AS, Main AJ, Montasser AA (2001) Effect of sex, size, and age of commensal rat hosts on the infestation parameters of their ectoparasites in a 
rural area of Egypt. J Parasitol 87:1308-1316. doi: 10.1645/0022-

3395(2001)087[1308:EOSSAA]2.0.CO;2

Southwood T, Kennedy C (1983) Trees as islands. Oikos 41:359-371

Speights JR, Conway WC (2010) Habitat and host tree selection by wintering in East Texas sapsuckers. Am Midl Nat 164:107-118

Spratt DM, Haycock P, Boyden JM, Nicholas WL (2002) Aspects of the life history of Muspicea borreli (Nematoda: Muspiceidae), parasite of the house mouse (Mus domesticus) in Australia. Parasite 9:199-205. doi:

10.1051/parasite/2002093199

Stachowicz JJ, Hay ME, Ecology S, Mar N (2012) Reducing predation through chemically mediated camouflage: indirect effects of plant defenses on herbivores. Ecology 80:495-509

Stark HE, Miles VI (1962) Ecological Studies of Wild Rodent Plague in the San Francisco Bay Area of California. VI. The Relative Abundance of Certain Flea Species and their Host Relationships on Coexisting Wild and Domestic Rodents. Amer J Trop Med Hyg 11:4, 525 -534

Stevens M, Merilaita S (2009) Animal camouflage: current issues and new perspectives. Philos Trans R Soc Lond B Biol Sci 364:423-7. doi: 10.1098/rstb.2008.0217

Stevens M, Tevens MS (2015) Anti-predator coloration and behaviour : a longstanding topic with many outstanding questions. Curr Zool 61:702-707

Stewart A, Komers PE (2012) Testing the ideal free distribution hypothesis: moose response to changes in habitat amount. ISRN Ecol 2012:1-8. doi: $10.5402 / 2012 / 945209$

Stoddard MC, Prum RO (2008) Evolution of avian plumage color in a tetrahedral color space: a phylogenetic analysis of new world buntings. Am Nat 171:75576. doi: $10.1086 / 587526$

Stoddard MC, Stevens M (2011) Avian vision and the evolution of egg color mimicry in the common cuckoo. Evolution 65:2004-2013. doi: 10.1111/j.15585646.2011.01262.x 
Stowe KA, Marquis RJ, Hochwender CG, Simms EL (2000) The evolutionary ecology of tolerance to consumer damage. Ann Rev Ecol Syst 31:565-595

Stuart-Fox D, Moussalli A (2009) Camouflage, communication and thermoregulation: lessons from colour changing organisms. Philos Trans R Soc B Biol Sci 364:463-470. doi: 10.1098/rstb.2008.0254

Sulieman Y, Pengsakul T (2015) Non-hemoparasitic protozoa of the subdesert toad, Amietophrynus (Bufo) xeros (Anura: Bufonidae). Int J Research- Granthaalayah 2:89-92

Taffs LF (1976) Pinworm infections in laboratory rodents: a review. Lab Anim 10:113. doi: $10.1258 / 002367776780948862$

Taft SJ, Rosenfield RN, Bielefeldt J (1994) Avian hematozoa of adult and nestling cooper's hawks (Accipiter cooperi) in Wisconsin. J Helminthol Soc Washingt $61: 146-148$

Tinsley RC (1989) The effects of host sex on transmission success. Paras. Today 5,6:190-195

Thayer G (1909) Concealing-coloration in the animal kingdom: an exposition of the laws of disguise through color and pattern: being a summary of Abbott $\mathrm{H}$. Thayer's Discoveries. MacMillan, New York

Théron A, Rognon A, Pagès JR (1998) Host choice by larval parasites: a study of Biomphalaria glabrata snails and Schistosoma mansoni miracidia related to host size. Parasitol Res 84:727-732. doi: 10.1007/s004360050477

Thieltges DW, Amundsen PA, Hechinger RF, et al. (2013) Parasites as prey in aquatic food webs: implications for predator infection and parasite transmission. Oikos 122:1473-1482. doi: 10.1111/j.1600-0706.2013.00243.x

Thompson J, Pellmyr O (1991) Evolution of oviposition behavior and host preference in Lepidoptera. Annu Rev Entomol 36:65 - 89

Tobi DR, Grehan JR, Parker BL (1993) Review of the ecological and economic significance of forest Hepialidae (Insecta: Lepidoptera). For Ecol Manage 56:112. doi: 10.1016/0378-1127(93)90099-9 
Trainer DO, Schildt CS, Hunt RA, Jahn LR (1962) Prevalence of Leucocytozoon simondi among some Wisconsin waterfowl. J Wildl Manage 26:137-143.

Travi BL, Osorio Y, Melby PC, et al. (2002) Gender is a major determinant of the clinical evolution and immune response in hamsters infected with Leishmania spp. Infect Immun 70:2288-2296. doi: 10.1128/IAl.70.5.2288-2296.2002

Tregenza T (1995) Building on the ideal free distribution. Adv Ecol Res 26:253-302

Trillmich F, Wolf JBW (2008) Parent-offspring and sibling conflict in Galapagos fur seals and sea lions. Behav Ecol Sociobiol 62:363-375. doi: 10.1007/s00265007-0423-I

Troscianko J, Lown AE, Hughes AE, Stevens M (2013) Defeating crypsis: detection and learning of camouflage strategies. PLoS One 8:e73733. doi: 10.1371/journal.pone.0073733

Tscharntke T (1992) Cascade effects among four trophic levels: bird predation on galls affects density-dependent parasitism. Ecology 73:1689-1698

Tschirren B, Bischoff LL, Saladin V, Richner H (2007) Host condition and host immunity affect parasite fitness in a bird ectoparasite system. Funct Ecol 21:372-378. doi: 10.1111/j.1365-2435.2007.01235.x

Tseng M, Myers JH (2014) The relationship between parasite fitness and host condition in an insect - virus system. PLoS One 9:e106401. doi: 10.1371/journal.pone.0106401

Tsuji K, Sota T (2010) Sexual differences in flower defense and correlated malebiased florivory in a plant-florivore system. Oikos 119:1848-1853. doi: 10.1111/j.1600-0706.2010.18585.x

Tsuji K, Sota T (2010) Florivores on the dioecious shrub Eurya japonica and the preferences and performances of two polyphagous geometrid moths on male and female plants. Ent Sci 16,3:291-297

Tyler J, Gilliam J (1995) Ideal free distributions of stream fish: a model and test with minnows, Rhinicthys atratulus. Ecology 76:580-592 
Uribe-Mú CA, Quesada M (2006) Preferences, patterns and consequences of branch removal on the dioecious tropical tree Spondias purpurea (Anacardiaceae) by the insect borer Oncideres albomarginata chamela (Cerambycidae). Oikos 112:691-697. doi: 10.1111/j.0030-1299.2006.13957.x

Vaclav R, Prokop P, Fekiac V (2007) Expression of breeding coloration in European green lizards (Lacerta viridis): variation with morphology and tick infestation. Can J Zool Can Zool 85:1199-1206. doi: 10.1139/Z07-102

Verdú M, García-Fayos P, Gleiser G (2004) Mites attack males of the sexually polymorphic tree Acer opalus more harmfully and more often. Funct Ecol 18:592-597. doi: 10.1111/j.0269-8463.2004.00884.x

Verges A, Paul NA, Steinberg PD (2008) Sex and life-history stage alter herbivore responses to a chemically defended red alga. Ecology 89:1334-1343. doi: 10.1890/07-0248.1

Walker W, Roberts CW, Ferguson DJP, et al. (1997) Innate immunity to Toxoplasma gondii is influenced by gender and is associated with differences in interleukin12 and gamma innate immunity to Toxoplasma gondii is influenced by gender and is associated with differences in interleukin-12 and gamma interfe. Infect Immun 65:1119-1121

Wallace AR (1870) Contributions to the theory of natural selection: a series of essays. Macmillan and Co, London

Wardhaugh CW, Blakely TJ, Greig H, et al. (2006) Vertical stratification in the spatial distribution of the beech scale insect (Ultracoelostoma assimile) in Nothofagus tree canopies in New Zealand. Ecol Entomol 31:185-195. doi: 10.1111/j.03076946.2006.00778.x

Warton D, Duursma R, Falster D, Taskinen S (2012) smatr 3 - an R package for estimation and inference about allometric lines. Methods Ecol Evol 3:257 - 259

Waterman JM, Macklin GF, Enright C (2013) Sex-biased parasitism in Richardson's ground squirrels (Urocitellus richardsonii) depends on the parasite examined. Can J Zool 79:73-79. doi: 10.1139/cjz-2013-0151 
Wedekind C, Jakobsen PJ (1998) Male-biased susceptibility to helminth infection: an experimental test with a copepod. Oikos 81:458-462. doi: 10.2307/3546767

Williams A, Antonovics J, Rolff J (2011) Dioecy, hermaphrodites and pathogen load in plants. Oikos 120:657-660. doi: 10.1111/j.1600-0706.2011.19287.x

Williams AC, Flaherty SE, Flaxman SM (2013) Quantitative tests of multitrophic ideal free distribution theory. Anim Behav 86:577-586. doi:

10.1016/j.anbehav.2013.06.013

Wolfe LM (1997) Differential flower herbivory and gall formation on males and females of Neea psychotrioides, a dioecious tree. Source: Biotropica 29:169174. doi: 10.1111/j.1744-7429.1997.tb00021.x

Yoder B, Ryan M, Waring R et al. (1994) Evidence of reduced photosynthetic rates in old trees. Fors Sci 40:513-527

Yule KJ, Burns KC (2014) Drivers of aggregation in a novel arboreal parasite: the influence of host size and infra-populations. Int J Parasitol 45:197-202. doi: 10.1016/j.ijpara.2014.10.007

Zahn A, Rupp D (2004) Ectoparasite load in European vespertilionid bats. J Zool 262:383-391. doi: 10.1017/s0952836903004722

Zhang L-BL, Parsons S, Daszak P, et al. (2010) Variation in the abundance of ectoparasitic mites of flat-headed bats. J Mammal 91:136-143. doi: 10.1644/08-MAMM-A-306R2.1.Key

Zhang Z, Chen L, Saito S, et al. (2000) Possible modulation by male sex hormone of Th1/Th2 function in protection against Plasmodium chabaudi chabaudi AS infection in mice. Exp Parasitol 96:121-129. doi: 10.1006/expr.2000.4572

Zuk M, McKean K a. (1996) Sex differences in parasite infections: patterns and processes. Int J Parasitol 26:1009-1024. doi: 10.1016/S0020-7519(96)00086-0

Zuk M, Stoehr AM (2002) Immune defense and host life history. Am Nat 160 Suppl:S9-S22. doi: 10.1086/342131 
Zvereva E, Lanta V, Kozlov M (2010) Effects of sap-feeding insect herbivores on growth and reproduction of woody plants: a meta-analysis of experimental studies. Oecologia 163,4: 949-960.

Zwieniecki MA, Melcher PJ, Feild TS, Holbrook NM (2004) A potential role for xylemphloem interactions in the hydraulic architecture of trees: effects of phloem girdling on xylem hydraulic conductance. Tree Physiol 24:911-7 


\section{Appendices}

\section{$\begin{array}{lllllllllll}3.0 & 4.5 & 6.0 & 2.0 & 3.5 & 5.0 & 20 & 40 & 60 & 500 & 1500\end{array}$}
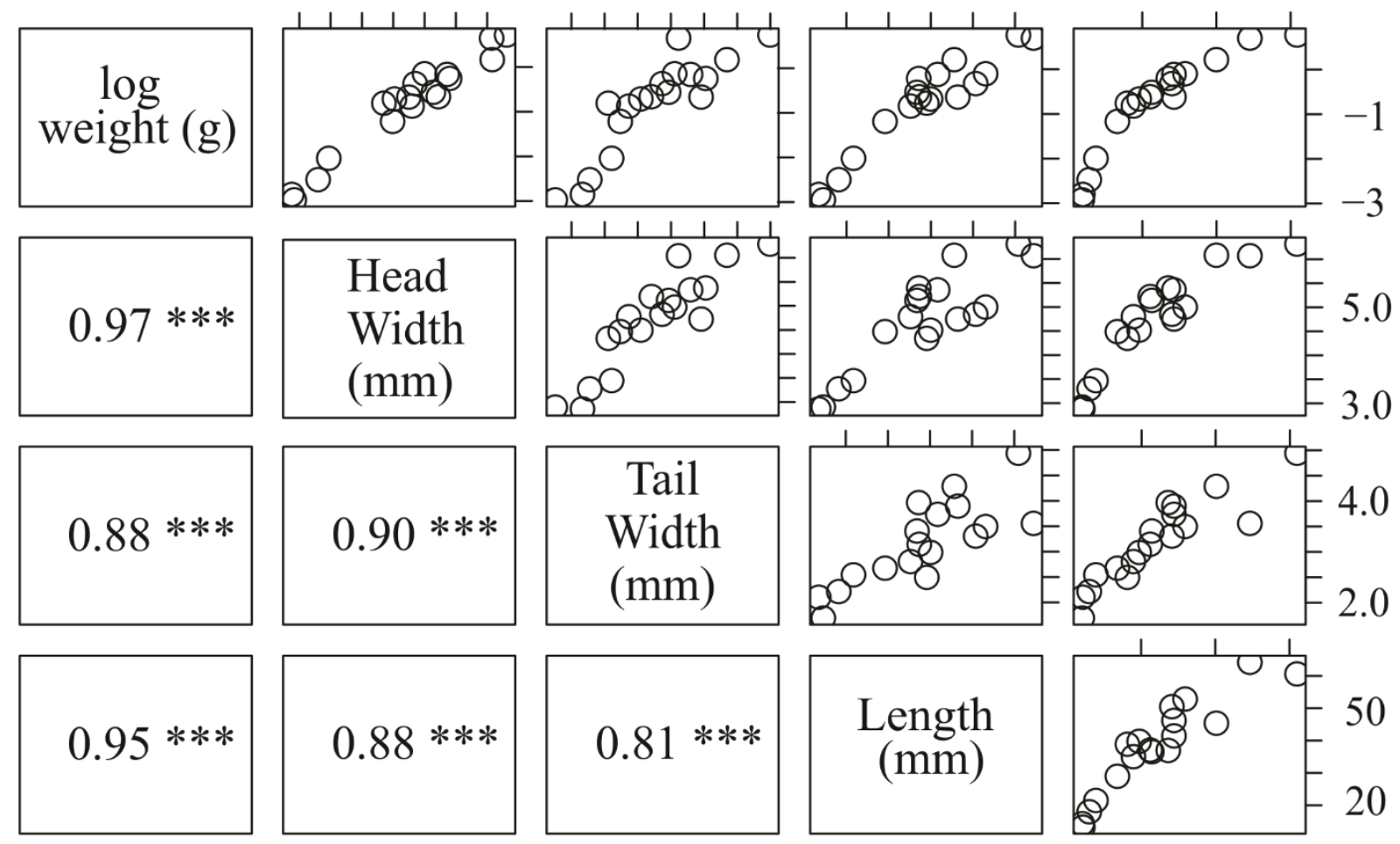

50
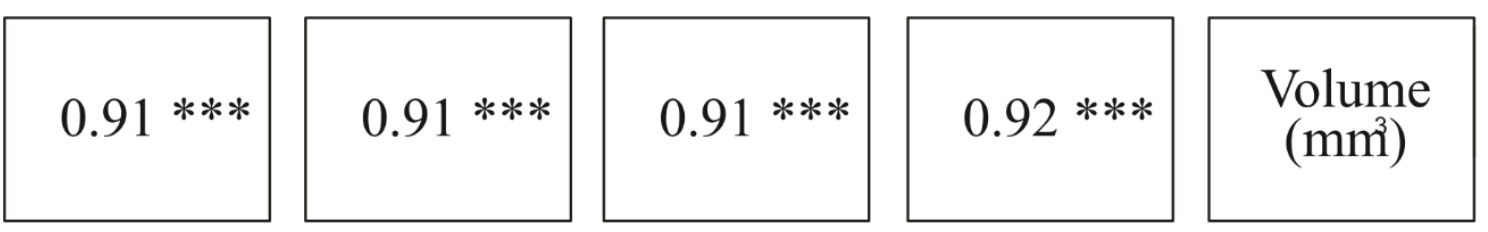

Figure A.1. Correlation matrix of Aenetus virescens body components - weight, head width, tail width, length and volume - used to deduce the allometric relationships between larval body parts. $r^{2}$ values are shown in corresponding boxes in the bottom left of the figure. ${ }^{* *}$ Statistically significant $(P<0.001)$. 

Table A.1. Fifty two plant host - parasite pairings used in meta-analysis in chapter 5. Column one lists host plant order, family, and genus \& species in alphabetical order. Parasites are listed to order, species names where known. Bias in susceptibility is either male $(\mathrm{M})$ or female $(\mathrm{F})$, whether parasite prevalence $(\mathrm{P})$ or intensity (I) was reported by the authors. Sample size refers to the number of hosts inspected, reported where known; any sample sizes that occur in the male column only relates to both male and female sample sizes combined where authors did not specific by gender specifically.

\begin{tabular}{|c|c|c|c|c|c|c|c|}
\hline \multirow[t]{2}{*}{ Host } & \multirow[t]{2}{*}{$\begin{array}{l}\text { Parasite } \\
\text { order }\end{array}$} & \multirow[t]{2}{*}{ Parasite Species } & \multirow[t]{2}{*}{ Bias } & \multirow[t]{2}{*}{$\begin{array}{l}\mathrm{P} \\
\text { or } \\
\mathrm{I}\end{array}$} & $\begin{array}{c}\text { Sample } \\
\text { size }\end{array}$ & \multirow[t]{2}{*}{ Reason for pattern? } & \multirow[t]{2}{*}{ Reference } \\
\hline & & & & & $M$ & & \\
\hline
\end{tabular}

\section{ALISMATALES}

\section{Araceae}

Arisaema triphyllum

Diptera

Unamed sp.

Thysanoptera

Heterothrips arisaemae

Lepidoptera

Unamed sp.

Collembolans

Unamed sp.

\section{AQUIFOLIALES}

Aquifoliaceae

Ilex aquifolium

Diptera

\section{ARECALES}

Arecaceae

F

M

M

M

M

35
33 Reproductive function i.e. pollen availability in plants
not defence or nutrient

\section{Feller et al. (2002)}

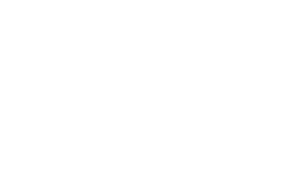

M

P 66
Leaf thickness, sugar
levels, amino-acid
composition Bañuelos \& Kollmann
(2011) 
Chamaedorea

ernesti-augusti

Chamaedorea

pinnatifrons

Chamaedorea

alternans

\section{ASTERALES}

\section{Asteraceae}

B. halimifolia

\section{CARYOPHYLLALES}

\section{Caryophyllaceae}

Silene dioica

\section{Chenopodiaceae}

Atriplex vesicaria

Atriplex canescens

Artiodactyla

Sheep

Artiodactyla

Cattle

\section{Nyctaginaceae}

Neea psychotrioides

Lepidoptera

unamed sp.

Diptera

Cecidomydae sp.

M

\section{Polygonaceae}

Rumex acetosella
Coleoptera
M

Calypthocephala

marginipennis

\section{Calypthocephala}

marginipennis

Trirhabda baeharidis

M

M

45

45 Reproduction-growth trade

off, so lower biomass hence

Cepeda-Cornejo \&

lower herbivory

45 Reproduction-growth trade

off, so lower biomass hence

lower herbivory

$\begin{array}{lll}M & P & 45\end{array}$

45

Reproduction-growth trade

off, so lower biomass hence

lower herbivory

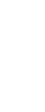


Rumex acetosa

Gastropoda

Arianta arbustorum

M

\section{EPHEDRALES}

Ephedraceae

Ephedra distachya

Ephedra trifurea

Diptera

Diptera

Diptera

\section{ERICALES}

Pentaphylacaceae

Eurya japonica

Lepidoptera

Chloroclystis excisa

M

M

hedromyia

ustjurtensis

Lasioptera ephedrae

L. ephedrieola

Lepidoptera

Alcis angulifera

FAGALES

Myricaceae

Myrica gale

\section{LAMIALES}

\section{Buddlejaceae}

Buddleja cordata

\section{MALPIGHIALES}

\section{Salicaceae}

Populus tremula
Lepidoptera

Acronyctodes

mexicanaria

Lepidoptera unamed sp.

M

F P,I $26 \quad 26$ Higher nutritional quality Unknown

M P 100100 Variation in nutritional quality/nitrogen
L. Ericson (unpubl.

data)

Lucero García-García \& Cano-Santana

T. Elmqvist (unpubl

data)

Patra et al. (2012)

Boecklen (1993)

Tsuji \& Sota (2010)

Tsuji and Sota (2013)

$$
\text { (2015) }
$$$$
\text { (2015) }
$$

Hjältén (1992) 


\begin{tabular}{|c|c|c|c|c|c|c|c|c|}
\hline & & & & & & & $\begin{array}{l}\text { concentration/bark } \\
\text { digestability }\end{array}$ & \\
\hline Salix caprea & Rodentia & Vole sp. & $M$ & & & & & Danell et al. (1991) \\
\hline \multirow[t]{2}{*}{ Salix myrsinifolia } & Rodentia & Vole sp. & $M$ & $\mathrm{P}$ & 100 & 100 & $\begin{array}{l}\text { Nutrient allocation for } \\
\text { flowering/growth }\end{array}$ & Danell et al. (1985) \\
\hline & Rodentia & Vole sp. & $M$ & $P$ & 140 & 140 & $\begin{array}{l}\text { Nutrient allocation for } \\
\text { flowering/growth; (Sig more } \\
\text { males) More resources } \\
\text { stored in male leaves; }\end{array}$ & $\begin{array}{l}\text { Danell et al. (1985), } \\
\text { Elmqvist et al. } \\
\text { (1988),Danell et al. } \\
\text { (1991) }\end{array}$ \\
\hline Salix pentandra & Lagomorpha & Lepus timidus & M & $\mathrm{P}$ & 100 & 100 & $\begin{array}{l}\text { Variation in nutritional } \\
\text { quality/nitrogen } \\
\text { concentration/bark } \\
\text { diaestability }\end{array}$ & Hjältén (1992) \\
\hline Salix viminalis & Diptera & Earis chlorama & $\mathrm{F}$ & $\mathrm{P}$ & 153 & 242 & $\begin{array}{l}\text { Females spend less on } \\
\text { resources }\end{array}$ & Ahman (1997) \\
\hline Salix caprea & Lagomorpha & Lepus timidus & $\mathrm{M}$ & $\mathrm{P}$ & 100 & 100 & $\begin{array}{l}\text { Variation in nutritional } \\
\text { quality/nitrogen } \\
\text { concentration/bark } \\
\text { digestability }\end{array}$ & Hjältén (1992) \\
\hline \multirow[t]{2}{*}{ Salix cinerea } & Insects & unamed sp. & $M$ & $\mathrm{P}$ & 110 & 230 & & Alliende (1989) \\
\hline & Gastropoda & Cepaea nemoralis & M & & & & & \\
\hline Salix elaeagnos & Diptera & Rhabdopaga sp. 5 & $M$ & I & 32 & 32 & shoot length & Kopelke et al. (2003) \\
\hline \multirow[t]{4}{*}{ Salix lasiolepis } & Diptera & Euura lasiolepis & M & 1 & 10 & 11 & Variation in leaf phenology & Boecklen et al. (1990 \\
\hline & Diptera & Nematus sp. & M & & & & & \\
\hline & & & $\mathrm{M}$ & & & & & \\
\hline & Hymenoptera & Phyllocolpa excavata & $M$ & & & & & \\
\hline
\end{tabular}

\title{
NEMALIALES
}

\section{Bonnemaisoniaceae}

\author{
Asparagopsis armata Gastropoda Aplysia parvula
}




\section{PINALES}

\section{Cupressacae}

Juniperus seopulorum Hemiptera Unamed sp.

\section{POALES}

Poaceae

Poa ligularis

$\begin{array}{ll}\text { Artiodactyla } & \text { Sheep } \\ \text { Hemiptera } & \text { Sipha maydis } \\ \text { Insects } & \text { unamed sp. } \\ \text { Rodenta } & \text { unamed sp. }\end{array}$

M

M

M

Rodenta

unamed sp.

\section{Restionaceae}

Hypodiscus aristatus

Hemiptera

Cephalelus sp.

\section{ROSALES}

\section{Elaeagnaceae}

\section{Hippophae}

rhamnoides

\section{Rosaceae}

Rubus chamaemorus

Coleoptera

Unamed sp.

Lepidoptera

Unamed sp.

M

20 moved

M

M P

Reproduction roles

constrain evolution of defense, hance diff in palatability
Graff et al. (2013)

Distan
plants

Augustyn et al. (2013)

L. Ericson (unpubl. data)

Agren (1987)

T. Elmqvist (unpubl.

data) 


\section{SAPINDALES}

Aceraceae

Acer negundo

Acer opalus

Mite

\section{Anacardiaceae}

Spondias purpurea

Lepidoptera

Coleoptera
Unamed sp.

Aceria macrorhynchus

Rothschildia cinta

Oncideres

albomarginata chamela
M

22

allocation to

reproduction and

defense/diff age leaves

sampled

Diff in plant tissue

quality/leaf

thoughness/female

competition for resources

for fruits

$\begin{array}{llll}F & P & 35 & 35\end{array}$

F $\quad$ P $25 \quad 26 \quad$ Better quality of resources

(non-structural

carbohydrates and

nitrogen)/defense
Jing \& Coley (1990)

Verdú et al. (2004)

Maldonado-López et al. (2014)

Uribe-Mú \& Quesada

(2006b) 
Table A.2. Animal host - parasite pairings used in meta-analysis in chapter 5. Column one lists host animal order, family, genus and species in alphabetical order. Parasites are listed to order, species names where known. Bias in susceptibility is either male $(\mathrm{M})$ or female $(\mathrm{F})$, whether parasite prevalence $(\mathrm{P})$ or intensity $(\mathrm{I})$ was reported by the authors. Sample size refers to the number of hosts inspected, reported where known; any sample sizes that occur in the male column only relates to both male and female sample sizes combined where authors did not specific by gender specifically.

\begin{tabular}{|c|c|c|c|c|c|c|c|c|}
\hline Animal Species & Parasite Order & Parasites & Bias & $\begin{array}{l}\mathrm{P} \\
\text { or } \\
\mathrm{I}\end{array}$ & $\begin{array}{c}\text { Sample } \\
\text { size } \\
\text { Male }\end{array}$ & $\begin{array}{l}\text { sample } \\
\text { size } \\
\text { females }\end{array}$ & Reason for pattern & Reference \\
\hline
\end{tabular}

\section{Actinopterygil}

\section{Gasterosteiformes}

\section{Gasterosteidae}

Gasterosteus aculeatus

\begin{tabular}{|c|c|c|c|c|}
\hline Pansporoblastina & Glugea anomala & M & $P, I$ & 343 \\
\hline Cestoda & $\begin{array}{l}\text { Schistocephalus } \\
\text { solidus }\end{array}$ & M & $P, I$ & 327 \\
\hline Nematoda & Nematode sp. & $\mathrm{F}$ & $\mathrm{P}$ & 19762 \\
\hline Cestoda & $\begin{array}{l}\text { Schistocephalus } \\
\text { solidus }\end{array}$ & $\mathrm{F}$ & $P$ & 19763 \\
\hline Trematoda & Bunodera sp. & M & $\mathrm{P}$ & 19760 \\
\hline Cestoda & $\begin{array}{l}\text { Cyathocephalus } \\
\text { truncatus }\end{array}$ & $M$ & $P$ & 19761 \\
\hline
\end{tabular}

\section{2}

Sexual selection, different habitats,

Behaviour

680

Immune system

different habitats, dietary differences, infected females could have higher mortality therefore were not sampled?

ecological aspects dietary differences

Reimchen \& Nosil (2001) 


\section{Perciformes}

Centrarchidae

Lepomis gibbosus

Monogenea

Onchocleidus dispar

$M$

Salmoniformes

\section{Salmonidae}

Salmo trutta

Trematoda

Trematoda

Amphibia

Anura

Ranidae

Rana temporaria

Trematoda
Trematoda

Nematoda

Trematoda

Scaphiopus couchii

Arthroleptidae

Leptopelis hyloides

\section{Trematoda}

Rhizophydiales
Gorgoderina

vitelliloba

Polystoma

integerrimum

Rhabdias bufonis

Pseudodiplorchis

americanus

Batrachochytrium
Discocotyle sagittata

M

Crepidostomum

farionis
Ecological factors

Hockley et

al.( 2011)

Paling

(1965)

Thomas

(1964)

Lee \& Bass

(1960)

M

M

M

dendrobatidis
M

21
9

Breeding behaviour

means makes

congregate for

longer ininfected

sites.
Imasuen et al. 


\section{Bufonidae}

Amietophrynus xeros

Anaxyrus americanus

Rhinella marina

\section{Dicroglossidae}

\section{Euphlyctis cyanophlyctis}

\begin{tabular}{|c|c|}
\hline Protozoa & Balantidium sp. \\
\hline protozoa & Nyctotherus sp. \\
\hline protozoa & Opalina sp. \\
\hline Opalinida & Protoopalina sp \\
\hline Nematoda & $\begin{array}{l}\text { Cosmocercoides } \\
\text { variabilis }\end{array}$ \\
\hline Pentastomida & Raillietiella frenatus \\
\hline Trematoda & Diplodiscus mehrai \\
\hline Trematoda & Ganeo tigrinus \\
\hline Trematoda & $\begin{array}{l}\text { Gorgonderina } \\
\text { elliptica }\end{array}$ \\
\hline Trematoda & $\begin{array}{l}\text { Indopleurogenes } \\
\text { yamaguti }\end{array}$ \\
\hline Trematoda & $\begin{array}{l}\text { Loxogenes } \\
\text { jammuensis }\end{array}$ \\
\hline Trematoda & Mehraorchis ranarum \\
\hline
\end{tabular}

Behaviour

Sulieman \&

Pengsakul

(2015)

36

36

36

25

encounter rate

Joy \& Bunten (1997)

Kelehear et al. (2012)

Mostly phenotypic

traits; increased

immune response -

heavier spleen and

larger testes
Verma \& Singh (2000) 


\begin{tabular}{|c|c|c|c|c|c|c|c|c|}
\hline \multirow{7}{*}{ Hoplobatrachus tigerinus } & Trematoda & Prostocus himalayai & $\mathrm{F}$ & & & & & \\
\hline & Trematoda & Diplodiscus mehrai & M & & & & & \\
\hline & Trematoda & Ganeo tigrinus & M & & & & & \\
\hline & Trematoda & $\begin{array}{l}\text { Gorgonderina } \\
\text { elliptica }\end{array}$ & M & & & & & \\
\hline & Trematoda & $\begin{array}{l}\text { Indopleurogenes } \\
\text { yamaguti }\end{array}$ & M & & & & & \\
\hline & Trematoda & $\begin{array}{l}\text { Loxogenes } \\
\text { jammuensis }\end{array}$ & M & & & & & \\
\hline & Trematoda & Prostocus himalayai & M & & & & & \\
\hline \multicolumn{9}{|l|}{ Hyperoliidae } \\
\hline Afrixalus dorsalis & Rhizophydiales & $\begin{array}{l}\text { Batrachochytrium } \\
\text { dendrobatidis }\end{array}$ & M & $P$ & 62 & 46 & $\begin{array}{l}\text { Breeding behaviour } \\
\text { means congregate } \\
\text { for longer at infected } \\
\text { sites. }\end{array}$ & $\begin{array}{l}\text { Imasuen et al. } \\
(2011)\end{array}$ \\
\hline Afrixalus nigeriensis & Rhizophydiales & $\begin{array}{l}\text { Batrachochytrium } \\
\text { dendrobatidis }\end{array}$ & M & $P$ & 35 & 7 & & \\
\hline Afrixalus paradorsalis & Rhizophydiales & $\begin{array}{l}\text { Batrachochytrium } \\
\text { dendrobatidis }\end{array}$ & M & $P$ & 5 & 1 & & \\
\hline Hyperolius concolor & Rhizophydiales & $\begin{array}{l}\text { Batrachochytrium } \\
\text { dendrobatidis }\end{array}$ & M & $P$ & 3 & 1 & & \\
\hline Hyperolius fusciventris burtoni & Rhizophydiales & $\begin{array}{l}\text { Batrachochytrium } \\
\text { dendrobatidis }\end{array}$ & M & $P$ & 9 & 6 & & \\
\hline
\end{tabular}




\begin{tabular}{|c|c|c|c|c|c|}
\hline Hyperolius picturatus & Rhizophydiales & $\begin{array}{l}\text { Batrachochytrium } \\
\text { dendrobatidis }\end{array}$ & M & $P$ & 14 \\
\hline Hyperolius sp. 1 & Rhizophydiales & $\begin{array}{l}\text { Batrachochytrium } \\
\text { dendrobatidis }\end{array}$ & M & $P$ & 10 \\
\hline Hyperolius sp. 2 & Rhizophydiales & $\begin{array}{l}\text { Batrachochytrium } \\
\text { dendrobatidis }\end{array}$ & $\mathrm{M}$ & $P$ & 6 \\
\hline Hyperolius sp. 3 & Rhizophydiales & $\begin{array}{l}\text { Batrachochytrium } \\
\text { dendrobatidis }\end{array}$ & M & $P$ & 4 \\
\hline Hyperolius sp. 4 & Rhizophydiales & $\begin{array}{l}\text { Batrachochytrium } \\
\text { dendrobatidis }\end{array}$ & M & $P$ & 3 \\
\hline Hyperolius sylvaticus & Rhizophydiales & $\begin{array}{l}\text { Batrachochytrium } \\
\text { dendrobatidis }\end{array}$ & M & $P$ & 15 \\
\hline \multicolumn{6}{|l|}{ Phrynobatrachidae } \\
\hline Phrynobatrachus calcaratus & Rhizophydiales & $\begin{array}{l}\text { Batrachochytrium } \\
\text { dendrobatidis }\end{array}$ & M & $P$ & 18 \\
\hline Phrynobatrachus liberiensis & Rhizophydiales & $\begin{array}{l}\text { Batrachochytrium } \\
\text { dendrobatidis }\end{array}$ & $M$ & $P$ & 20 \\
\hline
\end{tabular}




\section{Pipidae}

Xenopus tropicalis

Rhizophydiales

Batrachochytrium

M dendrobatidis

\section{Ptychadenidae}

Ptychadena longirostris

Rhizophydiales

Batrachochytrium dendrobatidis

M P 23

4

Ptychadena pumilio

Rhizophydiales

Batrachochytrium dendrobatidis

$\begin{array}{lll}\text { M } & \mathrm{P} & 17\end{array}$

\section{Ranidae}

Amnirana albolabris

Rhizophydiales

Batrachochytrium dendrobatidis

\section{M}

P

Rana pipiens

Nematoda

Rhabdias ranae

$\mathrm{P}, \quad 70$

Nematoda

64

Rana sylvatica
Rhabdias ranae
F $\quad$ I,$\quad 70$
Dare \&

Difference in

selection of thermal

clines; No difference

in juveniles

\section{Rhacophoridae}


Chiromantis rufescens

Rhizophydiales

Batrachochytrium

dendrobatidis

\section{Salamandridae}

Calotriton asper

Nematoda
Trematoda

\section{Arachnida}

\section{Ixodida}

\section{Ixodidae}

Rhipicephalus appendiculatus

Protoza
Protozoa
Protoza

Aves

\section{Accipitriformes}

\section{Accipitridae}

Accepiter cooperii

$\begin{array}{ll}\text { Hematozoa } & \text { Haemoproteus } \mathrm{sp} \\ \text { Protozoa } & \text { Leishmania toddi }\end{array}$

M

F $\quad P, I \quad 73$ terdentatum

Brachycoelium

salamandrae

Theileria parva

F

Theileria lawrencei $\quad \mathrm{M}$

Theileria taurotragi
Irvin et al.

(1981)

Young et al.

(1975)

Young et al. (1980)

Taft et al.

(1994)

\section{Anseriformes}

Anatidae

Anas platyrhynchos

Protozoa

Leishmania simond

M P, 86 


\section{Cathartidae}

Cathartes aura

Diptera Mosquito sp.

M

aviour, unequa

Burkett-

exposure

Cadena et al.

(2014)

\section{Ciconiiformes}

\section{Ciconiidae}

Mycteria americana

Diptera

Mosquito sp.

$\begin{array}{lll}M & P & 24\end{array}$

5

\section{Falconiformes}

Falconidae

Falco naumanni

Falco tinnunculus

$\begin{array}{ll}\text { Hematozoa } & \begin{array}{l}\text { Haemosporidia } \\ \text { tinnunculi } \\ \text { Haemoproteus } \\ \text { brachiatus } \\ \text { Promatomoa }\end{array} \\ \text { Haemoteus } \\ \text { tinnunculi }\end{array}$$$
\text { F }
$$$$
M
$$

P, 168

\section{Reproduction}

raproduction

Tella et al.

(1996)

Korpimaki et al. (1995)

\section{Fringillidae}

\section{Carduelinae}

Linaria cannabina

Astigmata

\section{Galliformes}

Numididae

Numida meleagris

Proctophyllodes

M

\begin{tabular}{|c|c|c|c|c|c|c|c|}
\hline Nematoda & $\begin{array}{l}\text { Gongylonema } \\
\text { congolense }\end{array}$ & $\mathrm{F}$ & $P, I$ & 25 & 23 & $\begin{array}{l}\text { Male behaviour \& } \\
\text { dietary differences }\end{array}$ & $\begin{array}{l}\text { Davies et al. } \\
(2008)\end{array}$ \\
\hline Cestoda & $\begin{array}{l}\text { Hymenolepis } \\
\text { cantaniana }\end{array}$ & $\mathrm{F}$ & $P, I$ & 25 & 23 & & \\
\hline Acanthocephala & $\begin{array}{l}\text { Mediorhynchus } \\
\text { gallinarum }\end{array}$ & $\mathrm{F}$ & $P, I$ & 25 & 23 & & \\
\hline Cestoda & Numidella numida & $\mathrm{F}$ & $P, I$ & 25 & 23 & & \\
\hline
\end{tabular}

Blanco et al.

(1999)

pinnatus

avies et al. 


$\begin{array}{llllll}\text { Cestoda } & \text { Octopetalum numida } & \text { F } & \text { P,I } & 25 & 23 \\ \text { Cestoda } & \text { Raillietina pintneri } & \text { F } & \text { P,I } & 25 & 23 \\ \text { Nematoda } & \text { Subulura dentigera } & \text { F } & \text { P,I } & 25 & 23 \\ \text { Nematoda } & \text { Subulura suctoria } & \text { F } & \text { P,I } & 25 & 23 \\ \text { Nematoda } & \text { Cyrnea eurycerca } & \text { M } & \text { P,I } & 25 & 23 \\ \text { Cestoda } & \text { Retinometra sp. } & \text { M } & \text { P,I } & 25 & 23\end{array}$

\section{Phasianidae}

Bonasa noasia

Nematoda

Ascaridia compar

M

P,I 115

Lyrurus tetrix

Nematoda

Ascaridia compar

M

191

87

Tetrao urogallus

Nematoda

Ascaridia compar

M P

77

51

3

23

23

23

29 SSD - Body size \& Isomursu et growth rates; al. (2006)

juveniles more

infected than adults;

androgen-immunity

only weak

Aramidae 


\section{Passeriformes}

\section{Cardinalidae}

Cardinalis cardinalis

\section{Paridae}

Parus major

Protozoa

Plasmodium sp.

M I

61

Parulidae

Vermivora peregrina

Diptera

Mosquito sp.

M

P

3

2

\section{Pelecaniformes}

\section{Ardeidae}

Ardea alba

Ardea herodias

Diptera

Mosquito sp.

Diptera

Mosquito sp.

Diptera

Mosquito sp.

Diptera

Nyctanassa violacea

Diptera

Nycticorax nycticorax

\section{Suliformes}

\section{Anhingidae}




\section{Phalacrocoracidae}

Phalacrocorax auritus

Nematoda

Contracaecum spp.

M

163

size; possibly sex

biased exposure or

immunocompetence

Trematoda

Drepanocaphalus

$\begin{array}{lll}M & P, I & 163\end{array}$

102

Blattarian

Blattarian

Blattarian

\section{Coleoptera}

Coleopteran

Coleopteran

Coleopteran

Coleopteran

Coleopteran

Coleopteran

\section{Nematoda \\ Acanthocephala}

Nematoda

\section{Acanthocephalan}

Nematode sp.

Nematoda

Nematode sp.

Nematoda

Nematode sp.

Nematoda

Nematoda

Nematoda

Cestoda
F

F

M

I,

F

F

F

M
Dobrovolny and Ackert

(1934)

Lackie (1972)

Ward et al.

(2001)

Fincher et al.

(1969)

Keyme (1982) 
Diptera

Coelopidae

Coelopa frigida

Acarina

Thinoseius fucicola

$\begin{array}{lll}M & P, I & 685\end{array}$

832

host size \&

ecological

Gilburn et al.

differences; phoresy

Coelopa pilipes

Acarina

Thinoseius fucicola

$\begin{array}{lll}M & P, I & 685\end{array}$

832

Dipteran sp.

Nematoda

Nematode sp.

F

Dipteran sp.

Nematoda

Nematode sp.

Welch (1959)

Welch (1959)

\section{Odonata}

Odonate sp.

Mesostigmata

Unamed sp.

Andres and

Cordero

(1998)

\section{Orthoptera}

Gryllidae

Gryllodes sigillatus

Nematoda

Mehdinema alii

M

P

200

Orthopteran sp.

Nematoda

Nematode sp.

M

Orthopteran sp.

Protozoa

Protozoan

Orthopteran sp.

Protozoa

Protozoan

\section{Malacostraca}

\section{Amphipoda}

Gammaridae

Gammarus pulex

Ward et al (1986)

\section{Paraleptamphopidae}


Paraleptamphopus

subterraneus

\section{Decapoda}

\section{Astacoidea}

Crayfish

\section{Varunidae}

Hemigrapsus nudus

Hemigrapsus oregonensis

Mammalia

\section{Artiodactyla}

\section{Bovidae}

Rupicapra rupicapra rupicapra Nematoda

$$
\text { Nematoda }
$$

Ostertagia $\mathrm{sp}$

$\begin{array}{lll}M & P, I & 45\end{array}$

Nematoda

Nematoda

Nematoda

Nematoda
Paragonimus

kellicotti

Portunion conformis

Portunion conformis
$\begin{array}{lll}M & P, I & 49\end{array}$

M

$\mathrm{F}$

F

Stromberg et

al. (1978)

Kuris et al.

(1980)

Morand et al.

(2004)
Steroid levels; Hoby et al.

testosterone, cortisol (2006) oestrogen
Not related to SSD;

immunocompetence
53 


$\begin{array}{llllll}\text { Nematoda } & \text { Chabertia sp. } & \text { M } & \text { P,I } & 45 & 53 \\ \text { Nematoda } & \begin{array}{l}\text { Oesophagostomum } \\ \text { sp. }\end{array} & \text { M } & \text { P,I } & 45 & 53 \\ \text { Nematoda } & \text { Trichuris sp. } & \text { M } & \text { P,I } & 45 & 53 \\ \text { Nematoda } & \text { Capillaria sp. } & \text { M } & \text { P,I } & 45 & 53 \\ \text { Cestoda } & \text { Moniezia sp. } & \text { M } & \text { P,I } & 45 & 53 \\ \text { Cestoda } & \text { Toxocara sp. } & \text { M } & \text { P,I } & 45 & 53 \\ \text { Trematoda } & \text { Dicrocoelium sp } & \text { M } & \text { P,I } & 45 & 53 \\ \text { Trematoda } & \text { Neostrongylus sp. } & \text { M } & \text { P,I } & 45 & 53 \\ \text { Nematoda } & \text { Muellerius sp } & \text { M } & \text { P,I } & 45 & 53 \\ & & & & & \\ \text { Nematoda } & \text { Protostrongylus sp. } & \text { M } & \text { P,I } & 45 & 53 \\ & & & & & \\ \text { Nematoda } & \text { Cysocaulus sp. } & \text { M } & \text { P,I } & 45 & 53\end{array}$


Nematoda

Arachnida

Tragelaphus strepsiceros

\section{Cervidae}

Rangifer tarandus tarandus

Diptera

Nematoda

\section{Artiodactyla}

Suidae

Sus scrofa

\section{Carnivora}

Canidae

Canis familiaris

Nematoda

Felidae

Felis catus

Mustelidae

Cestoda

Nematoda

Nematoda

Nematoda

Nematoda
Dictyocaulus sp.

Amblyomma

hebraeum

Hypoderma tarandi

Elaphostrongylus

rangiferi

\section{T. solium}

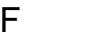

48

Metastrongylus apri

Metastrongylus

pudendotectus

Metastrongylus salmi

$\begin{array}{lll}M & P, I & 10 \\ M & P, I & 10 \\ M & P, I & 10\end{array}$

Dirofilaria immitis

M

Dirofilaria immitis

M
Horak et al.

(1987)

Folstad et al. (1989) adults) Adults)

avoidance of parasites ovipositing

Seasonal cycle

Halvorsen et al. (1985)

Morales-

Montor et al. (2002)

\section{androgen}

testosterone and

high female

hormones

Senlik et al. 
Martes americana

Trematoda

Alaria taxideae

M

\section{Procyonidae}

Procyon lotor

\section{Chiroptera}

Molossidae

Tadarida brasiliensis

\section{Mormoopidae}

Mormoops blainvillei

Nematoda

Baylisascaris

procyonis

\section{Arachnida}

Mesostigmata Ewingana inaequalis

Notoedres

lasionycteris

Capillaria pusilla $\begin{array}{llllll}\text { Siphonaptera } & \text { Sternopsylla distincta } & \text { F } & \text { P,I } & 43 & 47\end{array}$

Nematoda

$\begin{array}{llll}M & P, I & 43 & 47\end{array}$

$\begin{array}{llll}M & P, I & 43 & 47\end{array}$

P, 12

chigger sp.

Arachnida

Nematoda nemato ceran
F P

F $P, \quad 12$
Poole et al.

(1983)

Evans (2001)

Muñoz et al. (2003)

\section{Helminth bias \\ increased with body mass but ecto \\ parasites did not. \\ Inverse relationship \\ between helminths \\ and ecto-parasites}

Krichbaum et al. (2009) 


\begin{tabular}{|c|c|c|c|c|c|c|c|}
\hline Mesostigmata & Periglischrus mite & $\mathrm{F}$ & $\mathrm{P}$ & 12 & & & \\
\hline Nematoda & Trichobius & $\mathrm{F}$ & $\mathrm{P}$ & 12 & & & \\
\hline Cestoda & $\begin{array}{l}\text { Vampirolepis } \\
\text { christensoni }\end{array}$ & $\mathrm{F}$ & $P_{1}$ & 12 & & & \\
\hline Diptera & Bat fly sp. & M & $P, I$ & 115 & 101 & $\begin{array}{l}\text { not associated with } \\
\text { SSD; greater } \\
\text { chances of lateral } \\
\text { and vertical } \\
\text { transmission in } \\
\text { females }\end{array}$ & $\begin{array}{l}\text { Patterson et } \\
\text { al. (2008) }\end{array}$ \\
\hline Diptera & Bat fly sp. & $\mathrm{F}$ & $P, I$ & 201 & 218 & & \\
\hline
\end{tabular}


Helminth bias

increased with body

mass but ecto

parasites did not

Inverse relationship

between helminths

and ecto-parasites

Arachnida

Chiro discidae

F

P, 8

Collembola springtail sp.

F $\quad$ P, 8

Siphonaptera

Nycterophilia sp.

P, 8

Mesostigmata

Periglischrus mite

F $\quad P, \quad 8$ 


\section{Natalidae}

Natalus tumidirostri

Bat fly sp.

not associated with SSD; greater chances of lateral and vertical

transmission in

females

\section{Noctilionidae}

Noctilio leporinus

Diptera

Bat fly sp.

$\begin{array}{lll}M & P, I & 47\end{array}$

\section{Phyllostomidae}

Anoura latidens

Diptera

Bat fly sp.

$\begin{array}{lll}M & P, I & 52\end{array}$

Artebeus jamaicansis

Mesostigmata

Pmechimys iheringi

F $P$,

Artibeus planirostri

Diptera

Bat fly sp.

F $\quad \mathrm{P}, \mathrm{I} \quad 704$

907

Chrotopterus auritus

Aracnhida

Argasid ticks, mites

bat flies

Bat fly sp.

F

$\mathrm{P}$

Diptera
Patterson et
Gannon \&

Willig (1995)

Patterson et al. (2008)

Presley \& Willig (2008) 
Leptonycteris curasoae

Micronycteris minuta

Monophyllus redmani

Myptis blythii
Diptera

Bat fly sp.

Bat fly sp.

Diptera

Siphonaptera

Nycterophilia sp.

F $\quad P, I \quad 31$

36

Periglischrus mite

F

4

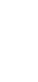

Krichbaum et

increased with body al. (2009)

mass but ecto

parasites did not.

Inverse relationship

between helminths

and ecto-parasites
Christe et al.

(2007) 
Phyllostomus discolor

Phyllostomus elongatus

Diptera

Bat fly sp.

F P I

P,I 60

60

Stenoderma rufum

Sturnira lilium

Mesostigmata

Pmechimys ihering

Diptera

Bat fly sp.

$\begin{array}{lll}M & P, I & 767\end{array}$

1008

\section{Vespertilionidae}

Myotis daubentoni

Myotis lucifugus

Myotis myotis

\begin{tabular}{|c|c|}
\hline Mesostigmata & S. andegavinus \\
\hline Mesostigmata & $\begin{array}{l}\text { Spinturnix } \\
\text { andeaavinus }\end{array}$ \\
\hline Siphonaptera & Myodopsylla insignis \\
\hline Mesostigmata & $\begin{array}{l}\text { Spinturnix } \\
\text { americanus }\end{array}$ \\
\hline
\end{tabular}

Mesostigmata

Mesostigmata

Siphonaptera

Spinturnix myoti
Macronyssidae
Nycteribiidae

$\begin{array}{lll}F & P, I & 10\end{array}$

F $\quad \mathrm{P}, \quad 65$

$\begin{array}{lll} & \text { I } & \\ P, I & 689\end{array}$

$\begin{array}{lll}F & P, I & 689\end{array}$

F P,I 10

10

F $\quad P, \quad 89$

155

F $P, \quad 89$

155 not associated with SSD; greater chances of lateral and vertical

transmission in

females

Patterson et

al. (2008)

chances of lateral

and vertical

transmission in

females

Gannon \&

Willig (1995)

Patterson et

al. (2008)

Christe et al.

(2007)

Lučan (2006)

Host behaviour and

Dick et al.

(2003)

sites

Christe et al.

(2007)

Zahn \& Rupp

(2004) 


\begin{tabular}{|c|c|c|c|c|c|c|c|c|}
\hline & Mesostigmata & Spinturnicidae & $\mathrm{F}$ & $\mathrm{P}$ & 89 & 155 & & \\
\hline & Mesostigmata & Spinturnicidae & $\mathrm{F}$ & $\mathrm{P}$ & 89 & 155 & & \\
\hline \multirow[t]{7}{*}{ Nyctalus leisleri } & Siphonaptera & $\begin{array}{l}\text { Ischnopsllidae } \\
\text { variabilis }\end{array}$ & $\mathrm{F}$ & $P, I$ & 15 & 157 & $\begin{array}{l}\text { time in roost hogher } \\
\text { in females }\end{array}$ & $\begin{array}{l}\text { Kaňuch et al. } \\
\text { (2005) }\end{array}$ \\
\hline & Trematoda & $\begin{array}{l}\text { Ischnopsyllus } \\
\text { intermedius, }\end{array}$ & $\mathrm{F}$ & $P, I$ & 15 & 157 & & \\
\hline & Mesostigmata & Macronyssus flavus & $\mathrm{F}$ & $P, I$ & 15 & 157 & & \\
\hline & Diptera & Nycteribia vexata & $\mathrm{F}$ & $P, I$ & 15 & 157 & & \\
\hline & Diptera & Nycteribia latreillii, & $\mathrm{F}$ & $P, I$ & 15 & 157 & & \\
\hline & Mesostigmata & Spinturnix helvetiae & $F$ & $P, I$ & 15 & 157 & & \\
\hline & Mesostigmata & $\begin{array}{l}\text { Steatonyssus } \\
\text { spinosus }\end{array}$ & $\mathrm{F}$ & $P, I$ & 15 & 157 & & \\
\hline Nyctalus noctula & Mesostigmata & S. acuminata & $\mathrm{F}$ & $P, I$ & 10 & 10 & & $\begin{array}{l}\text { Christe et al. } \\
(2007)\end{array}$ \\
\hline Plecotus auritus & Mesostigmata & S. plecotina & $\mathrm{F}$ & $P, I$ & 10 & 10 & & \\
\hline Tylonycteris pachypus & Mesostigmata & $\begin{array}{l}\text { Macronyssus } \\
\text { radovskyi }\end{array}$ & M & $\mathrm{P}$ & & & $\begin{array}{l}\text { Immune, behaviour, } \\
\text { reproduction }\end{array}$ & $\begin{array}{l}\text { Zhang et al. } \\
\text { (2010) }\end{array}$ \\
\hline Tylonycteris robustula & Mesostigmata & Macronyssus sp. & $M$ & $\mathrm{P}$ & & & & \\
\hline \multicolumn{9}{|l|}{ lipotyphla } \\
\hline \multicolumn{9}{|l|}{ Soricidae } \\
\hline Neomys fodiens & Siphonaptera & Flea sp. & $M$ & $\mathrm{P}, \mathrm{I}$ & 21 & 15 & $\begin{array}{l}\text { not related to SSD, } \\
\text { immunocompetence }\end{array}$ & $\begin{array}{l}\text { Morand et al. } \\
(2004)\end{array}$ \\
\hline Sorex araneus & Siphonaptera & Flea sp. & $M$ & $P, I$ & 124 & 85 & $\begin{array}{l}\text { not related to SSD, } \\
\text { immunocompetence }\end{array}$ & \\
\hline
\end{tabular}


Sorex cinereus

Nematoda

Nematoda

\section{Lagomorpha}

\section{Leporidae}

Oryctolagus cuniculus

Nematoda

\section{Ochotonidae}

Ochotona curzoniae

$$
\begin{aligned}
& \text { Ixodida } \\
& \text { Ixodida }
\end{aligned}
$$

\section{Macroscelidea}

\section{Macroscelididae}

Elephantulus myurus

$$
\text { Liniscus [= Capillaria] }
$$$$
\text { maseri }
$$

M

maseri

Liniscus [= Capillaria] maseri

Trichostrongylus retortaeformis

Hypoderma satyrus

Ixodes crenulatus
Hobbs et al. (1999)

Ci et al. (2008)
102

M

behaviour differences including

Fourie et al. home ranges,

foraging, trail cleaning; small sample size \& overdispersion

\section{Primates}

\section{Hominidae}

Homo sapiens

Protozoa
Nematoda

M

M
Acuna-Soto et al. (2000)

Baboolal \&

Rawlins

(2002) 


$\begin{array}{lll}\text { Nematoda } & \begin{array}{l}\text { Necator americanus } \\ \text { Trematoda }\end{array} & \begin{array}{l}\text { Schistosoma } \\ \text { mansoni }\end{array} \\ \text { Nematoda } & \text { Onchocerca volvulus } & \mathrm{M} \\ \text { Protozoa } & \text { Leishmania donovani } & \mathrm{M} \\ \text { Protozoa } & \begin{array}{l}\text { Leishmania } \\ \text { braziliensis } \\ \text { Wuchereria bancrofti }\end{array} & \mathrm{M} \\ \text { Nematoda } & \begin{array}{l}\text { Plasmodium vivax } \\ \text { protozoa }\end{array} & \mathrm{M} \\ \text { Protozoa } & \begin{array}{l}\text { Plasmodium } \\ \text { falciparum }\end{array} & \mathrm{M} \\ & & \end{array}$

\section{Rodentia}

\section{Cricetidae}

Calomys callosus

Protozoa

Clethrionomys glareolus

Nematoda

Mesocricetus auratus

Nematoda

Protozoa

Protozoa

Trypanosoma cruzi

M I

Capillaria muris

M

Necator americanus

Leishmania

guyanensis

M

M I

95

173

178

M

M

Leishmania

panamensis
Age induced

immunity produces

cytokine secretion

microfilarial densities

increase with worm

burden

Behnke et al.

(1999)

Degu et al.

(2002);

Marguerite et

al. (1999)

Duerr et al.

(2004)

Goble \&

Konopka

(1973)

Jones et al.

(1987)

Kazura et al.

(1984)

Moon \& Cho

(2001)

Weise (1979);

Landgraf

(1994);

Molineaux et

al. (1980)

$\begin{array}{ll}\text { Hormones; } & \text { Do Prado et } \\ \text { oestrogen, interferon } & \text { al. (1998) Do } \\ & \text { Prado et al. } \\ & \text { (1999) } \\ & \text { Lewis \& Twigg } \\ & \text { (1972) } \\ & \text { Jian et al. } \\ & (2003) \\ & \text { Travi et al. } \\ \text { Immune response } & \text { (2002) } \\ & \text { Travi et al. } \\ & (2002)\end{array}$




\begin{tabular}{|c|c|c|c|c|c|c|c|c|}
\hline \multirow[t]{4}{*}{ Microtus arvalis } & Siphonaptera & Flea sp. & $M$ & I & & & Host size & Kiffner et al. \\
\hline & Siphonaptera & Flea sp. & $M$ & I & & & $\begin{array}{l}\text { Species specific } \\
\text { traits - body size }\end{array}$ & $\begin{array}{l}\text { Kiffner et al. } \\
\text { (2014) }\end{array}$ \\
\hline & Siphonaptera & Flea sp. & M & $P, I$ & 290 & 342 & $\begin{array}{l}\text { not related to SSD, } \\
\text { immunocompetence }\end{array}$ & $\begin{array}{l}\text { Morand et al. } \\
(2004)\end{array}$ \\
\hline & Nematoda & Trichuris arvicolae & $\mathrm{F}$ & $P, I$ & 222 & 313 & $\begin{array}{l}\text { females drive } \\
\text { parasite dynamics }\end{array}$ & $\begin{array}{l}\text { (Sanchez etal } \\
\text { (2011) }\end{array}$ \\
\hline \multirow[t]{6}{*}{ Microtus californieus } & Insecta & $\begin{array}{l}\text { Atyphloceras } \\
\text { multidentatus } \\
\text { multidentatus }\end{array}$ & $M$ & & & & & $\begin{array}{l}\text { Stark and } \\
\text { Miles (1962) }\end{array}$ \\
\hline & Insecta & Catallagia wymani & M & & & & & \\
\hline & Siphonaptera & $\begin{array}{l}\text { Hystrichopsylla } \\
\text { linsdalei }\end{array}$ & M & & & & & \\
\hline & Siphonaptera & Malaraeus telchinus & M & & & & & \\
\hline & Siphonaptera & Nosopsyllus fasciatus & M & & & & & \\
\hline & Siphonaptera & $\begin{array}{l}\text { Opisodasys keeni } \\
\text { nesiotus }\end{array}$ & M & & & & & \\
\hline Microtus ochrogaster & Nematoda & Trichinella spiralis & M & I & 10 & 10 & $\begin{array}{l}\text { No relationship to } \\
\text { testosterone, estradil } \\
\text { or corticosterone }\end{array}$ & $\begin{array}{l}\text { Klein et al. } \\
\text { (1999) }\end{array}$ \\
\hline Microtus pennsy/vanicus & Nematoda & Trichinella spiralis & M & I & 10 & 10 & & \\
\hline Myodes glareolus & Nematoda & Mastophorus muris & $\mathrm{F}$ & $P, I$ & 922 & 222 & $\begin{array}{l}\text { Increased exposure } \\
\text { in females due to } \\
\text { dietary differences }\end{array}$ & $\begin{array}{l}\text { Grzybek et al. } \\
(2014)\end{array}$ \\
\hline \multirow[t]{2}{*}{ Myodes glareolus } & Siphonaptera & Flea sp. & M & I & & & Host size & $\begin{array}{l}\text { Kiffner et al. } \\
(2013)\end{array}$ \\
\hline & Siphonaptera & Flea sp. & M & I & & & $\begin{array}{l}\text { Species specific } \\
\text { traits - body size }\end{array}$ & $\begin{array}{l}\text { Kiffner et al. } \\
\text { (2014) }\end{array}$ \\
\hline
\end{tabular}




\begin{tabular}{|c|c|c|c|c|c|c|c|c|}
\hline & Siphonaptera & Flea sp. & $\mathrm{M}$ & $P, I$ & 854 & 747 & $\begin{array}{l}\text { not related to SSD, } \\
\text { rather } \\
\text { immunocompetence }\end{array}$ & $\begin{array}{l}\text { Morand et al. } \\
(2004)\end{array}$ \\
\hline \multirow[t]{2}{*}{ Oligoryzomys nigripes } & Phthiraptera & Hoplopleura imparata & M & I & 91 & & sex and locality & $\begin{array}{l}\text { (Fernandes e } \\
\text { al. (2012) }\end{array}$ \\
\hline & Phthiraptera & $\begin{array}{l}\text { Hoplopleura } \\
\text { travassosi }\end{array}$ & $\mathrm{M}$ & I & 91 & & sex and locality & \\
\hline \multirow[t]{6}{*}{ Peromyscus maniculatus } & Insecta & $\begin{array}{l}\text { Atyphloceras } \\
\text { multidentatus } \\
\text { multidentatus }\end{array}$ & M & & & & & $\begin{array}{l}\text { Stark and } \\
\text { Miles (1962) }\end{array}$ \\
\hline & Insecta & Catallagia wymani & M & & & & & \\
\hline & Siphonaptera & $\begin{array}{l}\text { Hystrichopsylla } \\
\text { linsdalei }\end{array}$ & $\mathrm{M}$ & & & & & \\
\hline & Siphonaptera & Malaraeus telchinus & $\mathrm{M}$ & & & & & \\
\hline & Siphonaptera & Nosopsyllus fasciatus & $\mathrm{M}$ & & & & & \\
\hline & Siphonaptera & $\begin{array}{l}\text { Opisodasys keeni } \\
\text { nesiotus }\end{array}$ & M & & & & & \\
\hline \multirow[t]{6}{*}{ Reithrodontotnys megalotis } & Insecta & $\begin{array}{l}\text { Atyphloceras } \\
\text { multidentatus } \\
\text { multidentatus }\end{array}$ & M & & & & & \\
\hline & Insecta & Catallagia wymani & $\mathrm{M}$ & & & & & \\
\hline & Siphonaptera & $\begin{array}{l}\text { Hystrichopsylla } \\
\text { linsdalei }\end{array}$ & $\mathrm{M}$ & & & & & \\
\hline & Siphonaptera & Malaraeus telchinus & $\mathrm{M}$ & & & & & \\
\hline & Siphonaptera & Nosopsyllus fasciatus & M & & & & & \\
\hline & Siphonaptera & $\begin{array}{l}\text { Opisodasys keeni } \\
\text { nesiotus }\end{array}$ & $\mathrm{M}$ & & & & & \\
\hline \multirow[t]{3}{*}{ Scapteromys aquaticus } & Arachnida & $\begin{array}{l}\text { Eutrombicula } \\
\text { alfreddugesi }\end{array}$ & $\mathrm{F}$ & $P, I$ & 33 & 31 & & \multirow[t]{3}{*}{$\begin{array}{l}\text { Lareschi } \\
(2006)\end{array}$} \\
\hline & Arachnida & $\begin{array}{l}\text { Gigantolaelaps } \\
\text { wolffshoni }\end{array}$ & $\mathrm{F}$ & $P, I$ & 33 & 31 & & \\
\hline & Mesostigmata & Ornithonyssus bacoti & $\mathrm{F}$ & $P, I$ & 33 & 31 & & \\
\hline
\end{tabular}




\begin{tabular}{|c|c|c|c|c|c|c|c|}
\hline Siphonaptera & $\begin{array}{l}\text { Polygenis (Polygenis) } \\
\text { axius axius }\end{array}$ & $F$ & $P, I$ & 33 & 31 & & \\
\hline Arachnida & $\begin{array}{l}\text { Androlaelaps } \\
\text { fahrenholzi }\end{array}$ & M & $P, I$ & 33 & 31 & & \\
\hline Arachnida & $\begin{array}{l}\text { Androlaelaps } \\
\text { rotundus }\end{array}$ & M & $\mathrm{P}, \mathrm{I}$ & 33 & 31 & & \\
\hline Phthiraptera & $\begin{array}{l}\text { Hoplopleura } \\
\text { scapteromydis }\end{array}$ & M & $P, I$ & 33 & 31 & & \\
\hline Ixodida & Laelaps echidninus & M & $P, I$ & 33 & 31 & & \\
\hline Mesostigmata & Laelaps manguinhosi & M & $P, I$ & 33 & 31 & & \\
\hline Mesostigmata & $\begin{array}{l}\text { Laelaps } \\
\text { paulistanensis }\end{array}$ & M & $P, I$ & 33 & 31 & & \\
\hline Siphonaptera & $\begin{array}{l}\text { Polygenis } \\
\text { (Neopolygenis) } \\
\text { atopus }\end{array}$ & M & $P, I$ & 33 & 31 & & \\
\hline Siphonaptera & $\begin{array}{l}\text { Polygenis } \\
\text { (Neopolygenis) } \\
\text { massoiai }\end{array}$ & M & $P, I$ & 33 & 31 & & \\
\hline Siphonaptera & $\begin{array}{l}\text { Polygenis (Polygenis) } \\
\text { bohlsi bohlsi }\end{array}$ & M & $P, I$ & 33 & 31 & & \\
\hline Siphonaptera & $\begin{array}{l}\text { Polygenis (Polygenis) } \\
\text { platensis platensis }\end{array}$ & M & $P, I$ & 33 & 31 & & \\
\hline Nematoda & Trichuris pampeana & $F$ & $P, I$ & 19 & 26 & ecology & $\begin{array}{l}\text { Rossin et al. } \\
(2010)\end{array}$ \\
\hline Nematoda & Heligmostrongylus sp & M & $\mathrm{P}, \mathrm{I}$ & 60 & 44 & $\begin{array}{l}\text { ecological and } \\
\text { physiological causes }\end{array}$ & $\begin{array}{l}\text { Rossin \& } \\
\text { Malizia (2002) }\end{array}$ \\
\hline Nematoda & Trichuris sp. & M & $P, I$ & 60 & 44 & & \\
\hline Nematoda & $\begin{array}{l}\text { Strongyloides } \\
\text { myopotami }\end{array}$ & $\mathrm{F}$ & $P, I$ & 39 & 42 & ecology & $\begin{array}{l}\text { Rossin et al. } \\
(2010)\end{array}$ \\
\hline Nematoda & Trichuris pampeana & $\mathrm{F}$ & $P, I$ & 39 & 42 & & \\
\hline
\end{tabular}

\section{Muridae}




\begin{tabular}{|c|c|c|c|c|c|c|c|c|}
\hline Acomys cahirinus & Siphonaptera & Flea sp. & $M$ & $P, I$ & 88 & 61 & $\begin{array}{l}\text { Bias strongest in } \\
\text { winter during } \\
\text { reproduction \& } \\
\text { hormone biased }\end{array}$ & $\begin{array}{l}\text { Krasnov et al. } \\
\text { (2005a) }\end{array}$ \\
\hline Acomys russatu & Siphonaptera & Flea sp. & $\mathrm{F}$ & $P, I$ & 43 & 32 & & \\
\hline Aethomys namaquensis & Ixodida & $\begin{array}{l}\text { Haemaphysalis } \\
\text { spinulosa }\end{array}$ & M & I & 256 & & $\begin{array}{l}\text { behaviour } \\
\text { differences including } \\
\text { home ranges, } \\
\text { foraging, trail } \\
\text { cleaning; small } \\
\text { sample size \& } \\
\text { overdispersion }\end{array}$ & $\begin{array}{l}\text { Fourie et al. } \\
\text { (1992) }\end{array}$ \\
\hline \multirow[t]{3}{*}{ Apodemus agrarius } & Siphonaptera & Flea sp. & M & 1 & & & Host size & $\begin{array}{l}\text { Kiffner et al. } \\
(2013)\end{array}$ \\
\hline & Siphonaptera & Flea sp. & M & 1 & & & $\begin{array}{l}\text { Species specific } \\
\text { traits - body sie }\end{array}$ & $\begin{array}{l}\text { Kiffner et al. } \\
(2014)\end{array}$ \\
\hline & Siphonaptera & Flea sp. & M & $P, I$ & 1355 & 1124 & $\begin{array}{l}\text { not related to SSD, } \\
\text { immunocompetence }\end{array}$ & $\begin{array}{l}\text { Morand et al. } \\
(2004)\end{array}$ \\
\hline \multirow[t]{3}{*}{ Apodemus flavicollis } & Nematoda & $\begin{array}{l}\text { Heligmosomoides } \\
\text { polygyrus }\end{array}$ & M & 1 & & & $\begin{array}{l}\text { host behaviour and } \\
\text { immunity }\end{array}$ & $\begin{array}{l}\text { Ferrari et al. } \\
(2007)\end{array}$ \\
\hline & Arachnida & Dermacentor spp & M & & & & & \multirow{2}{*}{$\begin{array}{l}\text { Kiffner et al. } \\
(2013) \\
\text { Morand et al. } \\
(2004)\end{array}$} \\
\hline & Siphonaptera & Flea sp. & M & $P, I$ & 1688 & 1830 & $\begin{array}{l}\text { not related to SSD, } \\
\text { immunocompetence }\end{array}$ & \\
\hline \multirow[t]{2}{*}{ Apodemus sylvaticus } & Nematoda & S. stoma & M & $P, I$ & 91 & 43 & $\begin{array}{l}\text { Immune system, } \\
\text { exposure between } \\
\text { competing males }\end{array}$ & $\begin{array}{l}\text { Behnke et al. } \\
\text { (1999) }\end{array}$ \\
\hline & Nematoda & oxyurid nematodes & M & $P, I$ & 74 & & $\begin{array}{l}\text { Body condition; } \\
\text { reproduction, food } \\
\text { limitation }\end{array}$ & $\begin{array}{l}\text { Diaz \& Alonso } \\
\text { (2003) }\end{array}$ \\
\hline
\end{tabular}




\begin{tabular}{|c|c|c|c|c|c|c|c|c|}
\hline & Nematoda & $\begin{array}{l}\text { Nematospiroides } \\
\text { dubius }\end{array}$ & $M$ & & & & & Elton (1931) \\
\hline & Nematoda & Syphacia obvelata & M & & & & & Elton (1931) \\
\hline & Ixodida & Ixodes ricinus & M & I & 289 & & $\begin{array}{l}\text { larger body size } \\
\text { (SSD) resulting in } \\
\text { immunocompromise } \\
\text { in male }\end{array}$ & $\begin{array}{l}\text { Harrison et al. } \\
(2010)\end{array}$ \\
\hline & Ixodida & Ixodes ricinus & $\mathrm{M}$ & 1 & 290 & & & \\
\hline Apodemus uralensis & Siphonaptera & Flea sp. & $M$ & $P, I$ & 518 & 358 & $\begin{array}{l}\text { not related to SSD, } \\
\text { immunocompetence }\end{array}$ & $\begin{array}{l}\text { Morand et al. } \\
(2004)\end{array}$ \\
\hline Dipodillus dasyurus & Siphonaptera & Flea sp. & M & 1 & & & $\begin{array}{l}\text { Species specific } \\
\text { traits - body size }\end{array}$ & $\begin{array}{l}\text { Kiffner et al. } \\
(2014)\end{array}$ \\
\hline Gerbillus. a. allenvyi & Siphonaptera & Flea sp. & $\mathrm{M}$ & $P, I$ & 490 & 71 & $\begin{array}{l}\text { Bias strongest in } \\
\text { winter during } \\
\text { reproduction \& } \\
\text { hormone biased }\end{array}$ & $\begin{array}{l}\text { Krasnov et al. } \\
\text { (2005a) }\end{array}$ \\
\hline Gerbillus andersoni & Siphonaptera & Flea sp. & $M$ & I & & & Host size & $\begin{array}{l}\text { Kiffner et al. } \\
\text { (2013) }\end{array}$ \\
\hline Gerbillus dasyurus & Siphonaptera & Flea sp. & $\mathrm{M}$ & $P, I$ & 898 & 893 & & $\begin{array}{l}\text { Krasnov et al. } \\
\text { (2005a) }\end{array}$ \\
\hline Gerbillus henleyi & Siphonaptera & Flea sp. & $\mathrm{M}$ & $P, I$ & 68 & 166 & & \\
\hline Gerbillus nanus & Siphonaptera & Flea sp. & M & $P, I$ & 57 & 104 & & \\
\hline
\end{tabular}


Lophuromys kilonzoi

Mastomys natalensis

Meriones crassus

Meriones unguiculatus

Millardia meltada

Mus musculus

$\begin{array}{ll}\text { Siphonaptera } & \text { Flea sp. } \\ \text { Siphonaptera } & \text { Flea sp. } \\ & \\ \text { Siphonaptera } & \text { Flea sp. }\end{array}$

Nematoda

Nematoda

Protozoa

Protozoa

Protozoa

Trematoda
Brachylaima cribbi
F I

M I

M P,I 118

195

Brugia malayi

Nippostrongylus

brasiliensis

\section{Babesia microti \\ Leishmania mexicana}

Plasmodium

chabaudi

M I

M $\quad P, I \quad 499(27 \quad 122(71$ adults)

Adults)

I

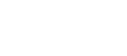

Species specific traits - body size Host size

Bias strongest in winter during reproduction \&

hormone biased

\begin{tabular}{|c|c|}
\hline & el-Bihari \& \\
\hline $\begin{array}{l}\text { immunocompetence } \\
\& \text { avoidance of } \\
\text { parasites ovipositing }\end{array}$ & $\begin{array}{l}\text { Folstad et al. } \\
\text { (1989) }\end{array}$ \\
\hline Immune system & $\begin{array}{l}\text { Aguilar-Delfin } \\
\text { et al. (2001) }\end{array}$ \\
\hline & $\begin{array}{l}\text { Alexander \& } \\
\text { Stimson } \\
\text { (1988) } \\
\text { Benten et al. } \\
\text { (1992); } \\
\text { Benten et al. } \\
\text { (1993); } \\
\text { Benten et al. } \\
\text { (1997); } \\
\text { Wunderlich et } \\
\text { al. (1991); } \\
\text { Zhang et al. } \\
\text { (2000); } \\
\text { Cernetich et } \\
\text { al. submitted }\end{array}$ \\
\hline $\begin{array}{l}\text { Immune system, } \\
\text { female sex }\end{array}$ & $\begin{array}{l}\text { Butcher et al. } \\
\text { (2002) }\end{array}$ \\
\hline
\end{tabular}

Kiffner et al.

Kiffner et al.

(2013)

Krasnov et al. (2005a)

Folstad et al.

Aguilar-Delfin et al. (2001)

Alexander \&

1988)

Benten et al.

1993);

(1997);

al. (1991);

et al.

(2002) 


\begin{tabular}{|c|c|c|c|c|c|c|c|}
\hline Protozoa & Giardia muris & M & 1 & & & Antibody levels & $\begin{array}{l}\text { Daniels \& } \\
\text { Belosevic }\end{array}$ \\
\hline Trematoda & $\begin{array}{l}\text { Schistosoma } \\
\text { mansoni }\end{array}$ & $\mathrm{F}$ & $\mathrm{I}, \mathrm{P}$ & 47 & 20 & Testosterone & $\begin{array}{l}\text { Eloi-Santos et } \\
\text { al. (1992); } \\
\text { Nakazawa et } \\
\text { al. (1997) }\end{array}$ \\
\hline Nematoda & Heterakis spumosa & M & & & & & $\begin{array}{l}\text { Harder et al. } \\
\text { (1992) }\end{array}$ \\
\hline Protozoa & Plasmodium berghei & $\mathrm{M}$ & $\mathrm{P}$ & & & $\begin{array}{l}\text { Testosterone } \\
\text { increase infection } \\
\text { levels }\end{array}$ & $\begin{array}{l}\text { (Kamis \& } \\
\text { Ibrahim } \\
\text { (1989) }\end{array}$ \\
\hline Protozoa & Trypanosoma cruzi & $\mathrm{M}$ & $\mathrm{P}$ & & & Hormone estradril & $\begin{array}{l}\text { Kierszenbaum } \\
\text { (1974); De } \\
\text { Souza et al. } \\
(2001)\end{array}$ \\
\hline Nematoda & Strongyloides ratti & $M$ & I & & & & $\begin{array}{l}\text { Kiyota et al. } \\
\text { (1984) }\end{array}$ \\
\hline Cestoda & Taenia crassiceps & $\mathrm{F}$ & $\mathrm{P}$ & & & $\begin{array}{l}\text { Feminisation caused } \\
\text { by parasitisation; sex } \\
\text { hormones; androgen } \\
\text { constriction }\end{array}$ & $\begin{array}{l}\text { Larralde et al. } \\
\text { (1995); } \\
\text { Morales- } \\
\text { Montor et al. } \\
\text { (2002) }\end{array}$ \\
\hline Protozoa & Leishmania major & $\mathrm{M}$ & 1 & 4 & 5 & testosterone & $\begin{array}{l}\text { Mock \& Nacy( } \\
\text { 1988); } \\
\text { Alexander \& } \\
\text { Stimson } \\
\text { (1988) }\end{array}$ \\
\hline Ixodida & $\begin{array}{l}\text { Haemaphysalis } \\
\text { punctata }\end{array}$ & $\mathrm{F}$ & $P, I$ & 45 & 48 & $\begin{array}{l}\text { host density and } \\
\text { increased male } \\
\text { dispersal }\end{array}$ & $\begin{array}{l}\text { Moravvej et } \\
\text { al. (2016) }\end{array}$ \\
\hline Ixodida & Haemaphysalis sp. & $\mathrm{F}$ & $P, I$ & 45 & 48 & & \\
\hline Arachnida & Ctenophthalmus sp & M & $P, I$ & 45 & 48 & & \\
\hline
\end{tabular}




\begin{tabular}{|c|c|c|c|c|c|c|c|}
\hline Ixodida & Haemolaelaps sp & $\mathrm{M}$ & $P, I$ & 45 & 48 & & \\
\hline Ixodida & Laelaps algericus & M & $P, I$ & 45 & 48 & & \\
\hline Siphonaptera & Nosopsyllus fasciatus & $M$ & $P, I$ & 45 & 48 & & \\
\hline Nematoda & B. pahangi & $M$ & I & & & & $\begin{array}{l}\text { Nakanishi } \\
(1990) ; \\
\text { Nakanishi } \\
(1989)\end{array}$ \\
\hline Cestoda & $\begin{array}{l}\text { Echinococcus } \\
\text { multilocularis }\end{array}$ & $\mathrm{M}$ & $P$ & & & & $\begin{array}{l}\text { Ohbayashi \& } \\
\text { Sakamoto } \\
(1966)\end{array}$ \\
\hline Nematoda & Syphacia obvelata & $\mathrm{M}$ & I & & & & $\begin{array}{l}\text { Okulewicz \& } \\
\text { Perec (2003); } \\
\text { Taffs (1976) }\end{array}$ \\
\hline Nematoda & Brugia malayi & $\mathrm{M}$ & $P$ & & & & $\begin{array}{l}\text { Rajan et al. } \\
(1994)\end{array}$ \\
\hline Nematoda & Muspicea borreli & $\mathrm{M}$ & $\begin{array}{l}\text { I, } \\
\text { P }\end{array}$ & 197 & 168 & Behaviour & $\begin{array}{l}\text { Spratt et al. } \\
(2002)\end{array}$ \\
\hline Insecta & $\begin{array}{l}\text { Atyphloceras } \\
\text { multidentatus } \\
\text { multidentatus }\end{array}$ & $\mathrm{M}$ & $\mathrm{P}$ & & & & $\begin{array}{l}\text { Stark and } \\
\text { Miles (1962) }\end{array}$ \\
\hline Insecta & Catallagia wymani & $\mathrm{M}$ & $\mathrm{P}$ & & & & \\
\hline Siphonaptera & $\begin{array}{l}\text { Hystrichopsylla } \\
\text { linsdalei }\end{array}$ & $\mathrm{M}$ & $P$ & & & & \\
\hline Siphonaptera & Malaraeus telchinus & M & 1 & & & & \\
\hline Siphonaptera & Nosopsyllus fasciatus & $\mathrm{M}$ & I & & & & \\
\hline Siphonaptera & $\begin{array}{l}\text { Opisodasys keeni } \\
\text { nesiotus }\end{array}$ & M & $P$ & & & & \\
\hline
\end{tabular}




\begin{tabular}{|c|c|c|c|c|c|c|c|c|}
\hline & Protozoa & Toxoplasma gondii & $\mathrm{F}$ & 1 & 20 & 20 & Immune & $\begin{array}{l}\text { Walker et al. } \\
\text { (1997); Pung } \\
\text { \& Luster } \\
\text { (1986); } \\
\text { Liesenfeld et } \\
\text { al. (2001); } \\
\text { Roberts et al. } \\
\text { (1995) }\end{array}$ \\
\hline Praomys delectorum & Siphonaptera & Flea sp. & $\mathrm{F}$ & 1 & & & $\begin{array}{l}\text { Species specific } \\
\text { traits - body size }\end{array}$ & $\begin{array}{l}\text { Kiffner et al. } \\
\text { (2014) }\end{array}$ \\
\hline \multirow[t]{10}{*}{ Rattus norvegicus } & Nematoda & $\begin{array}{l}\text { Trichinella } \\
\text { zimbabwensis }\end{array}$ & M & 1 & 25 & \multirow[t]{8}{*}{25} & Immune hormones & \multirow{2}{*}{$\begin{array}{l}\text { Hlaka et al. } \\
(2015) \\
\text { Kamis et al. } \\
(1992)\end{array}$} \\
\hline & Nematoda & $\begin{array}{l}\text { Angiostrongylus } \\
\text { malaysiensis }\end{array}$ & $M$ & I,P & 72 & & $\begin{array}{l}\text { Testosterone } \\
\text { increase infection } \\
\text { levels }\end{array}$ & \\
\hline & Nematoda & Trichinella spiralis & $M$ & $P$ & & & & \multirow{6}{*}{$\begin{array}{l}\text { Mankau \& } \\
\text { Hamilton } \\
\text { (1972) } \\
\text { Rivero et al. } \\
\text { (2002) } \\
\text { Webster \& } \\
\text { Macdonald } \\
\text { (1995) }\end{array}$} \\
\hline & Nematoda & S. venezuelensis & M & 1 & & & & \\
\hline & Mesostigmata & Notoedres muris & $\mathrm{F}$ & 1 & & & & \\
\hline & Nematoda & Capillaria spp. & $\mathrm{M}$ & 1 & & & & \\
\hline & Phthiraptera & Polyplax spinulosa & M & $P$ & & & & \\
\hline & Cestoda & Hymenolepis nana & $\mathrm{F}$ & 1 & & & & \\
\hline & Siphonaptera & $\begin{array}{l}\text { Echidnophaga } \\
\text { gallinacea }\end{array}$ & $M$ & $P, I$ & 124 & 153 & $\begin{array}{l}\text { reproductive } \\
\text { behaviour, home } \\
\text { range, foraging } \\
\text { activity }\end{array}$ & $\begin{array}{l}\text { Soliman et al. } \\
\text { (2001) }\end{array}$ \\
\hline & Mesostigmata & Laelaps nuttalli & $\mathrm{M}$ & $P, I$ & 124 & 153 & & \\
\hline
\end{tabular}




\begin{tabular}{|c|c|c|c|c|c|c|}
\hline Siphonaptera & Leptopsylla segnis & $M$ & $P, I$ & 124 & 153 & \\
\hline Mesostigmata & Ornithonyssus bacoti & $M$ & $P, I$ & 124 & 153 & \\
\hline Phthiraptera & Polyplax spinulosa & $M$ & $P, I$ & 124 & 153 & \\
\hline Mesostigmata & Radfordia ensifera & M & $P, I$ & 124 & 153 & \\
\hline Siphonaptera & Xenopsylla cheopis & $M$ & $P, I$ & 124 & 153 & \\
\hline Insecta & $\begin{array}{l}\text { Atyphloceras } \\
\text { multidentatus } \\
\text { multidentatus }\end{array}$ & $M$ & I & & & $\begin{array}{l}\text { Stark and } \\
\text { Miles (1962) }\end{array}$ \\
\hline Insecta & Catallagia wymani & M & $\mathrm{P}$ & & & \\
\hline Siphonaptera & $\begin{array}{l}\text { Hystrichopsylla } \\
\text { linsdalei }\end{array}$ & M & $\mathrm{I}, \mathrm{P}$ & & & \\
\hline Siphonaptera & Malaraeus telchinus & M & 1 & & & \\
\hline Siphonaptera & Nosopsyllus fasciatus & $M$ & I & & & \\
\hline Siphonaptera & $\begin{array}{l}\text { Opisodasys keeni } \\
\text { nesiotus }\end{array}$ & M & I & & & \\
\hline
\end{tabular}


Rattus rattus

(1)

Rhabdomys pumilio

\begin{tabular}{|c|c|c|c|c|c|c|c|}
\hline Siphonaptera & $\begin{array}{l}\text { Echidnophaga } \\
\text { gallinacea }\end{array}$ & M & $P, I$ & 127 & 115 & $\begin{array}{l}\text { reproductive } \\
\text { behaviour, home } \\
\text { range, foraging } \\
\text { activity }\end{array}$ & $\begin{array}{l}\text { Soliman et al. } \\
\text { (2001) }\end{array}$ \\
\hline Mesostigmata & Laelaps nuttalli & M & $P, I$ & 127 & 115 & & \\
\hline Siphonaptera & Leptopsylla segnis & M & $\mathrm{P}, \mathrm{I}$ & 127 & 115 & & \\
\hline Mesostigmata & Ornithonyssus bacoti & M & $P, I$ & 127 & 115 & & \\
\hline Phthiraptera & Polyplax spinulosa & M & $\mathrm{P}, \mathrm{I}$ & 127 & 115 & & \\
\hline Mesostigmata & Radfordia ensifera & M & $P, I$ & 127 & 115 & & \\
\hline Siphonaptera & Xenopsylla cheopis & M & $P, I$ & 127 & 115 & & \\
\hline Arachnida & $\begin{array}{l}\text { Androlaelaps } \\
\text { dasymys }\end{array}$ & $\mathrm{F}$ & $P, I$ & 217 & 149 & $\begin{array}{l}\text { host realted features } \\
\& \text { biological features } \\
\text { of parasite }\end{array}$ & $\begin{array}{l}\text { Matthee et al. } \\
\text { (2010) }\end{array}$ \\
\hline Insects & Chiastopsylla rossi & $\mathrm{F}$ & $P, I$ & 217 & 149 & & \\
\hline
\end{tabular}




$\begin{array}{llllll}\text { Ixodida } & \text { Hyalomma truncatum } & \text { F } & \text { P,I } & 217 & 149 \\ \text { Mesostigmata } & \text { Laelaps horaki } & \text { F } & \text { P,I } & 217 & 149 \\ \text { Mesostigmata } & \text { Laelaps radovskyi } & \text { F } & \text { P,I } & 217 & 149 \\ \text { Ixodida } & \begin{array}{l}\text { Rhipicephalus } \\ \text { lounsburyi }\end{array} & \text { F } & \text { P,I } & 217 & 149 \\ \text { Arachnida } & \begin{array}{l}\text { Androlaelaps } \\ \text { fahrenholzi }\end{array} & \text { M } & \text { P,I } & 217 & 149 \\ \text { Siphonaptera } & \begin{array}{l}\text { Dinopsyllus ellobius } \\ \text { Siphonaptera }\end{array} & \text { M } & \text { P,I } & 217 & 149 \\ \text { Dinopsyllus tenax } & \text { M } & \text { P,I } & 217 & 149 \\ \text { Ixodida } & \begin{array}{l}\text { Haemaphysalis } \\ \text { aciculifer }\end{array} & \text { M } & \text { P,I } & 217 & 149 \\ \text { Ixodida } & \begin{array}{l}\text { Haemaphysalis } \\ \text { elliptica }\end{array} & \text { M } & \text { P,I } & 217 & 149 \\ \text { Siphonaptera } & \begin{array}{l}\text { Hypsophthalmus } \\ \text { temporis } \\ \text { Ixodida }\end{array} & \text { M } & \text { P,I } & 217 & 149 \\ \text { Ixodes alluaudi } & \text { M } & \text { P,I } & 217 & 149\end{array}$




\begin{tabular}{|c|c|c|c|c|c|c|c|c|}
\hline & Ixodida & Ixodes bakeri & $M$ & $P, I$ & 217 & 149 & & \\
\hline & Ixodida & Ixodes rhabdomysae & $M$ & $P, I$ & 217 & 149 & & \\
\hline & Mesostigmata & Laelaps giganteus & $M$ & $P, I$ & 217 & 149 & & \\
\hline & Siphonaptera & $\begin{array}{l}\text { Listropsylla } \\
\text { agrippinae }\end{array}$ & $M$ & $P, I$ & 217 & 149 & & \\
\hline & Phthiraptera & Polyplax arvicanthis & $\mathrm{M}$ & $P, I$ & 217 & 149 & & \\
\hline & Ixodida & $\begin{array}{l}\text { Rhipicephalus } \\
\text { gertrudae }\end{array}$ & $M$ & $P, I$ & 217 & 149 & & \\
\hline Sekeetamys calurus & Siphonaptera & Flea sp. & $M$ & $P, I$ & 40 & 42 & $\begin{array}{l}\text { Bias strongest in } \\
\text { winter during } \\
\text { reproduction \& } \\
\text { hormone biased }\end{array}$ & $\begin{array}{l}\text { Krasnov et al. } \\
\text { (2005a) }\end{array}$ \\
\hline \multicolumn{9}{|l|}{ Sciuridae } \\
\hline \multirow[t]{3}{*}{ Glaucomys sabrinus } & Arachnida & Haemogamasus reidi & $M$ & I & 10 & 8 & immunocompetence & $\begin{array}{l}\text { Perez-Orella } \\
\text { \& Schulte- } \\
\text { Hostedde } \\
(2005)\end{array}$ \\
\hline & Siphonaptera & $\begin{array}{l}\text { Opisodasys } \\
\text { pseudarctomys }\end{array}$ & $\mathrm{M}$ & 1 & 10 & 8 & & \\
\hline & Siphonaptera & Orchopeas caedens & $M$ & 1 & 10 & 8 & & \\
\hline
\end{tabular}


size in males; males

favour investment in

mating at the

Nematoda

Trichostrongylus retortaeformis

Nematoda

Trichuris sp

M P

38

Nematoda

Trypanoxyuris

(Rodentoxyuris) sciur

$\begin{array}{lll}M & P, I & 38\end{array}$

41

\section{Sciurus vulgaris}

Tamiasciurus hudsonicus

Urocitellus richardsonii
Protozoa

Eimeria sciurorum

Opisodasys

pseudarctomys

\section{Siphonaptera}

Siphonaptera

Orchopeas caedens

Strongyloides sp.

Nematoda

Siphonaptera

Flea sp. expense of immunity

$\begin{array}{lll}M & P, I & 38\end{array}$
Immune system,

Different habitat,

behaviour

48

males have wekaer

immunity; fleas grow

more on males

48

48
Bertolino et al. (2003)

Gorrell \&

Schulte-

Hostedde (2008)

Waterman et al. (2013) 


\begin{tabular}{|c|c|c|c|c|c|c|}
\hline & Nematoda & Nematode sp. & M & $\begin{array}{l}\mathrm{I}, \\
\mathrm{P}\end{array}$ & 32 & 32 \\
\hline \multirow[t]{5}{*}{ Xerus inauris } & Siphonaptera & Flea sp. & $\mathrm{F}$ & I & 44 & 52 \\
\hline & Siphonaptera & Flea sp. & M & I & 44 & 52 \\
\hline & Nematoda & Roundworm sp. & $\mathrm{F}$ & I & 44 & 52 \\
\hline & Nematoda & Hookworms sp. & $\mathrm{F}$ & I & 44 & 52 \\
\hline & Conoidasida & Coccidia sp. & $\mathrm{F}$ & I & 44 & 52 \\
\hline & miscellaneous & Unamed sp. & $\mathrm{F}$ & । & 44 & 52 \\
\hline
\end{tabular}


Fleas

unamed sp.

Lice sp.

Insecta

Cestoda

Macrocyclops albidus

Reptilia

\section{Rhynchocephalia}

Sphenodontidae

Sphenodon punctatus

\section{Squamata}

Anguidae

Eucoccidiorida $\begin{array}{llllll}\text { Arachnida } & \text { Tick sp. } & \text { M } & \text { I } & 44 & 52\end{array}$

M

44

52

\begin{tabular}{|c|c|c|c|c|c|c|}
\hline $\begin{array}{l}\text { Schistocephalus } \\
\text { solidu }\end{array}$ & $M$ & $\mathrm{P}$ & 25 & 157 & Behaviour & $\begin{array}{l}\text { Wedekind \& } \\
\text { Jakobsen } \\
\text { (1998) }\end{array}$ \\
\hline
\end{tabular}

Hepatozoon tuatarae

M I

Social network, host

Godfrey et al. size 
Anguis fragilis

\section{Dactyloidae}

Anolis gundlachi

Lacertidae

Lacerta viridis

Phrynosomatidae

Sceloporus occidentalis

\section{Pythonidae}

Python regius

Scincidae

Eulamprus quoyii
Nematoda

Neoxysomatium

brevicaudatum

Protozoa

Plasmodium sp.

M

P,I 3296

Ixodes ricinus

Plasmodium

mexicanum

Arachnida

Ixodida

Mesostigmata

Geckobiella texana

Ixodes pacificus

Mite

Siphonaptera

Ticks

M

Eucoccidiorida
Hepatozoon hinuliae
Immune system,

male body size

(a)

mondson

(2014)

Schall et al.

(2000a)

conditions

Vaclav et al. (2007)

Schall \& Marghoob

(1995)

Schall et al.

(2000b) geography

Plasma

Testosterone

Cox \& JohnAlder (2007)

Salkeld \& Schwarzkopf (2005) 


\section{Sites and permits}

Zealandia: Thanks to Raewyn Empsom and the team at Zealandia for access to the site for data collection and logistical support when required.

Greater Wellington Regional Council (GWRC): Thank you to Owen Spearpoint, Sara Moylen and the team at GWRC for access to Kaitoke Regional Park and Butterfly Creek for data collection. Thanks also to local Iwi for permission to collect data in the Wellington area.

\section{Funding}

Wellington Botanical Society Research Grant 2014.

- $\quad$ Funding assisted research for Chapter 3.

Victoria University of Wellington Doctoral Scholarship 2012 - 2015.

- $\quad$ All research carried out by KJY was supported by this scholarship. 